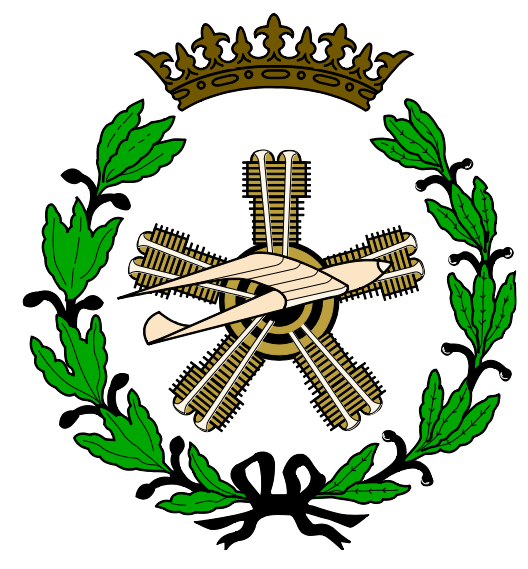

Universidad Politécnica de Madrid

Escuela Técnica Superior de Ingenieros Aeronáuticos

Early management of uncertainty and variability during the design process

Doctoral Thesis

Óscar Lara Rapp

MSc. in Aerospace Engineering 

Departamento de Mecánica de Fluidos y Propulsión Aeroespacial

Escuela Técnica Superior de Ingenieros Aeronáuticos

Early management of uncertainty and variability during the design process

\author{
Óscar Lara Rapp \\ MSc. in Aerospace Engineering \\ Author \\ Efrén Moreno Benavides \\ MSc. in Aerospace Engineering \\ MSc. in Physical Science \\ PhD. in Aerospace Engineering \\ Director
}

2017 
EARLY MANAGEMENT OF UNCERTAINTY AND VARIABILITY DURING THE DESIGN PROCESS 
Tribunal nombrado por el Sr. Rector Magfco. de la Universidad Politécnica de Madrid, el día de de 2017

Presidente:

Vocal:

Vocal:

Vocal:

Secretario:

Suplente:

Suplente:

Realizado el acto de defensa y lectura de la Tesis el día de de 2017 en la E.T.S.I./Facultad

Calificación

EL PRESIDENTE

LOS VOCALES 
EARLY MANAGEMENT OF UNCERTAINTY AND VARIABILITY DURING THE DESIGN PROCESS 


\section{Abstract (English)}

This thesis studies from different perspectives how uncertainty and variability can be managed in the early stages of engineering design processes. Firstly, providing designers with a tool for dealing with this uncertainty and variability in the preliminary stages of the design process and secondly, evaluating how preliminary decision-making tools such as the one presented can impact on the design process and its associated uncertainty and variability.

The thesis is divided into four sections. The aim of the first section is to propose necessary and sufficient conditions for robustness under uncertainty and variability in design scenarios where interval requirements are present. To this end, a set of definitions and a postulate about unpredictable variability and uncertainty are presented, proving that minimizing the number and strength of dependences is a sufficient and necessary condition for robustness. This will characterize the Ideal Output as the utopic target of the design process. A definition of value is introduced and, based on it, the study shows how minimizing the number and strength of dependences increases value. This result lets us present new definitions of needs and constraints. Finally it is proved how Axiomatic Design's Independence and Information Axioms can be derived as theorems in the frame of this work. In addition, it is shown how Axiomatic Design is a necessary but not sufficient condition for robustness.

The second section presents a methodology based on the results of the previous section. The objective of this methodology is to propose a number of steps which help the designer to reduce in a systematic way the number and strength of dependences. This methodology proposes Black Box Items as the basic brick for building solutions, which are items into which a given stimuli impinge and out of which reactions emerge whereas their constitution and structure are 
altogether irrelevant. The usefulness of the methodology is illustrated with a practical case representing the design process of an electrical heater.

The third section of the thesis introduces a set of definitions and properties to characterize the Ideal Design Process and the resources associated to it when variability and uncertainty are present. Both characterizations let determine the impact of preliminary decision-making tools, such as the one presented in the first section, on the consumption of resources and its uncertainty. The section ends deriving the conditions that a preliminary decision-making tool needs to satisfy to improve the design process. It is assumed the design process ameliorates when the best output is obtained with a lower consumption of resources (time and money) and with a lower uncertainty and variability, both concepts related to acquired complexity and risk. Axiomatic Design is studied under this framework, showing evidences that indicate the satisfaction of this set of properties. Design scenarios where the preliminary decision-making tool deteriorates the design process are also found. The last part of this section assesses how the design methodology presented in the second section of the thesis can affect the overall value of the design, understood as the conjunction of the output value plus the process value. The value obtained by this methodology is compared to the one of Axiomatic Design.

The fourth section of the thesis assesses how the results obtained in the previous sections can impact on an organization implementing them and on a real business environment. In this process, key points of improvement and areas of interest in the current business practices are identified for subsequently showing how the results of this work can cope with them. 


\section{Abstract (Español)}

El principal objetivo de la tesis es estudiar desde diferentes perspectivas cómo la incertidumbre y la variabilidad pueden ser gestionadas en las etapas tempranas de los procesos de diseño en ingeniería. Primero, proporcionando a los diseñadores una herramienta para tratar esta incertidumbre y variabilidad durante las etapas preliminares del proceso de diseño y segundo, evaluando cómo las herramientas de toma de decisiones preliminares, tales como la presentada, pueden afectar al proceso de diseño y a la incertidumbre y variabilidad asociadas a éste.

La tesis está dividida en cuatro partes. El objetivo de la primera parte es proponer una serie de condiciones suficientes y necesarias para la robustez en escenarios de diseño donde la incertidumbre y la variabilidad están presentes junto a interval requirements. Para ese fin, se presenta un conjunto de definiciones y un postulado acerca de la incertidumbre y la variabilidad impredecible, probando que el minimizar el número y la fuerza de las dependencias es una condición suficiente y necesaria para la robustez. Este resultado caracterizará el Ideal Output como la meta utópica del proceso de diseño. Así mismo se propone una definición de valor y, basado en ésta, el estudio muestra como la minimización del número y la fuerza de las dependencias aumenta el valor. Este resultado nos permite proporcionar nuevas definiciones de needs y constraints. En la última parte se demuestra cómo los axiomas de independencia y de información del Diseño Axiomático pueden obtenerse como teoremas dentro del marco de este estudio. Finalmente, se prueba cómo el Diseño Axiomático es una condición necesaria pero no suficiente para la robustez.

La segunda parte de la tesis presenta una metodología basada en los resultados de la parte anterior. El objetivo de esta metodología es proponer un número de pasos que puedan ayudar al diseñador a reducir de una forma sistemática el número y la fuerza de las dependencias. Esta metodología propone los Black Box Items como el ladrillo básico que se ha de emplear para 
construir las diferentes soluciones. Estos entes son elementos en los cuales incide un estímulo y de los cuales surgen reacciones, y cuya constitución y estructura es totalmente irrelevante. La utilidad de la metodología se ilustra con un caso práctico que representa el diseño de un calentador eléctrico.

La tercera parte de la tesis introduce un conjunto de definiciones y propiedades con el objetivo de caracterizar el Ideal Design Process y los recursos asociados a éste. Esta caracterización se aplica al estudio del impacto de las herramientas de toma de decisiones preliminares en el consumo de recursos y en la incertidumbre y variabilidad asociadas a éstos, obteniendo finalmente las condiciones que una herramienta de toma de decisiones preliminar ha de presentar para mejorar el proceso de diseño. Se asume que el proceso de diseño mejora cuando el mejor ouptut se obtiene con un menor consumo de recursos (tiempo y dinero) y con una incertidumbre y variabilidad más baja, ambos conceptos asociados a la complejidad adquirida y al riesgo. El Diseño Axiomático se estudia bajo este marco, mostrando evidencias que indican que estas propiedades se satisfacen. Así mismo también se encuentran escenarios de diseño donde las herramientas de toma de decisiones preliminares pueden empeorar el proceso de diseño. Finalmente se evalúa cómo la metodología de diseño presentada en la segunda parte de la tesis puede afectar al valor total del diseño, entendido como la unión del valor del output y el valor del proceso. El valor de esta metodología se comparara con el obtenido por medio del Diseño Axiomático.

La cuarta parte de la tesis estudia el valor que pueden proporcionan los anteriores resultados en una organización dispuesta a implementarlos. En este proceso se identifican los puntos clave de mejora así como diversas áreas de interés en las industrias actuales para, a continuación, mostrar cómo los resultados de este trabajo pueden ayudar en su evaluación y mejora. 


\section{Index}

1.1. OBJECTIVES AND METHODOLOGY

2.1. INTRODUCTION

2.2. STAKeholders

2.4. THE DESIGNeRs' MOdeL

2.5. VARIABILITY AND UNCERTAINTY

2.6.1. THEOREM 1: MAKE THE OUTPUT AS ROBUST AS POSSIBLE

2.6.2. THEOREM 2: MAKE THE ACCEPTANCE INTERVALS AS LARGE AS POSSIBLE

2.6.3. THEOREM 3: MINIMIZE THE NUMBER OF INTERVAL REQUIREMENTS AS MUCH AS POSSIBLE

2.6.4. THEOREM 4: MAKE THE OUTPUT AS LINEAR AS POSSIBLE 
2.6.6. THEOREM 6: MINIMIZE THE NUMBER OF CONDITION REQUIREMENTS AS MUCH AS POSSIBLE

2.6.7. THEOREM 7: MINIMIZE THE NUMBER OF STAKEHOLDERS' REQUIREMENTS AS MUCH AS POSSIBLE

2.6.8. THEOREM 8: MAKE THE OUTPUT AS INSENSITIVE AS POSSIBLE

2.6.9. THEOREM 9: MINIMIZE THE NUMBER AND THE STRENGTH OF THE DEPENDENCES ON UNPREDICTABLE VARIATIONS

2.6.10. THEOREM 10: MINIMIZE THE NUMBER AND THE STRENGTH OF ALL DEPENDENCES AS MUCH AS POSSIBLE 48

2.6.11. THEOREM 11: MINIMIZING THE NUMBER AND STRENGTH OF DEPENDENCES MAKES THE OUTPUT ROBUST 50

2.6.12. THEOREM 12: MINIMIZING THE NUMBER AND STRENGTH OF DEPENDENCES INCREASES THE OUTPUT VALUE 51

2.6.13. THEOREM 13: THE IDEAL OUTPUT

2.7. DisCuSSION ABOUt COROLLARY 3 AND THEOREM 8

2.8. COMmentS ON AxiOMATIC DeSIGN 60

2.9. CONCLUSION

3. A DESIGN METHODOLOGY FOR MINIMIZING DEPENDENCES 67

$\begin{array}{ll}\text { 3.1. INTRODUCTION } & 67\end{array}$

3.2. BLACK BOX ITEMS 68

3.3. DEPENDENCES

3.3.1. STIMULI-REACTIONS DEPENDENCES

3.3.2. INTERNAL DEPENDENCES

3.3.3. COMPLEMENTARY DEPENDENCES

3.4. COSTS AND DEPENDENCES

3.5. Methodology

3.6. EXAMPLE

$\begin{array}{ll}\text { 3.7. CONCLUSIONS } & 100\end{array}$ 
$\begin{array}{ll}\text { 4.1. INTRODUCTION } & 101\end{array}$

$\begin{array}{ll}\text { 4.2. DESIGN PROCESS CHARACTERIZATION } & 102\end{array}$

$\begin{array}{ll}\text { 4.2.1. USEFUL KNOWLEDGE } & 103\end{array}$

$\begin{array}{ll}\text { 4.2.2. IDEAL DESIGN PROCESS } & 104\end{array}$

$\begin{array}{ll}\text { 4.2.3. Phases of the Ideal Design Process } & 107\end{array}$

4.2.4. The StOchastic natURE OF THE IDEAL DESIGN PROCESS 113

$\begin{array}{ll}\text { 4.3. Characterization OF THE RESOURCES OF THE DESIGN PROCESS } & 114\end{array}$

$\begin{array}{ll}\text { 4.3.1. MONEY } & 115\end{array}$

$\begin{array}{ll}\text { 4.3.2. TIME } & 116\end{array}$

$\begin{array}{ll}\text { 4.3.3. RESOURCES PROPERTIES } & 116\end{array}$

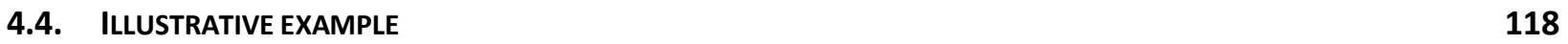

4.5. EVALUATION OF THE IMPACT OF PRELIMINARY DECISION-MAKING TOOLS ON THE CONSUMPTION OF RESOURCES $\begin{array}{ll}\text { AND ITS UNCERTAINTY AND VARIABILITY } & 124\end{array}$

$\begin{array}{ll}\text { 4.5.1. SYNTHESIS-ANALYSIS (SA) DESCRIPTION } & 125\end{array}$

$\begin{array}{lll}\text { 4.5.2. SYNTHESIS-FILTER-ANALYSIS (SFA) DESCRIPTION } & 126\end{array}$

$\begin{array}{ll}\text { 4.5.3. ITERATIONS AND RESOURCES INVOLVED } & 128\end{array}$

$\begin{array}{llr}\text { 4.5.4. Statistical MOdelS } & 130\end{array}$

$\begin{array}{ll}\text { 4.5.4.1. SA configuration } & 130\end{array}$

$\begin{array}{ll}\text { 4.5.4.2. SFA configuration } & 132\end{array}$

4.5.4.3. Relation between design process and design department 135

$\begin{array}{ll}\text { 4.5.5. SA \& SFA COMPARISON } & 135\end{array}$

$\begin{array}{lll}\text { 4.5.5.1. } & \text { Expected time } & 136\end{array}$ 
4.6. LIMITATIONS OF THE PROPOSED APPROACH

4.7. CONCLUSIONS 


\section{List of figures}

Figure 1: Relations between the postulate, definitions and properties

Figure 2: Relations between theorems, corollaries, properties and definitions 50

Figure 3: Black Box Item stimuli and reactions 68

Figure 4: $B B I$ composition example 69

Figure 5: AvO 1 85

Figure 6: AvO 2 88

Figure 7: AvO 3 91

Figure 8: AvO 4 94

Figure 9: Ideal Design Process 1 120

Figure 10: Ideal Design Process 2 121

Figure 11: Design Process 3 123

Figure 12: SA flowchart 125

Figure 13: SFA flowchart 126

Figure 14: Difference between SA and SFA expected time 137

Figure 15: Difference between SA and SFA in terms of variance of time. Cases 1 \& 2 139

Figure 16: Difference between SA and SFA variance of time in case 1 140

Figure 17: Difference between SA and SFA expected expenses. Cases $1 \& 2$ 142

Figure 18: Difference between SA and SFA expected expenses in case 1 143

Figure 19: Difference between SA and SFA variance of expenses. Cases 1 \& 2 144

Figure 20: Difference between SA and SFA variance of time in the case 1 145 
EARLY MANAGEMENT OF UNCERTAINTY AND VARIABILITY DURING THE DESIGN PROCESS 


\section{List of tables}

Table 1: Summary of Stakeholders and their Requirements.

Table 2: Effects of Corollary 3 and Theorem 8 on the dependence between the limits of acceptance under different

sets of design rules 60

Table 3: Cause-effect matrix 79

Table 4: AvO 1 indicators 86

Table 5: Cause-effect matrix of AvO 1 87

Table 6: AvO 2 indicators 89

Table 7: Cause-effect matrix of AvO 2 90

Table 8: AvO 3 indicators 92

Table 9: Cause-effect matrix of AvO 3 93

Table 10: AvO 4 indicators 95

Table 11: Cause-effect matrix of AvO 4 96

Table 12: Power Supply \#SB, \#AS and lowest BBI cost

Table 13: Power supply voltage \#SB, \#AS and the lowest BBI cost 98

Table 14: Resistor \#SB, \#AS and lowest BBI cost 99

Table 15: AvO 1 lowest BBI cost 99

Table 16: Chair AvOs 119

Table 17: Results of Ideal Design Process 1 analysis 121

Table 18: SA sequence 126

Table 19: SFA sequence 128

Table 20: Conditions to be satisfied by the preliminary decision-making tool for improving the design process _ 148

Table 21: Conditions to be satisfied by the preliminary decision-making tool for maximizing the improvement of the design process 149

Table 22: Design process parameters example 150

Table 23: Example of different preliminary decision-making tools 150 
EARLY MANAGEMENT OF UNCERTAINTY AND VARIABILITY DURING THE DESIGN PROCESS

xviii 


\section{List of symbols}

AO Analysis Operator

ASO Advance Synthesis Operator

$\mathrm{AvO} \quad$ Available Outputs

BDP Best Design Process

BO Best Output

C Constraints

CE Conditions Equation

$C_{j} \quad$ Set of AvOs in the iteration number $j$

CR Condition Requirement

D Domain

DCR Designers Condition Requirement

DD Designers Domain

DDI Dimensionless Designers Indicator

$D D M \quad$ Dimensionless Design Matrix

DI Designers Indicator

DIR Designers Interval Requirement

DM Designers' Model

DNI Dimensionless Non-Designers Indicator

DP Design Parameter

$\eta_{z} \quad$ Relative improvement in variable $\mathrm{Z}$

E $\quad$ Expenses 


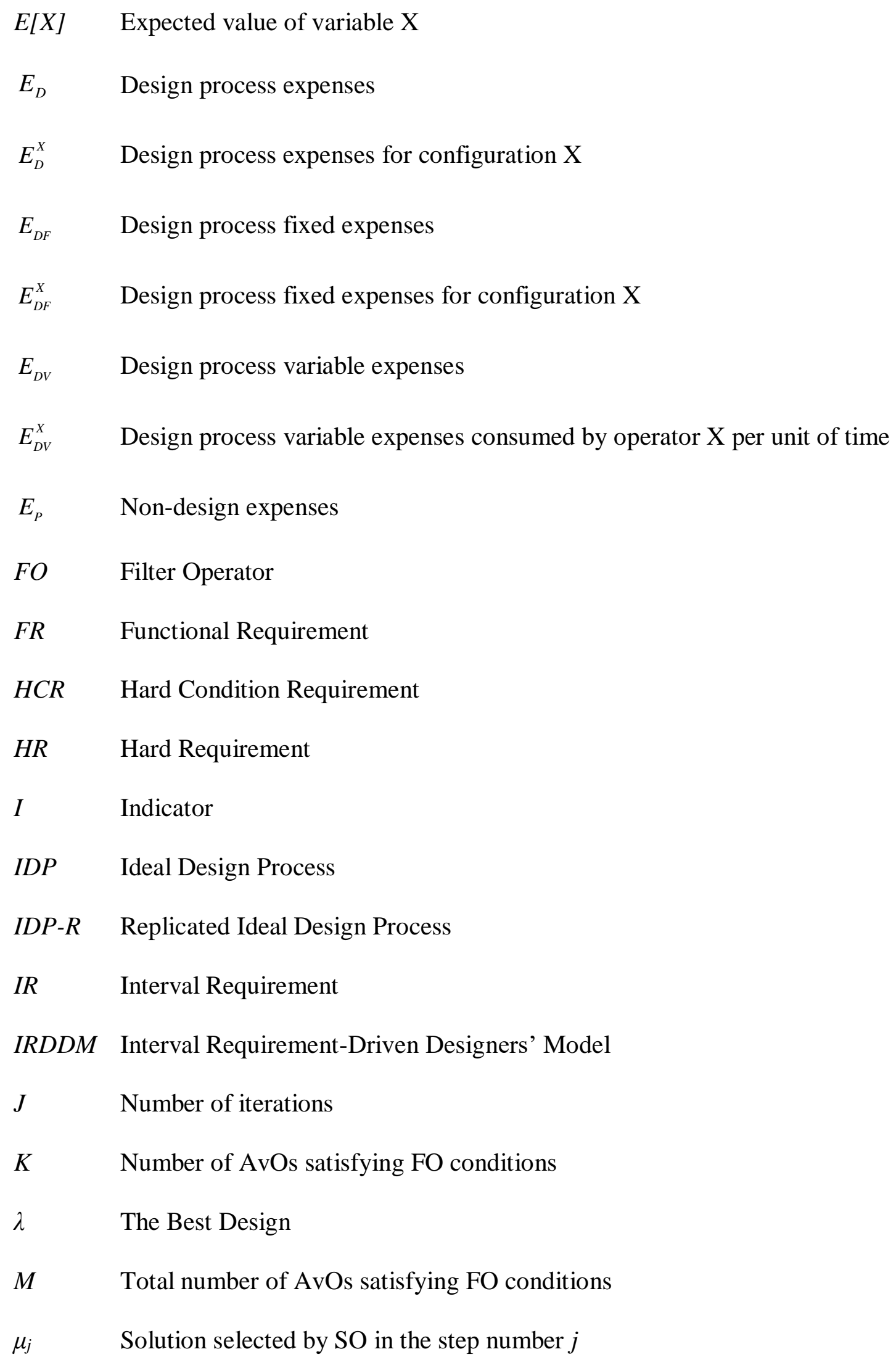




\begin{tabular}{|c|c|}
\hline$N$ & Total Number of AvOs \\
\hline$N C R$ & Non-Designers Condition Requirement \\
\hline$N D$ & Non-Designers Domain \\
\hline$N I$ & Non-Designers Indicator \\
\hline$N I N$ & Net Income \\
\hline$N I R$ & Non-Designers Interval Requirement \\
\hline$\Psi_{k}$ & $k^{\text {th }}$ solution that satisfies FO conditions \\
\hline$R$ & Requirement \\
\hline$R \& D$ & Research and Development \\
\hline$R E$ & Revenues \\
\hline$R M$ & Real Model \\
\hline$S A$ & Synthesis-Analysis Configuration \\
\hline$S C R$ & Soft Condition Requirement \\
\hline$S F A$ & Synthesis-Filter-Analysis Configuration \\
\hline SO & Synthesis Operator \\
\hline$S R$ & Soft Requirement \\
\hline$T$ & Time \\
\hline$T_{D}$ & Design process time \\
\hline$T_{D}^{X}$ & Time required by operator $\mathrm{X}$ per iteration or by configuration $\mathrm{X}$ for the design process \\
\hline $\mathrm{TF}$ & Transfer Function \\
\hline$U D P$ & Utopic Design Process \\
\hline $\operatorname{Var}[X]$ & Variance of variable $\mathrm{X}$ \\
\hline
\end{tabular}

Notation: CR, DCR, DIR, HCR, HR, IR, NCR, NIR, R, SCR and SR represent single elements. 
Their corresponding sets are $\{\mathrm{CR}\},\{\mathrm{DCR}\},\{\mathrm{DIR}\},\{\mathrm{HCR}\},\{\mathrm{HR}\},\{\mathrm{IR}\},\{\mathrm{NCR}\},\{\mathrm{NIR}\},\{\mathrm{R}\}$, $\{\mathrm{SCR}\}$ and $\{\mathrm{SR}\}$. 


\section{INTRODUCTION}

In engineering, the design process of a new product or service is one of the most overriding and challenging tasks an organization has to tackle, always involving some uncertainty about its final success. It is well known (Haik and Shahin 2011) that if a product is not properly designed, no one will be willing to pay for it, no matter how good the manufacturing, production or the rest of the processes related to this product are. The main source for the uncertainty in the success of the design process comes from its definition: it is a process (often iterative), in which the basic sciences, mathematics, and engineering sciences are applied to optimally convert resources to meet a stated objective (Dym 1994, he gives many other definitions of the concept of engineering design process). Hence, the engineering design process comprises a succession of decision-making and creativity problems which involve a huge number of alternatives and criteria. The higher the number of these alternatives and criteria is, the higher the difficulty of the decision is (Kahraman and Çebi 2009) and hence, the higher the uncertainty. Regarding this aspect, Eckert and Clarkson (2010) present an interesting discussion about the uncertainties involved in the design process. Indeed, engineering is not deterministic, as can be suggested by a strict application of formulas, but variability and uncertainty can be found in all engineering decisions (Grayman, 2005). Chiara (2009) explains that uncertainty plays a crucial role both in contemporary science and in much sociological research. This author remarks that uncertainty is often represented as an intrinsic feature of natural and of human phenomenon, citing as examples Heisenberg's uncertainty principle and fuzzy thinking. One aspect to remark here is that uncertainty is an inherent characteristic of the design process regardless of what is being designed: a product, a process or a service. 
This uncertainty is also a consequence of the increasing demands for functionality, cost, quality and sustainability. These requirements are defined by means of complex criteria, leading to a rise in the complexity of the technical systems resulting from the design process (N. P. Suh 2005). Lu and N. P. Suh (2009) explain that the complexity of a system can be divided into inborn complications, due to customer needs and external constraints and acquired complexity, which arises in the design decisions. Avoiding or eliminating acquired complexity during the design phase is the only variable strategy for firms to improve the quality of the resultant design in very competitive markets where the same needs can be satisfied by substitutable products from different companies (Lu and N. P. Suh 2009). Products which have a high number of inborn complications, and hence are complex products, commonly are the result of already lengthy and complicated, and therefore, costly design processes (Ameri, Summers, Mocko, Porter 2008). Thus, this indicates how important it is to reduce as much as possible the acquired complexity in order to keep the overall complexity not higher than strictly needed.

From a financial point of view, Cox (2005) explains by means of figures released from the UK Treasury that the top innovating companies produce $75 \%$ of the revenues from products or services that did not exist five years ago. This is a manifestation of how the investment of resources in the design processes usually gives the highest return for the company. Hence, a big part of the responsibility for obtaining profits and avoiding extra economic losses relies on the design process, which should have an optimum configuration in order to provide the market with the best possible solution for a concrete set of requirements with the minimum resource consumption. As Matthews (2011) states, good design concepts can be transformed into good final products faster than poor design concepts, which usually require greater effort to achieve a similar final quality level. 
Ziv-Av and Reich (2005) explain that the conceptual design stage is the one that dominates almost any product development project when it comes to quality and cost, and thus, the success or failure of the product. The reasons for Cross et al. (1996) why this conceptual stage is so interesting are the following: 1) large amounts of information are processed in a short time, 2) this is the most informal, most complex, least understood and least supported phase by computational tools and 3) decisions and information generated in this phase have a large impact on the rest of the design process and on the overall cost. In addition, changes related to the product can be carried out easier in the early stages of the design process than in the later stages, where modifications can be very expensive in terms of time and money (Haik and Shahin 2011).

The conceptual design process comprises also a succession of decision-making problems. However, these decisions are especially critical since, as Wood and Antonsson (1989) explain, the most important design decisions are made in the preliminary stage. In order to reduce the consumption of extra resources during this stage, it is desirable to have access to preliminary decision-making tools. This idea is also corroborated by Ziv-Av and Reich (2005): it is critical to be able to screen out products that are likely to fail, something that can be achieved by an appropriate preliminary decision-making tool. This, for example, explains why Axiomatic Design is a powerful tool that complements other engineering disciplines during the conceptual design stage (N. P. Suh 1990, 2001; Kulak, Cebi and Kahraman 2010; Benavides 2012). This kind of tools should be a set of criteria such as the Independence and the Information Axioms (N. P. Suh 1990), that allows the designer to rule out the resultant designs which are not the desired one as soon as possible. Benavides (2012) describes how Axiomatic Design can be integrated with other general analysis tools such as entropy, reliability or quality loss function for helping to discard bad solutions as fast as possible. The forte of these tools is that they establish which solutions should 
be analysed in a more detailed way, reducing the resources that otherwise would be invested in analysing solutions which will be discarded during the detailed analysis phase. In other words, as it will be shown in this study, these preliminary tools must be screening tools as powerful, fast and inexpensive as possible in order to reduce the expenses and the time invested in the process.

Since uncertainty plays an important role during the design process, these preliminary decision-making tools should be able to cope with it. For example, Taguchi (1986) says that variability is always present in a design and that designing for having minimum variability is always a goal, what leads him to establish the Quality Loss Function as a fundamental metric (Taguchi, Elsayed and Hsiang [1989], Taguchi [1992] and Wu and Wu [2000]). His message is that deviations from the target value must be avoided, even if the deviation is within tolerance, because all deviations from the target induce unnecessary costs to the society. In line with it, N. P. Suh (1990, 2001), proposed Axiomatic Design theory as a set of design rules derived from two axioms in order to obtain better solutions and reduce this uncertainty.

The main differences between N. P. Suh's (Axiomatic Design) and Taguchi's (Metric Design) approaches come from the qualitative and quantitative nature of their methodologies. $\mathrm{N}$. P. Suh's approach lies on two axioms (Independence and Information Axioms) and two definitions (Functional Requirements and Constraints), which have the purpose of deriving theorems and corollaries for defining the best solution. However, Taguchi's approach lies on postulating that minimum variation is always a need to be satisfied and on the definition of a quadratic form (the Quality Loss Function) that measures the distance of the current output to the best output. Both methodologies have proven to be satisfactory in a large number of situations which include both conceptual and detailed design stages (Osman, Cebi and Kahraman, 2010 and Mori, 2011). However, due to its qualitative nature, Axiomatic Design seems to be more suitable for conceptual 
design, whereas, due to its quantitative nature, Metric Design seems to be more suitable for detailed design. For this reason, Peder (1997) addresses the questions of how and why converting Taguchi's approach into a qualitative tool in order to achieve robustness at the conceptual-design stage. Regarding the 'why', this author explains that the quantitative approach is an aid for inductive reasoning (analysis) about the product, whereas the qualitative approach is an aid for deductive reasoning (synthesis) about the best solution, being the later what largely determines whether the product will be a 'winner' or not. This author states that two different conceptual solutions utilizing different solution principles may feature completely different robustness properties. Regarding the 'how', this author explains that, at concept stage, we are only concerned with the structure and arrangement of elements and the mode of action, whose contributions to the overall robustness of the product may be checked by evaluating concept solutions against a set of general design rules (which are stated in a qualitative way). A study about design rules from the literature is presented by Pahl, Beitz et al. (2007) and a study of the qualitative application of several of these rules is presented by Peder (1997). Axiomatic Design has also shown to be satisfactory to bring up robust designs (El-Haik, 2005 and Mader, 2005).

\subsection{Objectives and methodology}

The objectives of this thesis are divided into four main sections, each addressing different concerns related to uncertainty and variability during the early stages of the design process.

The first section deals with the search for robustness for tackling uncertainty and variability in the product. Despite the variety of approaches presented in the introduction, the literature does not present a statement about when and how a set of design rules ensures output robustness (i.e. what is the sufficient condition for output robustness) but, instead, it shows that there are several necessary ingredients for robustness. Thus, one of the main objectives of this work is to explore 
the theoretical framework which can sustain a set of design principles (qualitative rules) that are both necessary and sufficient conditions for robustness. In this thesis, a theoretical framework can be understood as a set of definitions which lead to a consistent set of theorems that ensures robustness precisely when a set of design rules is satisfied. Therefore, the thesis has to deal with two difficulties which are of different nature. The first difficulty is to introduce a set of definitions which permits to derive the result 'a solution is robust if and only if a set of design requirements is satisfied'. The second difficulty is to derive from this set of definitions the logical argumentations which lead to that statement and to the specific set of design rules which appears in it. Additional concerns addressed by this thesis are if it is possible to establish a notion of output value which may be compatible with this theoretical framework and how different this framework is from N. P. Suh's.

The objective of the second section is to propose a design methodology for minimizing dependences based on the results of the previous section. This design methodology shall implement the previous design rules in a practical way following a coherent and applicable sequence which can be used by designers in real design scenarios where the objective is the minimization of dependences.

The aim of the third section is to study how preliminary decisions making tools can affect the design process and with it, the associated uncertainty and variability. The previous introduction showed how there seems to be a consensus about the necessity of having preliminary decisionmaking tools; however, there is not any scientific literature addressing the impact of this kind of tools as a function of the scenario where the design process is executed. In order to achieve this goal, the first objective of this section is to present a characterization of the Ideal Design Process. This characterization does not try to represent a real design process (that is the reason why the 
“ideal" adjective has been used) but to present an ideal limit of a design process potentially useful for the study of some aspects of the design processes. Following what Reich (1995) explains for the GDT, this study builds a model of the design process with the purpose of fulfilling two additional objectives: the characterization of the resources employed by the design process and the evaluation of the impact of preliminary decision-making tools on the consumption of these resources and its uncertainty and variability. This evaluation is based on how the engineering tools intended for speeding the screening of potential solutions reduce (or increase, if they are not well conceived) the amount of resources consumed, and hence, improve (or deteriorate) the design process. Two different design process configurations that are derived from the Ideal Design Process characterization will be presented for this objective. Eventually, this section studies how the methodology for minimizing dependences proposed in the second section of the thesis affects the overall design value, which is decomposed into the value of the output and the one of the process. The value provided by this methodology is also compared to the value provided by Axiomatic Design

The fourth section of the thesis assess the value that the previous results could provide in a business environment. The objective is to employ the results of this work for tackling current business challenges and areas of improvement and show their potential for dealing with them.

It is important to remark that this thesis must modify some previous definitions and give new ones, although the nomenclature from ISO 9000:2015 has been followed when possible.

\subsection{Structure}

The general structure of this study is divided in four main sections following the previous four-section structure regarding the objectives. 
Section 2 proposes a set of necessary and sufficient conditions for robustness in design scenarios where interval requirements are present. Section 2.1 presents the introduction and Section 2.2 contains the notion of Stakeholders' Requirements and some of their qualifiers: 1) Hard and Soft and 2) Interval and Condition. Section 2.3 classifies Stakeholders into Designers, the ones in charge of satisfying all Requirements, and Non-Designers, which correspond to the rest of Stakeholders. Each of these two sets of Stakeholders has their own Requirements, all of them embedded in the Designers' Model (DM), which contains all the known resultant design dependences and it is presented in Section 2.4. Among all the possible Designers' Models, this study focuses on the ones which have at least one Non-Designers Interval Requirement. In Section 2.5, variability (the effect of chance) and uncertainty (the assessor's lack of knowledge about the parameters which characterize the physical system being modelled) are introduced, discussed and related to previous literature. As a consequence, the notion of unpredictable variability and uncertainty arises allowing us 1) to introduce it as a postulate and 2) to define robustness, as required by the context of this work, at the beginning of Section 2.6. The purpose of Section 2.6 is to derive several theorems and corollaries which set the main characteristics a solution should have in order to satisfy stakeholders' Requirements in the presence of unpredictable uncertainty and variability. This resultant design is called the Ideal Output because it should be the utopic goal for designers who have a scenario similar to the one presented in this work. The main theoretical result is Theorem 10, a Designers' Requirement is to minimize all the dependences, being this the only ingredient required for rebuilding all the previous theorems and corollaries. A notion of output value compatible with the postulate and the definitions of the previous sections is proposed leading to Theorem 13, the Ideal Output maximizes the output value. This discussion permits to give a new definition for the concepts of needs and constraints. Section 2.7 presents a discussion about 
Corollary 3 and Theorem 8 whereas Section 2.8 presents the differences and connections with Axiomatic Design, showing that Axiomatic Design is a particular result of this theoretical framework. Finally Section 2.9 contains the conclusions of this first section.

Section 3 presents a methodology for design scenarios where the minimization of dependences is an objective. Section 3.1 presents an introduction and next section contains the concept of Black Box Items. Section 3.3 classifies dependences and Section 3.4 links them with costs. Next section presents the methodology, which is put into practice in the example of Section 3.6. Finally, Section 3.7 contains the conclusions of this section.

Section 4 focuses on the process rather than on the output. In this way, it presents the Ideal Design Process, which is divided in different subsections. Section 4.1 presents an introduction and Section 4.2 characterizes the Ideal Design Process. Next Section 4.3 does the same with the design process' resources. Section 4.4 presents an illustrative example of the two previous sections. Section 4.5 applies the characterizations of Sections 4.2 and 4.3 to the evaluation of the impact of preliminary decision-making tools on the consumption of resources and its uncertainty and variability. It explains the configurations of the two ideal design processes to be studied and introduces the statistical models which describe them. Both design processes are compared in terms of the resources invested, what permits the derivation of a set of desired characteristics for the preliminary decision-making tool in the last part of Section 4.5 and to link these results with Axiomatic Design and the design methodology presented in Section 3. Section 4.6 discusses the limitations of the proposed approach and Section 4.7 collects the main conclusions of this section.

Finally, Section 5 contains an evaluation of the potential value which could be provided by the results of the thesis in business applications. The thesis finishes with the conclusions in Section 6. 
In addition three appendices are presented. Appendix 1 contains the description of the implicit function theorem (required in Section 2) whereas Appendix 2 presents the projected income statement (required in Section 4) and Appendix 3 explains a particular case of the uniform distribution (required in Section 4). 


\section{IDEAL OUTPUT FOR A ROBUST INTERVAL- DRIVEN DESIGN PROCESS}

\subsection{Introduction}

As explained in the Introduction, the literature lacks a statement about the sufficient condition for output robustness even though it shows how there are several necessary conditions for achieving it. This is a very broad topic which encompasses many different situations and, for this reason, this thesis focuses on design scenarios where interval requirements are present. These scenarios are commonly found in engineering design processes even though they are not the only ones. The objective of this section is to find a set of necessary and sufficient conditions for robustness in design scenarios where interval requirements are present.

In order to reach this objective, a set of definitions is presented which will be complemented with a postulate. This postulate indicates the existence of unpredictable variability and uncertainty in the design process. Thanks to this, a set of theorems and corollaries are obtained which can be eventually condensed in the following statement: minimizing the number and strength of dependences is a sufficient and necessary condition for robustness. This statement will permit the characterization of the Ideal Output as the one which minimizes all dependences, being it a utopic target of the design process. Subsequently, a definition of value is introduced which contains the elements covered by the study. Based on it, it is proven how minimizing the number and strength of dependences increases value, what permits the introduction of new definitions of needs and constraints. 
Eventually, the results obtained in this section are compared to the ones of Axiomatic Design. This comparison shows how Axiomatic Design's Independence and Information Axioms can be derived as theorems in the frame of this work.

\subsection{Stakeholders}

Organizations currently do not only have to respond to customer's interests, but also to others entities: environment (Kobayashi, 2005, Hay, Duffy and Whitfield, 2014), government, society as a whole, etc. Authors like Freeman (2010) or standards like ISO 9000:2015 take this notion into account. For example, the definition of stakeholder given by the standard is 'person or organization that can affect, be affected by, or perceive itself to be affected by a decision or activity'. The same standard defines a process as 'set of interrelated or interacting activities that use inputs to deliver an intended result' and the output as the 'result of a process'. It is remarkable the standard definition of the term design, 'set of processes that transform requirements for an object into more detailed requirements for that object', which will permit splitting the Stakeholders into Designers and Non-Designers in Section 2.3. In this thesis, the process studied is the design and the output is the resultant design, being this anything perceivable or conceivable (an object in terms of the same standard, i.e. a product, service, process, organization or system) which is the result of a set of designer's decisions. A reader not familiar with the standard could replace output by solution, product, resultant design or designed object. Thus, for the purpose of this thesis, Stakeholders are defined as follows:

Definition 1: The Stakeholders of a design (process) are all identifiable groups or individuals who express Requirements (Rs) which affect or can be affected by any activity related to the output and which completely define this output. 
The concept of Requirement follows the definition from ISO 9000:2015, which is 'need or expectation that is stated, generally implied or obligatory'. The inclusion of all the Rs (including the ones needed for defining the output) in the study is aligned with the ISO definition of design. In general, there are three main ingredients which increase the difficulty in the design field and affect the output's suitability for Stakeholders, its robustness and its value: 1) the larger number of Rs coming from an increasing number of Stakeholders, 2) the increasing number of Rs established for longer periods of time and 3) the existence of uncertainty and variability. All these concepts have been widely explored in the existing literature about robustness (see, for example, Kalsi, Hacker and Lewis [1999], Chen and Lewis [1999], Downey, Parkinson and Chase [2003], Durach, Wieland and Machuca [2015] or Wieland and Wallenburg [2012]), and about conceptual design, where N. P. Suh's Axiomatic Design (N. P. Suh, 1990) and Taguchi's Metric Design (Taguchi, 1986) appear as design principles (Benavides, 2012).

Following the notation that will be used along this study, the set of all Rs is denoted as $\{R\}$. As explained in ISO 9000:2015, different qualifiers can be used to denote a specific type of R. In the next lines we will introduce the qualifiers Hard, Soft, Interval, Condition, Designers and Non-Designers. Firstly, the Requirements in Definition 1 can be divided into Hard and Soft Requirements:

Definition 2: Hard Requirements (HRs) are Rs which have to be satisfied for preventing a rejection. Soft Requirements (SRs) are Rs which do not have to be satisfied for preventing a rejection. An output is accepted precisely when all the HRs are satisfied.

Definition 2 divides $\{\mathrm{R}\}$ in such a way that $\{R\}=\{H R\} \cup\{S R\}$. However, since there are different types of Rs, an additional classification can be introduced: 
Definition 3: Interval Requirements (IRs) are Rs which have the following structure: 1) a set of $p \in \mathbb{R}$ Indicators (Is) $\left.\left\{k_{j} \in \mathbb{R} \mid j=1,2, \ldots, p\right\}, 2\right)$ a set of $p \in \mathbb{N}$ intervals defined as $\left\{\left[\underline{k}_{j}, \bar{k}_{j}\right] \subset \mathbb{R} \mid j=1,2, \ldots, p\right\}$, which determine the Domain (D) by the following expression $D=\left[\underline{k}_{1}, \bar{k}_{1}\right] \times\left[\underline{k}_{2}, \bar{k}_{2}\right] \times \ldots \times\left[\underline{k}_{p}, \bar{k}_{p}\right] \subset \mathbb{R}^{p}$ such that a), the length of the intervals is strictly bigger than zero, i.e. $\bar{k}_{i}-\underline{k}_{i}>0(i=1 \ldots p)$ and $\left.b\right)$ the intervals are strictly bounded, i.e. $-\infty<\underline{k}_{i}$ and $\bar{k}_{i}<+\infty(i=1 \ldots p)$, and 3$)$ an acceptance criterion, which sets the acceptance of IRs as $\mathbf{k} \in D$ and the rejection as $\mathbf{k} \notin D$, where $\mathbf{k}=\left(k_{1}, k_{2}, \ldots, k_{p}\right) \in \mathbb{R}^{p}$ is built with the $p$ Is.

Thus, each $\left[\underline{k}_{j}, \bar{k}_{j}\right]$ is an acceptance interval.

This definition of Interval Requirements differs from the definition of Functional Requirements (FRs) provided by Axiomatic Design (N. P. Suh 1990, 2001) because 1) IRs do not have to be independent a priori whereas FRs have to, 2) the set of IRs does not have to be minimum a priori whereas the set of FRs has to, and 3) IRs explicitly include the acceptance intervals whereas the set of FRs does not. This is a significant difference between the two approaches because 1) the independence of the IRs (see Theorem 7), 2) the minimum number of IRs (see Theorem 3) and 3) the introduction of dimensionless variables (see Eq. 12 and 13) will be logical and straightforward consequences of the formulation. These three points will let us treat all the IRs coming from all the different Stakeholders in a uniform dimensionless way (see Section 2.5).

Based on Definition 3, Stakeholders must give the upper and lower admissible values for each Indicator in order to fix the acceptance interval and with it, the maximum allowable variation. For example, if there is an $\mathrm{R}$ demanding 'a shaft whose diameter is equal to $150 \mathrm{~mm}$ with a 
maximum variation of $+/-0.3 \mathrm{~mm}$, this $\mathrm{R}$ is an IR whose Indicator is the diameter and the corresponding acceptance interval is [149.7 $\mathrm{mm}, 150.3 \mathrm{~mm}]$.

Definition 4: Condition Requirements (CRs) are the Rs which are not IRs, such that $\{C R\}=\{C R \in\{R\} / C R \notin\{I R\}\}$. The total number of CRs is $t$.

Definitions 3 and 4 divide Rs into IRs and CRs such that $\{R\}=\{I R\} \cup\{C R\}$. CRs include Rs like 'make constant, make equal to, follow the rule, implement the mathematical law, satisfy, relate, increase, decrease, optimize, maximize, minimize, keep as low as possible, make larger than, etc'. There are situations where the designer has to decide whether an R is an IR or a C. For example, an R for an engine can be written as 1) 'the full-throttle power must be between $225 \mathrm{~kW}$ and $250 \mathrm{~kW}$ ' (IR), and 2) 'increase the full-throttle power as much as possible' (CR).

At this point it is interesting to remark the difference between the acceptance of the resultant design by Stakeholders and the satisfaction that this resultant design brings to them. In this study we follow the definitions of the Oxford Dictionary website as of April $11^{\text {th }} 2016$, where the former is the process or fact of being received as adequate, valid, or suitable whereas the latter is the fulfilment of one's wishes, expectations, or needs, or the pleasure derived from this. Therefore, acceptance is associated with the conformity (as per ISO 9000:2015) of the HRs whereas the satisfaction is associated to the perception of the degree to which the SRs have been fulfilled. This concept of satisfaction is a generalization of the customer satisfaction defined in ISO 9000:2015 with the purpose of including Designers' satisfaction and, in general, all the Stakeholders' satisfaction. Following this discussion, some CRs can be declared as achieved or not achieved in an absolute way (e.g. the car must have five doors). However, there are other CRs which can be achieved only in a shorter or longer extent and hence, increase or decrease the satisfaction in the same extent (e.g. 'this chair has to be as comfortable as possible'). The later CRs 
cannot be said to be completely achieved or not achieved. Hence, different acceptable outputs can bring different degrees of satisfaction and different agreements about how to deal with some SRs (this result will be obtained as Property 5).

Property 1: IRs are always $H R s\{I R\} \subset\{H R\}$.

Proof of Property 1: IRs have an acceptance criterion and hence, if not satisfied, the output is rejected.

Property 1 means that the acceptance criterion of Definition 3 has to be satisfied for satisfying the acceptance criterion of Definition 2.

Property 2: CRs can be Soft Condition Requirements (SCRs) when they belong to SRs or Hard Condition Requirements (HCRs) when they belong to HRs $(\{C R\}=\{H C R\} \cup\{S C R\}$ such that $\{H C R\} \subset\{H R\}$ and $\{S C R\} \subset\{S R\})$.

Proof of Property 2: Since there can be other HRs different from IRs, there can also be CRs which are HRs. Note that Definition 2 does not impose any aspect which forces CRs to be only SRs or HRs.

Property 3: $S C R$ s are the only $S R s(\{S C R\}=\{S R\})$.

Proof of Property 3: As per Definitions 2, 3 and 4 all Rs are divided at the same time into a) SRs and HRs and b) IRs and CRs. Property 1 says that IRs are HRs, so the only remaining Rs which can be SRs are CRs. This set of CRs which are SRs, according to Property 2, is \{SCR\} (i.e. the set containing all SCRs).

Property 4: The following relations are satisfied: 1) $\{R\}=\{I R\} \cup\{H C R\} \cup\{S C R\}$,

2) $\{I R\} \cap\{H C R\}=\varnothing, 3)\{I R\} \cap\{S C R\}=\varnothing$ and 4) $\{H C R\} \cap\{S C R\}=\varnothing$. 
Proof of Property 4: 1) Definition 3 and 4 divide Rs into IRs, and CRs, which as per Property 2 are HCRs and SCRs. 2) IRs, as per Definition 4, cannot be CRs. Since HCRs belong to CRs, this property holds. 3) IRs, as per Definition 4, cannot be CRs. Since SCRs belong to CRs, this property holds. 4) Property 2 divides CRs into SCRs and HCRs. HCRs belong to HRs, which as per Definition 2 are complementary to SRs (with respect to Rs) and hence to SCRs. Thus, their intersection has to be the empty set.

Property 5: If there are two different outputs, Output 1 and Output 2, both accepted (as per Definition 2 criterion), whose respective HRs satisfy $\{H R\}_{1}=\{H R\}_{2}$ (note that by Definition 2 this implies that $\{I R\}_{1}=\{I R\}_{2}$ and $\left.\{H C R\}_{1}=\{H C R\}_{2}\right)$, then $\{S C R\}_{1}=\{S C R\}_{2}$.

Proof of Property 5: If the two outputs are different then they have been defined in a different way so, by Definition 1, their Rs must also be different. If the two outputs are accepted, as per Definition 2, then all their HRs are accepted. As long as these two outputs have the same IRs and HCRs but they are different, they must differ in the remaining set of Rs, which are SCRs as stated by Property 4.

According to Property 5, there can be two solutions for the same problem which lead to a different degree of Stakeholders satisfaction. Hence, the goal should be to establish the SCRs which bring the highest level of satisfaction.

\subsection{Designers and Non-Designers}

As Definition 1 states, Stakeholders set the Rs to be fulfilled by the output. However, depending on their role, this group of Stakeholders can be divided into Designers and NonDesigners. 
Definition 5: Designers are Stakeholders in charge of making the design of an acceptable output (as per Definition 2 acceptance criterion) considering all Rs (as long as Designers are Stakeholders, HRs and SRs include theirs). This subset of Stakeholders is represented by $u$ Designers Condition Requirements (DCRs) such that $\{D C R\} \subset\{C R\}$ and $q$ Designers Interval Requirements (DIRs) such that $\{D I R\} \subset\{I R\}$. Design Indicators (DIs) are represented by $\mathbf{m}=\left(m_{1}, m_{2}, \ldots, m_{q}\right) \in \mathbb{R}^{q}$ and their acceptance intervals determine the Designers Domain (DD) as $D D=\left[\underline{m}_{1}, \bar{m}_{1}\right] \times\left[\underline{m}_{2}, \bar{m}_{2}\right] \times \ldots \times\left[\underline{m}_{q}, \bar{m}_{q}\right] \subset \mathbb{R}^{q}$.

This definition is aligned with the one of Kanukolanu, Lewis and Winer (2004): 'The basic responsibility of a designer is to design a product that meets the requirements of his customer while also satisfying the demands of manufacturing, marketing, etc.' (i.e. satisfying all Rs). Designers' Rs (for example, the components of $\mathbf{m}$ ) are set by Designers and hence, they have to be able to calculate them. If not, they would not set them as Rs. Furthermore, these Rs contain the definition of the product so, if not obtained, the product is not obtained either.

Definition 6: Non-Designers are Stakeholders who are not Designers. This subset of Stakeholders is represented by $v$ Non-Designers Condition Requirements (NCRs) such that $\{N C R\}=\{C R\}-\{D C R\} \subset\{C R\}$ and $r$ Non-Designers Intervals Requirements (NIRs) such that $\{N I R\}=\{I R\}-\{D I R\} \subset\{I R\} . \quad$ Non-Designer Indicators (NIs) are represented by $\mathbf{I}=\left(l_{1}, l_{2}, \ldots, l_{r}\right) \in \mathbb{R}^{r}$ and their acceptance intervals determine Non-Designers Domain (ND) as $N D=\left[\underline{l}_{1}, \bar{l}_{1}\right] \times\left[\underline{l}_{2}, \bar{l}_{2}\right] \times \ldots \times\left[\underline{l}_{r}, \bar{l}_{r}\right] \subset \mathbb{R}^{r}$.

The union of Designers and Non-Designers include all the Stakeholders' Rs. NonDesigners include customers and users but also encompass, for example, the local community surrounding the factory where the product is manufactured, the environment or the carrier in 
charge of transporting and delivering the product. Therefore, although a design process is usually initiated when Designers consider they can design a new solution for satisfying potential customers, the solution to be designed not only affects customers but also other entities like government, environment, organization's shareholders or organization's workers. As Cantamessa, Montagna and Messina (2013) explain, aiming only at buyer's preferences could limit the diffusion of products, besides bounding in general the innovation opportunities. The way how the ND and DD are defined has to take into consideration aspects like the ones explained by Swift et al. (1999) and Smith et al. (1999) regarding the allocation of tolerances. Table 1 summarizes the previous nomenclature.

\begin{tabular}{|c|c|c|c|}
\hline & \multicolumn{2}{|c|}{ Stakeholders } \\
\hline & & Designers & Non-Designers \\
\hline \multirow{4}{*}{ IRs } & Set & DIRs & NIRs \\
\hline & Number & $q$ & $r$ \\
\hline & Indicators & $D I s=\left\{m_{1}, m_{2}, \ldots, m_{q}\right\}$ & $N I s=\left\{l_{1}, l_{2}, \ldots, l_{r}\right\}$ \\
\hline & Domain & $D D=\left[\underline{m}_{1}, \bar{m}_{1}\right] \times \ldots \times\left[\underline{m}_{q}, \bar{m}_{q}\right]$ & $N D=\left[\underline{l}_{1}, \bar{l}_{1}\right] \times \ldots \times\left[\underline{l}_{r}, \bar{l}_{r}\right]$ \\
\hline \multirow{2}{*}{ CRs } & Set & DCRs & NCRs \\
\hline & Number & $u$ & $v$ \\
\hline
\end{tabular}

Table 1: Summary of Stakeholders and their Requirements.

\subsection{The Designers' Model}

This section discusses how the Rs of Designers and Non-Designers are linked to each other.

To this end, the Real Model is defined.

Definition 7: A dependence is each different way of affecting or being affected by an entity. 
This definition of dependence generalizes the definition of Zolghadri, Baron and Girard (2010), where a dependency is defined as a link between modules in a product, by including links between the product and any other entity.

\section{Definition 8: The Real Model (RM) is the description of all the output dependences.}

According to Definition 8, all the dependences of the output with Stakeholders and their Rs are included in the RM. In particular it includes all the dependences known by the designers like correspondences or inequalities. It also contains the laws and relations from the different disciplines (e.g. the laws of physics) which connect DIs to NIs, as well as the equations which implement the required CRs (e.g. the ones for optimizing some functions, which will be a set of partial derivatives equal to zero). Note that every CR must be implemented by means of a RM which should explain how the solution deals with all the CRs at the same time. Therefore, the RM is not the same for a carburettor as for an injection system and it is not the same for an injection system designed to minimize costs as for an injection system designed to maximize the reliability. Thus, different RMs could lead to different degrees of satisfaction. Definition 8 ensures that the RM includes all the dependences and hence it must encompass all the stages of the resultant design life cycle, what indeed follows the idea presented by Stiassnie and Shpitalni (2007) and Mueller and Ganseforth (2012).

It is important to notice that having access to the RM and hence to the description of all the dependences, is an unrealistic situation for Designers. Normally Designers are only able to have access to the description of a certain subset of the set containing all the dependences. For this reason, the Designers' Model is defined as follows:

Definition 9: The Designers' Model (DM) is the description of all the output dependences which are known by Designers. 
It is important to remark that Designers always work with the DM, since this is what they know. The RM is unknown for them except in the case where the DM is equal to the RM (i.e. the DM describes all the output dependences), what entails a full access to all the useful knowledge (it will be described in Definition 19, Section 4.2.1) related to these dependences. However, sometimes this is not enough since there are dependences which might not have been described yet and consequently, they are not yet part of this useful knowledge (e.g. physical laws still to be discovered).

Among the many different possible DMs, the category described in the next definition is going to be the base of the following study.

Definition 10: The Interval Requirement-Driven Designers' Model (IRDDM) is a DM such that the set of NIRs is not empty $(r>0)$ and such that all the NI's values are established by DIs' through at least $r$ real $C^{\infty}$ functions denoted as $\mathbf{D M}_{1}=\mathbf{0}$, where the dimension of $D M_{1}$ must be equal or higher than the number of NIRs, and all the remaining dependences set by CRs are contained in $\mathbf{D M}_{2}$.

Property 6: There is always a DIR in an IRDDM. As a consequence an IRDDM can be expressed as

$$
\begin{gathered}
\mathrm{DM}_{1}(1, \mathrm{~m} ; \mathrm{n})=\mathbf{0} \\
\left(\mathbf{l}, \mathrm{m}, \mathbf{n}, \mathrm{DM}_{2}\right)
\end{gathered}
$$

where $\boldsymbol{n}$ are the set of labels and/or variables required for describing CRs and $\boldsymbol{l}$ and $\boldsymbol{m}$ are the set of indicators described in Table 1.

Proof of Property 6: Definition 10 says that there is at least one NIR in an IRDDM and that NIRs are satisfied by means of DIRs. Hence, there should be at least one DIR. This means that $\mathbf{D M}_{\mathbf{1}}(\mathbf{l}, \mathbf{m} ; \mathbf{n})=\mathbf{0}$ exists as stated in Eq.1. In addition, according to Definition 10, there is 
another part of the IRDDM containing the remaining dependences, $D M_{2}$ (Eq. 2), which is a set of dependences between $\boldsymbol{l}, \boldsymbol{m}$ and $\boldsymbol{n}$.

$\mathrm{DM}_{1}$ and $\mathrm{DM}_{2}$ contain all the known dependences and hence it also includes the CRs related to the set of Rs and the acceptance conditions. For example, $\mathrm{DM}_{2}$ will contain the acceptance conditions in the form of inequalities for each IR: $k_{j} \geq \underline{k}_{j}$ and $k_{j} \leq \bar{k}_{j}$.

Property 7: $D M_{1}$ in an IRDDM can be expressed as a Transfer Function (TF) represented by a function $\mathbf{f}(\mathbf{m} ; \mathbf{n})$ and a Conditions Equation $(C E)$ represented by a function $\mathbf{g}(\mathbf{m} ; \mathbf{n})$. The CE is a set of equations whose dimension has to be equal to $q-r$, being $r$ the dimension of $f$ and $q$ the dimension of $\boldsymbol{m} . \mathrm{DM}_{2}$ in an IRDDM can be expressed as a set of dependences between only variables $\boldsymbol{m}$ and $\boldsymbol{n}$, which is represented by $\boldsymbol{h}$.

$$
\begin{gathered}
\mathbf{f}: \mathbf{m} \rightarrow \mathbf{f}(\mathbf{m} ; \mathbf{n}) \\
\mathbf{g}(\mathbf{m} ; \mathbf{n})=\mathbf{0}
\end{gathered}
$$

$(\mathbf{m}, \mathbf{n}, \mathbf{h})$

Proof of Property 7: According to Definition 10, Designers have to be able to calculate the value of the achieved NIs as a function of the values selected for the DIs (there is at least one DI according to Property 6) when all CRs have been incorporated into the IRDDM. For this reason, $D M_{1}$ should fulfil the premises of the Implicit Function Theorem (see Appendix 1 for a detailed explanation of these premises) so that NIs can be calculated by means of the function given by Eq. 3, whose dimension must be $r$. The rest of the equalities between the variables in the model due to CRs cannot be implemented in the TF and must be considered in a different equation, which is the CE given by $\mathbf{g}^{*}(\mathbf{l}, \mathbf{m} ; \mathbf{n})=\mathbf{0}$. However, according to Eq. 3, $\mathbf{g}^{*}(\mathbf{l}, \mathbf{m} ; \mathbf{n})=\mathbf{g}^{*}(\mathbf{f}(\mathbf{m} ; \mathbf{n}), \mathbf{m} ; \mathbf{n})=\mathbf{g}(\mathbf{m} ; \mathbf{n}), \quad$ which $\quad$ leads $\quad$ to $\quad$ Eq. $4, \quad$ and $\left(\mathbf{f}(\mathbf{m} ; \mathbf{n}), \mathbf{m}, \mathbf{n}, \mathbf{D M}_{2}\right)=(\mathbf{m}, \mathbf{n}, \mathbf{h})$, which leads to Expression 5. A complete model requires 
Designers to calculate all the components of $\boldsymbol{m}$, and hence the Implicit Function Theorem requires the dimension of the $C E$ to be equal to the dimension of $\boldsymbol{m}$ minus the dimension of $\boldsymbol{f}$.

If the number of equalities set by CRs ( $w$ ) is such that $w>q-r$, Designers should remake the DM in order to reduce or transform the excess of CRs (or create more DIs which participate in the equalities). By definition $q+r=p$, with $q \geq 0$ and $r \geq 0$ and $u+v=t$, with $u \geq 0$ and $v \geq 0$. Note that normally $q \gg r$ and $u \gg>v$ holds because the description of the solution requires more parameters than the description of the outcome. Eq. 4 and 5 generalize the concept of constraints employed by E.S. Suh, de Weck and Chang (2007) and Kanukolanu, Lewis and Winer (2004), called in their studies equality and inequality constraints since, in this case, expression 5 not only contains equalities and inequalities but also all other kind of dependences (for example, logical inferences).

\subsection{Variability and uncertainty}

Vose (2000) defines variability (also called aleatory uncertainty by Wynn, Grebici and Clarkson [2011] or stochastic uncertainty by Wood, Antonsson and Beck [1990]) as the effect of chance and hence it is a function of the system (and of the environment and the operation), whereas uncertainty is the assessor's lack of knowledge about the parameters which characterize the physical system being modelled (also called epistemic uncertainty by Wynn, Grebici and Clarkson, 2011). As Goh, McMahon and Booker (2007) explain, there are many different classifications for variability and uncertainty. Goh et al. (2010) also present different classifications for uncertainties and their effects in the costs. All of them can affect the resultant design, firstly at one level, but subsequently at other levels whose effects can stack up (Downey, Parkinson and Chase 2003). For the purpose of this thesis, it is convenient to divide them into unpredictable and predictable. 
Unpredictable refers to the variability and uncertainty which cannot be modelled in advance whereas predictable refers to the one which can be.

- (Unpredictable) uncertainty: The employment of mathematical and physical models which are based on assumptions not reached in the real world (for example, because their parameters and variables cannot be exactly modelled or because the natural laws are not well understood) prevents knowing beforehand the real value of some characteristics and hence, they are sources of uncertainty. For Babuška and Silva (2013), the basic sources of uncertainties coming from data and information are: 1) lack of data with respect to the information, 2) conflicting data with respect to the information, 3) abundance and complexity of information with respect to data, 4) ambiguity of the information and 5) unavailable or not sufficient data and information. This lack of knowledge leads us to state that uncertainty is always unpredictable.

- Unpredictable variability: Software bugs or mistakes resulting from human activities and calculations, processes executions, etc. and unexpected probabilistic distributions of the variables are sources of unpredictable variability. For instance, when the environment where the product is being used is not the expected one because temperature, humidity, electromagnetic interferences, loads and operations levels are different from the ones initially expected during the design process, or when their standard deviations are larger than expected or even when the distribution is completely unknown. Distributions can be partially known due to rounding, measuring, manufacturing processes but hardly completely known (e.g. Srinivasan, Wood and McAdams [1996] or Swift et al. [1999], these last stating that the variability distribution of the manufacturing process is seldom completely known). 
- Predictable variability: Authors like Morrison (1957) already explained fifty years ago that engineering design is based on relations between mean values of the design parameters but, due to variability, each equation should be supplemented by an equation in terms of the variance of the design parameters. Grayman (2005), for example, explains that each piece of data or model should be considered as one observation drawn from a probabilistic distribution.

Chalupnik, Wynn and Clarkson (2013) present an interesting discussion about uncertainty and its impact on different product designs. Wood, Antonsson and Beck (1990) explain that the stochastic uncertainty usually remains during all the design process and Kota and Chakrabarti (2009) that during product development designers often have to face uncertainty. Goh, McMahon and Booker (2007) state that uncertainty in engineering design is inevitable because of its very nature: seeking to find a solution to an abstract and incompletely understood problem. Kota and Chakrabarti (2010) explain that variability and uncertainty can be found during the whole life cycle of the design and Padulo and Guenov (2011) state that the product development process is usually affected by severe uncertainty from the outset. These cases evidence that the presence of unpredictable variability and uncertainty in the design scenarios is very common.

The next postulate sets the design scenario where this study is applicable. It should be noticed that the postulate is a statement which is suggested or assumed as true as the basis for the subsequent reasoning or discussion. Readers should verify whether this postulate suits their particular case or not, although attending to the previous discussion it seems to be applicable to a large number of situations.

Postulate: This study postulates that, due to unpredictable variations (the combination of variability and uncertainty), all the dependences contained in the RM differ from the ones in 
the DM and that the moments of the probability distribution functions of the DIs are bounded but unknown a priori.

The Postulate refers to unpredictable variations and hence, for fulfilling the premise of the Postulate, it is enough for the DM or the probabilistic distribution functions of DIRs to be affected by either unpredictable variability or uncertainty, which are the reasons why the DM differs from the RM. At the same time, the premise of having unknown probability distribution functions is aligned with the characteristic 2.a of Definition 3 which is called hereafter the fluctuation attribute. The premise of being bounded is aligned with the characteristic 2.b provided in that same definition, which is called hereafter the feasibility attribute.

Dealing with these variations is very important as Kazmer and Roser (1999) explain in their robustness discussion with an example stating that small changes in the specification of a wall thickness for a moulded part can lead to big swings in the cavity pressure distribution which can affect the material shrinkage, rendering the product unacceptable. The Postulate represents scenarios where there are factors like those which can be hardly anticipated and cause variations in the resultant designs, not only at a given moment, but also in their evolutions as time flows.

Property 8: The DM differs from the RM in such a way that

$$
\mathbf{R M} \equiv \mathbf{D M} \cup \text { unknown dependences }
$$

Proof of Property 8: The Postulate indicates that the dependences in the RM and the DM differ due to unpredictable variations. As per Definitions 8 and 9, these differences can be only expressed by a set of unknown dependences.

Property 9: The values of DIs differ from the values prescribed by Designers $(\hat{\mathbf{m}})$ in such a way that

$$
\mathbf{m}=\hat{\mathbf{m}}+\boldsymbol{\delta}_{\mathbf{m}}
$$


where $\boldsymbol{\delta}_{\mathrm{m}}$ represents the unknown factor affecting the probability distribution functions of DIs.

Proof of Property 9: The Postulate indicates that unpredictable variations impact on the probability distribution function of DIs. Hence, the values of DIs will differ from the values set by Designers $(\hat{\mathbf{m}})$.

Since Designers know the DM (not the RM), the TF, CE and $\mathrm{DM}_{2}$ will come from the DM (not from the RM) and consequently NIs and NCRs are affected by unpredictable variations which are not included in the model. This means that NIs have two different sources of variations, firstly the variations due to the unknown probability distribution function of DIs and secondly variations coming from the unknown dependences. These unknown dependences of Eq. 6 causing the variations in the RM and induced by the Postulate, are going to be modelled using a set of deltas: $\boldsymbol{\delta}_{\mathbf{f}}, \boldsymbol{\delta}_{\mathrm{g}}$ and $\boldsymbol{\delta}_{\mathrm{h}}$. Note that in general these deltas might depend (or not) on the values of $\mathbf{m}$ and $\mathbf{n}$; although this fact is not relevant for the argumentation, this is expressed explicitly. When these unknown variations are added to the DM, NIs are obtained in the following way:

$$
\begin{gathered}
\mathbf{l}=\mathbf{f}(\mathbf{m} ; \mathbf{n})+\boldsymbol{\delta}_{\mathbf{f}}(\mathbf{m} ; \mathbf{n}) \\
\mathbf{g}(\mathbf{m} ; \mathbf{n})+\boldsymbol{\delta}_{\mathbf{g}}(\mathbf{m} ; \mathbf{n})=\mathbf{0} \\
\left(\mathbf{m}, \mathbf{n}, \mathbf{h} \cup \boldsymbol{\delta}_{\mathbf{h}}\right) \\
\mathbf{m}=\hat{\mathbf{m}}+\boldsymbol{\delta}_{\mathbf{m}}
\end{gathered}
$$

Property 10: An acceptable output entails a) $\mathbf{l} \in N D, \quad$ b) $\mathbf{m} \in D D$ and c) $\mathbf{f}(D D)+\boldsymbol{\delta}_{\mathbf{f}}(D D) \subseteq N D$

Proof of Property 10: The acceptance criterion from Definition 3 requires Is to stay inside D, i.e. $\mathbf{k} \in D$, where $\mathbf{k}$, attending to Table 1 , is denoted as $\boldsymbol{l}$ if it represents NIs or as $\boldsymbol{m}$ if it represents DIs, as Definitions 5 and 6 require. Hence, the acceptance criterion can be 
expressed as the conjunction of $\mathbf{m} \in D D$ and $\mathbf{l} \in N D$, which as per Eq. 8 entail $\mathbf{f}(D D)+\boldsymbol{\delta}_{\mathbf{f}}(D D) \subseteq N D$.

The structure of the domains in Definitions 3,5 and 6 suggests the changes of variables which convert NIs and DIs into the following dimensionless parameters: 1) Dimensionless NonDesigners Indicators (DNIs) $\mathbf{y}=\left(y_{1}, y_{2}, \ldots, y_{r}\right) \in \mathbb{R}^{r}$ and 2) Dimensionless Designers Indicators (DDIs) $\mathbf{x}=\left(x_{1}, x_{2}, \ldots, x_{q}\right) \in \mathbb{R}^{q}$. This codification is defined as (Benavides 2012)

$$
\begin{gathered}
x_{j}=\frac{m_{j}-\frac{\bar{m}_{j}+\underline{m}_{j}}{2}}{\frac{\bar{m}_{j}-\underline{m}_{j}}{2}} ; j=1,2, \ldots, q \\
y_{i}=\frac{\bar{l}_{i}-\frac{\bar{l}_{i}+\underline{l}_{i}}{2}}{\frac{\bar{l}_{i}-\underline{l}_{i}}{2}} ; i=1,2, \ldots, r
\end{gathered}
$$

Fluctuation and feasibility attributes ensure that these codifications are well defined. As a result, hyper-volumes ND and DD are transformed respectively into the Dimensionless NonDesigners Domain $\left([-1,1]^{r}\right)$ and Dimensionless Designers Domain $\left([-1,1]^{q}\right)$ :

$$
\begin{aligned}
& {[-1,1]^{q}=[-1,1] \times[-1,1] \times \ldots \times[-1,1] \subset \mathbb{R}^{q}} \\
& {[-1,1]^{r}=[-1,1] \times[-1,1] \times \ldots \times[-1,1] \subset \mathbb{R}^{r}}
\end{aligned}
$$

In this codification DNIs and DDIs have the same acceptance interval: $[-1,1]$. The only difference is the dimension of their spaces, which is $r$ in one case and $q$ in the other. In order to express the RM in the new variables, the following nomenclature is defined:

$$
\begin{gathered}
\mathbf{m}_{\mathbf{o}}=\left(\frac{\bar{m}_{1}+\underline{m}_{1}}{2}, \frac{\bar{m}_{2}+\underline{m}_{2}}{2}, \ldots, \frac{\bar{m}_{q}+\underline{m}_{q}}{2}\right) \in \mathbb{R}^{q} \\
\mathbf{I}_{\mathbf{o}}=\left(\frac{\bar{l}_{1}+\underline{l}_{1}}{2}, \frac{\bar{l}_{2}+\underline{l}_{2}}{2}, \ldots, \frac{\bar{l}_{r}+\underline{l}_{r}}{2}\right) \in \mathbb{R}^{r}
\end{gathered}
$$


Let us also define the matrices $\mathbf{M} \in \mathbb{M}(q, q)$ and $\mathbf{L} \in \mathbb{M}(r, r)$ such that

$$
\begin{gathered}
M_{i j}=\left\{\begin{array}{c}
0 \text { if } i \neq j \\
\frac{\bar{m}_{j}-\underline{m}_{j}}{2} \text { if } i=j
\end{array}\right. \\
L_{i j}=\left\{\begin{array}{c}
0 \text { if } i \neq j \\
\frac{\bar{l}_{i}-\underline{l}_{i}}{2} \text { if } i=j
\end{array}\right.
\end{gathered}
$$

Note that, due to fluctuation and feasibility attributes, $\mathbf{M}$ and $\mathbf{L}$ are well-defined and invertible. This allows us to write the expressions 8 to 11 as

$$
\begin{gathered}
\mathbf{y}(\mathbf{x})=\mathbf{L}^{-1}\left[\mathbf{f}\left(\mathbf{M x}+\mathbf{m}_{0} ; \mathbf{n}\right)-\mathbf{l}_{\mathbf{o}}\right]+\mathbf{V}(\mathbf{x} ; \mathbf{n}) \\
\mathbf{V}(\mathbf{x} ; \mathbf{n})=\mathbf{L}^{-1} \boldsymbol{\delta}_{\mathbf{f}}\left(\mathbf{M x}+\mathbf{m}_{0} ; \mathbf{n}\right) \\
\mathbf{g}\left(\mathbf{M x}+\mathbf{m}_{0} ; \mathbf{n}\right)+\boldsymbol{\delta}_{\mathbf{g}}\left(\mathbf{M x}+\mathbf{m}_{\mathbf{0}} ; \mathbf{n}\right)=\mathbf{0} \\
\left(\mathbf{M x}+\mathbf{m}_{0}, \mathbf{n}, \mathbf{h} \cup \boldsymbol{\delta}_{\mathbf{h}}\right) \\
\mathbf{x}=\mathbf{M}^{-1}\left[\mathbf{m}-\mathbf{m}_{\mathbf{0}}\right]=\mathbf{M}^{-1}\left[\widehat{\mathbf{m}}+\boldsymbol{\delta}_{\mathbf{m}}-\mathbf{m}_{\mathbf{0}}\right]
\end{gathered}
$$

In these expressions, $\mathbf{V}(\mathbf{x} ; \mathbf{n})$ contains the unpredictable variations in the $\mathrm{TF}$.

Property 11: An acceptable output implies 1) $\mathbf{y} \in[-1,1]^{r}$, 2) $\mathbf{x} \in[-1,1]^{q}$ and 3) $\mathbf{y}\left([-1,1]^{q}\right) \subseteq[-1,1]^{r}$

Proof of Property 11: It is a direct consequence of Property 10 and expressions 12 to 24.

Property 12: An acceptable output implies $-1 \leq y_{i} \leq 1$ for all DNIs.

Proof of Property 12: Since $\mathbf{y} \in[-1,1]^{r}$ as per Property 11.1, each $y_{i}$ has to belong to $[-1,1]$.

Property 13: There always exists at least one dependence linking one NI with the DIs.

Proof of Property 13: If all dependences between one NI and the DIs were removed, there would not be a cause-effect relation between them and this would contradict Definition 10. 
Figure 1 shows the relations between the definitions and the properties. Each box has influences from the previous boxes to which it is connected. Note that Property 13 is an important result because it establishes that the set of dependences (and hence the DM) is never an empty set even though the results of the next section will tend systematically to reduce the number of dependences to zero (see for example Corollary 9). From this, the following question arises: what is the minimum number of dependences a design process could achieve? The following corollaries and theorems address, among others, this important question.

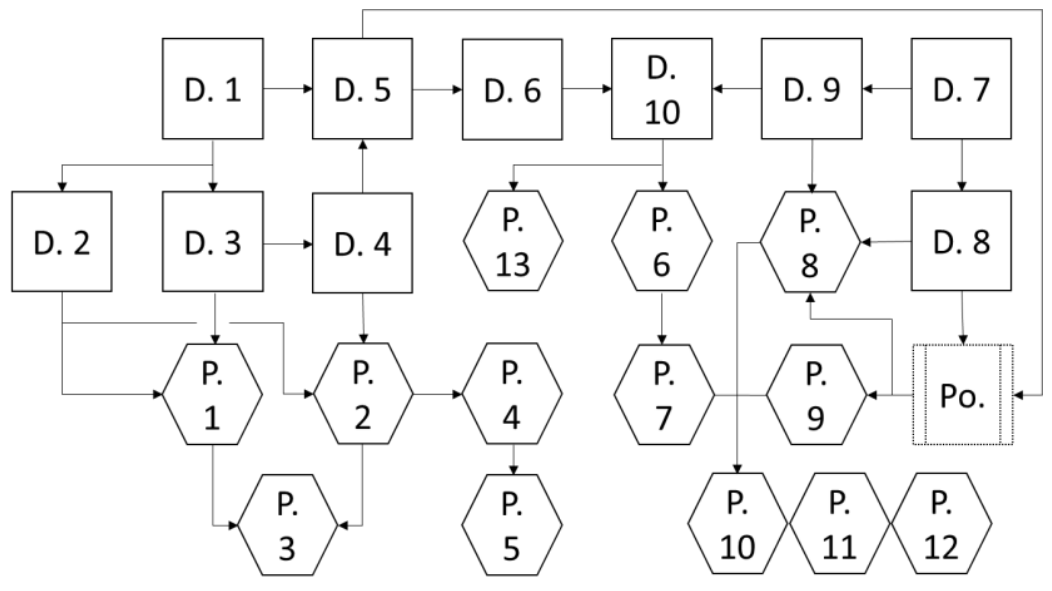

Figure 1: Relations between the postulate, definitions and properties

\subsection{The Ideal Output}

\subsubsection{Theorem 1: Make the output as robust as possible}

Based on the definitions of robustness provided by Andersson (1997), Downey, Parkinson and Chase (2003), Fowlkes and Creveling (1995), Asbjørnslett and Rausand (1999), Meepetchdee and Shah (2007) and Vlajic and van der Vorst and Haijema (2012), and adapted for the purpose of this study, the following definition is proposed: 
Definition 11: Robustness is the characteristic that an output has of satisfying the acceptance criterion (according to Definition 2) in the presence of unpredictable variations (according to the Postulate). An output which satisfies it is said to be a robust output.

Theorem 1: A Requirement of Designers is to have a robust output.

Proof of Theorem 1: Attending to Definition 5, a Requirement of Designers is to obtain an output which is accepted following the acceptance criterion of Definition 2. However, attending to the Postulate, in this design scenario there always exists unpredictable variations affecting the output. Thus, the output has to satisfy the acceptance criterion in the presence of unpredictable variations, which is a robust output according to Definition 11.

This is not a new result, neither next Theorems 2 and 3. For example Taguchi, Elsayed and Hsiang (1989) already stated them as a result of minimizing the Quality Loss Function. In this thesis, they are necessary ingredients for the consistency of the following argumentation and are proven in a different way.

\subsubsection{Theorem 2: Make the acceptance intervals as large as possible}

Corollary 1: A Requirement of Designers is to make $\mathbf{V}(\mathbf{x} ; \mathbf{n}) \rightarrow \mathbf{0}$ and $\mathbf{M}^{-1} \boldsymbol{\delta}_{\mathbf{m}} \rightarrow \mathbf{0}$.

Proof of Corollary 1: As per Property 11, which stems from the acceptance criterion of Definition 3, an acceptable output implies that NIs and DIs have to stay within NIRs' and DIRs' acceptance intervals respectively. However $\mathbf{V}(\mathbf{x} ; \mathbf{n})$ and $\mathbf{M}^{-1} \boldsymbol{\delta}_{\mathbf{m}}$ represent unpredictable variations in Eq. 20 and 24 and they can make DNIs and DDIs fall outside their acceptance intervals. Hence, in order to maximize the acceptance probability as per Property 11 acceptance criteria and Theorem $1, \mathbf{V}(\mathbf{x} ; \mathbf{n})$ and $\mathbf{M}^{-1} \boldsymbol{\delta}_{\mathbf{m}}$ must tend to zero $\left(\mathbf{V}(\mathbf{x} ; \mathbf{n}) \rightarrow \mathbf{0}\right.$ and $\left.\mathbf{M}^{-1} \boldsymbol{\delta}_{\mathbf{m}} \rightarrow \mathbf{0}\right)$ 
Corollary 2: A Requirement of Designers is to make $\bar{l}_{i}-\underline{l}_{i} \rightarrow \infty$.

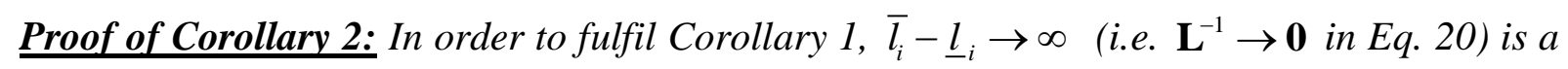
key element for obtaining $\mathbf{V}(\mathbf{x} ; \mathbf{n}) \rightarrow \mathbf{0}$ for any arbitrary value of $\mathbf{V}(\mathbf{x} ; \mathbf{n})$.

There are many situations where Designers cannot modify NIRs. In these situations Corollary 2 should be understood as 'prevent narrowing the acceptance intervals of NIRs unnecessarily and, if possible, widen them'.

Corollary 3: A Requirement of Designers is to make $\bar{m}_{i}-\underline{m}_{i} \rightarrow \infty$.

Proof of Corollary 3: In order to fulfil Corollary $1, \bar{m}_{i}-\underline{m}_{i} \rightarrow \infty$ is a key element for obtaining $\mathbf{M}^{-1} \boldsymbol{\delta}_{\mathbf{m}} \rightarrow \mathbf{0}$ in Eq. 24 for any arbitrary value of $\boldsymbol{\delta}_{\mathbf{m}}$.

Corollary 3 is aligned with Kalsi, Hacker and Lewis (1999), where they state that Designers are much more likely to be able to find an output that satisfies the Rs if they have flexibility for selecting from a range of possible values as opposed to a point solution.

Corollary 4: A Requirement of Designers is to make $\mathbf{m}=\mathbf{m}_{\mathbf{0}}$.

Proof of Corollary 4: As long as variations are unpredictable in Eq. 24, we cannot assume any preferable sign or value for them. For that reason the point $\mathbf{m}=\mathbf{m}_{\mathbf{0}}$, equidistant from the forbidden points $\mathbf{m}=\overline{\mathbf{m}}$ and $\mathbf{m}=\underline{\mathbf{m}}$ (since they do not allow arbitrary variations), is the option which can stand the largest unpredictable variations (Theorem 1).

Theorem 2: A Requirement of Designers is to make all the IRs' acceptance intervals as wide as possible.

Proof of Theorem 2: Corollaries 2 and 3 prove that Designers' and Non-Designers' acceptance intervals should be as wide as possible. Since Definitions 5 and 6 state that they are all the acceptance intervals, Theorem 2 holds. 
Corollary 5: A Requirement of Designers is to make all the tolerances as large as possible.

Proof of Corollary 5: It is a direct consequence of Theorem 2 because tolerances are Designer's acceptance intervals.

A similar conclusion to the one of Corollary 5 is also reported by N.P. Suh (1990) and Taguchi, Elsayed and Hsiang (1989).

Corollary 6: A Requirement of Designers is to convert all the IRs into CRs.

Proof of Corollary 6: Theorem 2 (i.e. $\bar{l}_{i}-\underline{l}_{i} \rightarrow \infty$ and $\left.\bar{m}_{i}-\underline{m}_{i} \rightarrow \infty\right)$ tend to the cases $(-\infty,+\infty)$ , $(-\infty, \bar{k})$ or $(\underline{k},+\infty)$, which by Definition 3 are not IRs because they do not satisfy the feasibility attribute and hence, by Definition 4, they must be CRs (note that the first one is the CR of removing the $R$ ).

\subsubsection{Theorem 3: Minimize the number of Interval Requirements as much as possible}

Corollary 7: A Requirement of Designers is to reduce the number of NIRs.

Proof of Corollary 7: Given one NIR, Corollary 6 converts it into a CR. As a consequence, the number of NIRs is reduced.

Corollary 8: A Requirement of Designers is to reduce the number of DIRs.

Proof of Corollary 8: Given one DIR, Corollary 6 converts it into a CR. As a consequence, the number of DIRs is reduced.

Alternate proof of Corollary 8: Taking into account that $f$ is restricted to real $C^{\infty}$ functions (Definition 10), the Taylor expansion of Eq. 20 around $\boldsymbol{x}=\boldsymbol{0}$ (see Corollary 4) is

$$
\mathbf{y}(\mathbf{x})=\mathbf{L}^{-1}\left[\mathbf{f}\left(\mathbf{M x}+\mathbf{m}_{0} ; \mathbf{n}\right)-\mathbf{l}_{\mathbf{o}}\right]=
$$




$$
\mathbf{a}+\sum_{j=1}^{q} \mathbf{a}_{j} x_{j}+\sum_{k=1}^{q} \sum_{j=1}^{q} \mathbf{a}_{j k} x_{j} x_{k}+\sum_{l=1}^{q} \sum_{k=1}^{q} \sum_{j=1}^{q} \mathbf{a}_{j k l} x_{j} x_{k} x_{l}+\ldots+\mathbf{V}(\mathbf{x} ; \mathbf{n})
$$

The mean of $\boldsymbol{y}$, a key value for Designers, is calculated as

$$
E[\mathbf{y}]=\mathbf{a}+\sum_{j=1}^{q} \mathbf{a}_{j} E\left[x_{j}\right]+\sum_{k=1}^{q} \sum_{j=1}^{q} \mathbf{a}_{j k} E\left[x_{j} x_{k}\right]+\sum_{l=1}^{q} \sum_{k=1}^{q} \sum_{j=1}^{q} \mathbf{a}_{j k l} E\left[x_{j} x_{k} x_{l}\right]+\ldots+E[\mathbf{V}(\mathbf{x} ; \mathbf{n})]
$$

Since the number of DIRs is $q>0$ (Property 6), the $n^{\text {th }}$ term in the expansion involves $\bar{C}_{n}^{q}=C_{n}^{q+n-1}=\left(\begin{array}{c}q+n-1 \\ n\end{array}\right)=\frac{(q+n-1) !}{n !(q-1) !} \quad$ different statistical moments (combinations with repetition) for the component $y_{i}$ which are unknown to Designers (Postulate). If the number of DIRs is increased by one, the new number would be $\left(\begin{array}{c}q+n \\ n\end{array}\right)=\frac{(q+n) !}{n ! q !}$, what increases the number of moments because $\left(\begin{array}{c}q+n \\ n\end{array}\right) /\left(\begin{array}{c}q+n-1 \\ n\end{array}\right)=1+n / q>1$ and it makes higher the number of sources of unpredictable variations for the case $q+1$ than for $q$. Thus, a Requirement of Designers is to keep $q$ as low as possible in order to have the minimum level of sources of unpredictable variations during the design process (Theorem 1).

Theorem 3: A Requirement of Designers is to minimize the number of IRs.

Proof of Theorem 3: Corollaries 7 and 8 minimize the number of NIRs and DIRs. By Definitions 5 and 6, NIRs and DIRs are all the IRs. Hence Corollaries 7 and 8 minimize the total number of IRs.

Note that Corollary 8 could lead to a situation where the number of DIRs affecting one NIR is increased if, as a result of this decision, the number of DIRs which affect another NIR is reduced in such a way that the total number of DIRs is reduced. Next corollary shows that this 
situation is not a Requirement of Designers because both sets of IRs should be reduced at the same time.

Corollary 9: A Requirement of Designers is to keep the number of dependences linking one NIR to the different DIRs as low as possible. (This corollary will be a particular case of Theorem 7).

Proof of Corollary 9: The number of different statistical moments affecting $y_{i}$ in the $n^{\text {th }}$ term of Eq. 26 is proportional to the number of non-null coefficients in that term. The Postulate ensures that all these moments are unknown, therefore the number of unpredictable variations sources is larger when the number of non-null coefficients increases. Hence, Designers have the Requirement of minimizing the number of these non-null coefficients in order to minimize the impact of unpredictable variations on the output (Theorem 1). This result is valid for every component in Eq. 26, and hence, it minimizes the number of dependences linking one NI to the different DIs.

\subsubsection{Theorem 4: Make the output as linear as possible}

Theorem 4: A Requirement of Designers is to make the solution as linear as possible.

Proof of Theorem 4: Note that by Definition 10, r cannot be zero. For the case $q>1$, the term $n$ in Eq. 26 involves $\left(\begin{array}{c}q+n-1 \\ n\end{array}\right)=\frac{(q+n-1) !}{n !(q-1) !}$ different statistical moments and the term $n+1$ involves $\left(\begin{array}{c}q+n \\ n+1\end{array}\right)=\frac{(q+n) !}{(n+1) !(\mathrm{q}-1) !}$. Since the number of unknown moments has increased because $\left(\begin{array}{c}q+n \\ n+1\end{array}\right) /\left(\begin{array}{c}q+n-1 \\ n\end{array}\right)=\frac{n+q}{n+1}>1$, the situation with the lowest number of sources of unpredictable variations is the one with the lowest value of $n$, which is $n=1$ (the linear one) 
because Definition 10 and Property 6 prevents $n=0$. For the case $q=1$, the linear term is also preferred according to Theorem 1 because the first moment (the mean) does not depend on the value of other moments whereas the second moment depends on the value of the mean and on the standard deviation $\left(E\left[x^{2}\right]=E\left[(x-E[x])^{2}\right]+E[x]^{2}\right)$ and hence retaining the second term would introduce the unpredictable variations associated to not knowing neither the mean nor the standard deviation. The same happens with the rest of the moments.

In matrix notation, Theorem 4 simplifies Eq. 20 into

$$
\mathbf{y}(\mathbf{x})=\mathbf{a}+\mathbf{A}(\mathbf{n}) \mathbf{x}+\mathbf{V}(\mathbf{x} ; \mathbf{n})
$$

A is the following rectangular matrix of size $r \times q$, called Dimensionless Design Matrix (DDM):

$$
\begin{gathered}
\mathbf{A}(\mathbf{n})=\left(\begin{array}{ccc}
A_{11}(\mathbf{n}) & \ldots & A_{1 q}(\mathbf{n}) \\
\vdots & \ddots & \vdots \\
A_{r 1}(\mathbf{n}) & \ldots & A_{r q}(\mathbf{n})
\end{array}\right) \\
A_{i j}(\mathbf{n})=\frac{\bar{m}_{j}-\underline{m}_{j}}{\bar{l}_{i}-\underline{l}_{i}} \frac{\partial f_{i}\left(m_{0} ; \mathbf{n}\right)}{\partial m_{j}}
\end{gathered}
$$

This form of the design matrix also appears in Benavides (2012). Note that the method for obtaining the DDM is universal because all IRs can be expressed as dimensionless variables (using Eq. 12 and 13) and all the acceptance intervals can be expressed as [-1, 1].

\subsubsection{Theorem 5: Make the Dimensionless Design Matrix regular and as diagonal as possible}

Corollary 10: A Requirement of Designers is a DDM whose rank is equal to r. (Alternate statement: the rank of the DDM should be maximum). 
Proof of Corollary 10: According to Property 13, there always has to be a cause-effect relation between DIs and NIs. Thus, if Theorem 4 is satisfied, the DDM is the only controllable causeeffect relation during the design process (the other is $\mathbf{V}(\mathbf{x} ; \mathbf{n})$ but, since it is unknown, Designers cannot control it). If the DDM rank is lower than $r$, the image of $\mathbb{R}^{q}$ is a subspace of $\mathbb{R}^{r}$ and hence, at least one NIR is a linearly dependent variable with respect to the rest of DIRs. This means the value of this NIR is fixed by the rest of NIRs and consequently, except for some particular cases, all NIRs cannot be satisfied at the same time, contradicting Property 11 criteria.

Corollary 11: A Requirement of Designers is to have a square DDM.

Proof of Corollary 11: Corollary 10 ensures that $q$ has to be bigger or equal to $r$; however, if $q$ is larger than $r$ then there is at least one NIR which has more dependences with DIRs than the minimum required by Property 13, what contradicts Corollary 9.

Theorem 5: A Requirement of Designers is to keep the DDM regular and as diagonal as possible.

Proof of Theorem 5: Corollary 11 makes the matrix square and Corollary 10 full-rank, hence regular and invertible. In addition, Corollary 9 reduces to one the number of DIRs which affect each NIR. This means that the DDM has one and only one non-zero element per row, but as long as the matrix is full-rank, the DDM has one and only one non-zero element per column. Consequently and without any loss of generality because it is always possible to permute the order of rows and columns, the DDM can be written as a diagonal matrix whose determinant is not zero.

Theorem 5 establishes a (square) diagonal matrix as the target, but this is just a mnemonic rule since the relevant fact is that the matrix has to have one and only one non-zero element per 
row and column. It is also interesting to note that this is aligned with what Andersson (1997) stated in the principle of the division of tasks, where he said that robustness is increased if certain functions are assigned to independent elements in a system.

Corollary 12: A Requirement of Designers is to keep the number of DIRs equal to the number of NIRs.

Proof of Corollary 12: If there were more DIRs than NIRs the matrix would not be diagonal and hence Theorem 5 would not be satisfied.

Theorem 5 and Corollary 12 are also classical results of Axiomatic Design (N. P. Suh 1990, 2001), which establishes that the best output has a diagonal square design matrix. However, the design matrix used by N. P. Suh differs from the one used in this proof, which is dimensionless. In addition, taking into account that the starting point of Axiomatic Design and the one presented in this work are different, it is quite interesting that both approaches share this result. This fact is further discussed in Section 2.8.

\subsubsection{Theorem 6: Minimize the number of Condition Requirements as much as possible}

Theorem 6: A Requirement of Designers is to minimize the number and strength of the dependences contained in the Conditions Equation (Eq. 22) and in DM2 (expression 23) what entails minimizing the number of CRs.

Proof of Theorem 6: Eq. 22 and expression 23 represent the CE and the DM2, containing all CRs. These equations are impacted by unpredictable variations through $\boldsymbol{\delta}_{\mathbf{g}}\left(\mathbf{M x}+\mathbf{m}_{\mathbf{0}} ; \mathbf{n}\right)$ and $\boldsymbol{\delta}_{\mathbf{h}}\left(\mathbf{M x}+\mathbf{m}_{\mathbf{0}}\right)$. The lower the number of CRs, the lower the dimension of these equations and expressions and hence the lower the dependences contained in the RM which, by the Postulate, 
are all affected by unpredictable variations. In addition, through the CE and DM2, these variations also affect and are affected by the TF, which subsequently affect NIRs. Hence, there is a set of dependences between the unpredictable variations of the CE and DM2 and NIRs what leads to state that the fewer the number of dependences in CE and DM 2 (and thus, CRs) and their strengths, the lower the impact of unpredictable variations on NIRs, which, as per Theorem 1, is a Designer's Requirement. Deleting a CR removes its variations with respect to the output. Hence, minimizing the number of CRs is also a Requirement.

Note that there should be at least a set of acceptance conditions related to HRs. For example, for each IR, there should be at least 2 inequalities (CRs) contained in the CE ( $k_{j} \geq \underline{k}_{j}$ and $\left.k_{j} \leq \bar{k}_{j}\right)$.

\subsubsection{Theorem 7: Minimize the number of Stakeholders' Requirements as much as possible}

Corollary 13: A Requirement of Designers is to have NIRs which are mutually independent.

Proof of Corollary 13: If NIRs were not independent between them because one DIR would affect more than one NIR, then Corollary 9 and Theorem 5 would not be satisfied. If NIRs were not independent between them because a CR would affect more than one NIR, then Theorem 6 would be contradicted.

The result of Corollary 13 is also a key point in Axiomatic Design (N. P. Suh 1990, 2001). However, for Axiomatic Design, the independence is explicitly established 1) by means of the very definition of the FRs (which must be independent in a neutral solution environment) and 2) by the formulation of the Independence Axiom (which states that FRs must keep their independence for the resultant design), whereas, for the framework of this thesis, independence is 
a necessary result that will apply to all the entities involved in the resultant design, not only to IRs.

This is a fundamental difference that will lead to a more general result (see Theorems 7 to 10).

Theorem 7: A Requirement of Designers is to minimize the number of dependences between all the Stakeholders' Requirements and hence, minimize the number of Stakeholders' Requirements.

Proof of Theorem 7: Corollary 13 prevents two different NIRs from sharing a common DIR and hence, it minimizes the number of dependences between NIRs. Theorem 5 also removes all dependences between DIRs contained in the DDM. Theorem 6 reduces the dependences to the ones between DIRs and their acceptance conditions and hence, removes all dependences between DIRs. Therefore, as per Property 13, the number of remaining dependences between Requirements is $r$ which, by Theorem 3, should be minimized. For that reason, the number of dependences has to be minimum. As for the number of Requirements, it's a consequence of minimizing the number of IRs (Theorem 3) and CRs (Theorem 6) since they are all the Rs.

Corollary 14: A Requirement of Designers is to minimize the number of dependences with customers' and suppliers' Requirements.

Proof of Corollary 14: Theorem 7 reduces the number of dependences between Stakeholders' Requirements and hence, as long as customers and suppliers are Stakeholders, the theorem holds for them.

\subsubsection{Theorem 8: Make the output as insensitive as possible}

Theorem 2 shows that a Requirement of Designers is to make all IRs' acceptance intervals as large as possible. Thus, following Corollary 6, they will try to convert them into CRs (or to remove them). However, as per Definition 10, there will always be at least one NIR and hence, an acceptance interval. For this reason, it is impossible to accomplish completely Corollary 1 and, in 
general, Designers have to face $|\mathbf{V}(\mathbf{x})|>0$. Attending to the result of Theorem 2 (see also Corollary

2) $|\mathbf{V}(\mathbf{x})|$ is reduced by increasing the length of the acceptance intervals but, as long as Designers accept that they will remain finite, $\mathbf{V}(\mathbf{x})=0$ cannot be strictly achieved. Next results come from the fact that Theorem 2 cannot be completely satisfied using Corollary 7.

Corollary 15: A Requirement of Designers is $\boldsymbol{a}=\mathbf{0}$ in Eq. 27.

Proof of Corollary 15: Assuming that Theorem 4 is satisfied, the worst deviation of the NIR number $i$ can be written from Eq. 27 as (note that due to the Postulate the variables $x_{i}$ are stochastic variables which have -1 and +1 as their extreme values and that $V_{i}(\mathbf{x})$ is unknown)

$$
\begin{gathered}
\left.y_{i}\right|_{\text {max }}=a_{i}+\sum_{j=1}^{q}\left|A_{i j}\right|+V_{i}\left(\mathbf{x}_{\text {max }}\right) \leq 1 \\
\left.y_{i}\right|_{\text {min }}=a_{i}-\sum_{j=1}^{q}\left|A_{i j}\right|+V_{i}\left(\mathbf{x}_{\text {min }}\right) \geq-1
\end{gathered}
$$

In the previous expressions (30 and 31 ), the variable $x_{j \max } \in\{-1,1\}$ is such that $\max \left(a_{i}+\sum_{j=1}^{q} A_{i j} x_{j}\right)=a_{i}+\sum_{j=1}^{q} A_{i j} x_{j \max }=a_{i}+\sum_{j=1}^{q}\left|A_{i j}\right|$ and $x_{j \min } \in\{-1,1\}$ is such that $\min \left(a_{i}-\sum_{j=1}^{q} A_{i j} x_{j}\right)=a_{i}-\sum_{j=1}^{q} A_{i j} x_{j \min }=a_{i}-\sum_{j=1}^{q}\left|A_{i j}\right|$. The restriction imposed by expressions 30 and 31 can be condensed into inequality 32.

$$
\sum_{j=1}^{q}\left|A_{i j}\right| \leq \min \left(1-a_{i}-V_{i}\left(\mathbf{x}_{\max }\right), 1+a_{i}+V_{i}\left(\mathbf{x}_{\min }\right)\right)
$$

Left-hand side of inequality 32 is always positive or equal to zero since it results from the addition of absolute values. Thus, right-hand side of the inequality has to define a non-negative interval.

$$
1-a_{i}-V_{i}\left(\mathbf{x}_{\max }\right) \geq 0 \Rightarrow a_{i} \leq 1-V_{i}\left(\mathbf{x}_{\max }\right)
$$




$$
1+a_{i}+V_{i}\left(\mathbf{x}_{\min }\right) \geq 0 \Rightarrow a_{i} \geq-1-V_{i}\left(\mathbf{x}_{\min }\right)
$$

Inequalities 33 and 34 can be condensed into the following relation:

$a_{i} \in\left[-1-V_{i}\left(\mathbf{x}_{\min }\right), 1-V_{i}\left(\mathbf{x}_{\max }\right)\right]$. For example, if Corollary 1 is satisfied, then $V_{i}\left(\mathbf{x}_{\max }\right) \rightarrow 0$,

and $V_{i}\left(\mathbf{x}_{\min }\right) \rightarrow 0$. If, in addition $a_{i}=1$, inequality 32 yields

$$
\sum_{j=1}^{q}\left|A_{i j}\right|=0 \Rightarrow A_{i 1}=\ldots=A_{i q}=0
$$

Eq. 35 contradicts Corollary 10 since it establishes a zero row in the design matrix (the full rank of the matrix would be lost). Note that $a_{i}= \pm 1$ should be averted because it can only be satisfied when $V_{i}\left(\mathbf{x}_{\max }\right)$ and $V_{i}\left(\mathbf{x}_{\min }\right)$ are zero, what cannot be strictly achieved. Inequalities 32 and 34 lead to $V_{i}\left(\mathbf{x}_{\max }\right)-V_{i}\left(\mathbf{x}_{\min }\right) \leq 2$, but as long as these variations are unpredictable we cannot assume any preferable sign or value for them. For that reason the point $a_{i}=0$, equidistant from the forbidden points $a_{i}=1$ and $a_{i}=-1$, is the option which can stand the largest unpredictable variations (Theorem 1).

The equality $a_{i}=0$ was also obtained in Benavides and Rodríguez (2013) and converts inequality 32 into

$$
\sum_{j=1}^{q}\left|A_{i j}\right| \leq \min \left(1-V_{i}\left(\mathbf{x}_{\max }\right), 1+V_{i}\left(\mathbf{x}_{\min }\right)\right)
$$

Note that Theorem 5 tends to reduce the number of non-zero elements present in a DDM row to one (the minimum number ensured by Definition 10), what tends to increase the range where inequality 36 holds. Assuming that Theorem 5 is fulfilled, inequality 36 yields

$$
\left|A_{i i}\right| \leq \min \left(1-V_{i}\left(\mathbf{x}_{\max }\right), 1+V_{i}\left(\mathbf{x}_{\min }\right)\right)
$$


Corollary 16: A Requirement of Designers is to have $\left|A_{i i}\right|$ as small as possible and, in any case, it cannot be higher than one.

Proof of Corollary 16: Note that, due to Theorem 1, a Requirement of Designers is to maximize the variation the output can stand but, according to inequality 35 , the value of $A_{i i}$ which stands the maximum dimensionless variations is zero. In addition, if $\left|A_{i i}\right|$ is bigger than one, then DIRs (which vary between -1 and 1) will be transformed into NIRs whose values will lie outside the interval $[-1,1]$ and hence, this will contradict the acceptance criteria of Property 11.

Theorem 8: A Requirement of Designers is to minimize the sensitivity. (Alternate statement: A Requirement of Designers is to make $\partial f_{i}\left(m_{0}\right) / \partial m_{j}$ as close to zero as possible).

Proof of Theorem 8: By Theorem 5, the terms which are not in the diagonal are zero $\left(A_{i j}=0\right.$ when $i \neq j$ ), proving the theorem for them because (due to fluctuation and feasibilityattributes) it can be only achieved if $\partial f_{i}\left(m_{0}\right) / \partial m_{j}=0$. For the terms in the diagonal, $A_{i i}=\left(\bar{m}_{i}-\underline{m}_{i}\right)\left(\partial f_{i}\left(m_{0} ; \mathbf{n}\right) / \partial m_{i}\right) /\left(\bar{l}_{i}-\underline{l}_{i}\right)$ (Eq. 29), Corollary 16 shows that $A_{i i}$ should tend to zero and Corollaries 2 and 3 show that $\bar{l}_{i}-\underline{l}_{i}$ and $\bar{m}_{i}-\underline{m}_{i}$ should tend to infinity. However, as long as the requirement given by Corollary 7 is removed, $\bar{l}_{i}-\underline{l}_{i}$ is increased until a finite value is eventually reached. Hence, the only way to satisfy $A_{i i} \rightarrow 0$ with $\bar{m}_{i}-\underline{m}_{i} \rightarrow \infty$ is to have a derivative tending to zero. In addition, when $\bar{m}_{i}-\underline{m}_{i}$ is increased until a finite value is eventually reached, $A_{i i} \rightarrow 0$ is satisfied thanks to $\bar{l}_{i}-\underline{l}_{i} \rightarrow \infty$, which is valid only if $\bar{l}_{i}-\underline{l}_{i}$ has not reached a finite value. This last step is forbidden by hypothesis (the requirement of Corollary 7 is removed) and, again, $A_{i i} \rightarrow 0$ forces the derivative to tend to zero. 
The result stated by Theorem 8 was also reported by El-Haik (2005). Indeed, it is also a result that can be derived from the minimization of the Quality Loss Function (Taguchi, Elsayed and Hsiang 1989).

\subsubsection{Theorem 9: Minimize the number and the strength of the dependences on unpredictable variations as much as possible}

Theorem 9. A Requirement of Designers is to minimize the number and the strength of the dependences on unpredictable variations.

Proof of Theorem 9: In this work unpredictable variations are present a) in the DM through the terms $\boldsymbol{\delta}_{\mathbf{f}}(\mathbf{m} ; \mathbf{n}), \boldsymbol{\delta}_{\mathbf{g}}(\mathbf{m} ; \mathbf{n})$ and $\boldsymbol{\delta}_{\mathbf{h}}(\mathbf{m} ; \mathbf{n})$ in Eq. 20 to 23 and b) in the statistical moments associated to the DIs through $\boldsymbol{\delta}_{\mathbf{m}}$ in Eq. 24. Regarding a) Corollaries 1 and 2 and Theorems 3 and 6 minimize the number and strength (also by Corollary 15) of the dependences with respect to $\boldsymbol{\delta}_{\mathbf{f}}(\mathbf{m} ; \mathbf{n}), \boldsymbol{\delta}_{\mathbf{g}}(\mathbf{m} ; \mathbf{n})$ and $\boldsymbol{\delta}_{\mathbf{h}}(\mathbf{m} ; \mathbf{n})$. Asfor b) Corollaries 1,3 and 4 and Theorem 3 reduce the number and strength (also by means of Corollary 15) of these dependences. In addition, the DM is a constant (Theorem 4), full-rank diagonal matrix (Theorem 5) and as near zero as possible (Corollary 16 and Theorem 8). Consequently, only the first statistical moments of a reduced (Theorem 3) number of DIs, whose acceptance intervals are as wide as possible, are contemplated in the DM. This ensures that the strength and number of the dependences on the unknown statistical moments associated to the DIs are minimum.

\subsubsection{Theorem 10: Minimize the number and the strength of all dependences as much as possible}

Theorem 10: A Requirement of Designers is to minimize the number and strength of all 
dependences.

Proof of Theorem 10: The proof has two parts: 1) to prove that a Requirement of Designers is to minimize the number of all dependences as much as possible, and 2) to prove that a Requirement of Designers is to minimize the strength of all dependences as much as possible.

Part 1: Definition 8 states that all dependences are contained in the RM which can be divided into a) the DDM and a (see Eq. 27) as per Theorem 4, b) the CE and DM 2 and c) the unknown dependences (caused by unpredictable variations) represented by $\boldsymbol{\delta}_{\mathbf{f}}(\mathbf{m} ; \mathbf{n}), \boldsymbol{\delta}_{\mathbf{g}}(\mathbf{m} ; \mathbf{n})$ and $\boldsymbol{\delta}_{\mathbf{h}}(\mathbf{m} ; \mathbf{n})$. Firstly, for a) Theorems 5 and 7 reduce as much as possible the number of components in the matrix and hence, the number of dependences and Corollary 15 reduces the number of components in a down to zero. As for b) Theorem 6 proves that a Requirement is to minimize the number of the dependences contained in the CE and DM. Finally, regarding c) Theorem 9 proves that Designers want to remove the number of dependences on unpredictable variations and hence, on $\boldsymbol{\delta}_{\mathbf{f}}(\mathbf{m} ; \mathbf{n}), \boldsymbol{\delta}_{\mathbf{g}}(\mathbf{m} ; \mathbf{n})$ and $\boldsymbol{\delta}_{\mathbf{h}}(\mathbf{m} ; \mathbf{n})$. Consequently, we can conclude that a Requirement of Designers is to reduce the number of (all) dependences as much as possible.

Part 2: As for a), Theorem 8 minimizes the strength of dependences contained in the DDM and Corollary 15 makes a (see Eq. 27) equal to zero, regarding b) Theorem 6 minimizes the strength of the dependences contained in the CE and $\mathrm{DM}_{2}$ and as to c) Theorem 9 minimizes the strength of the dependences on unpredictable variations.

Next Figure 2 shows the relations between Theorems, Corollaries, Definitions and Properties. Each box has influences from the previous boxes and labels to which it is connected. 


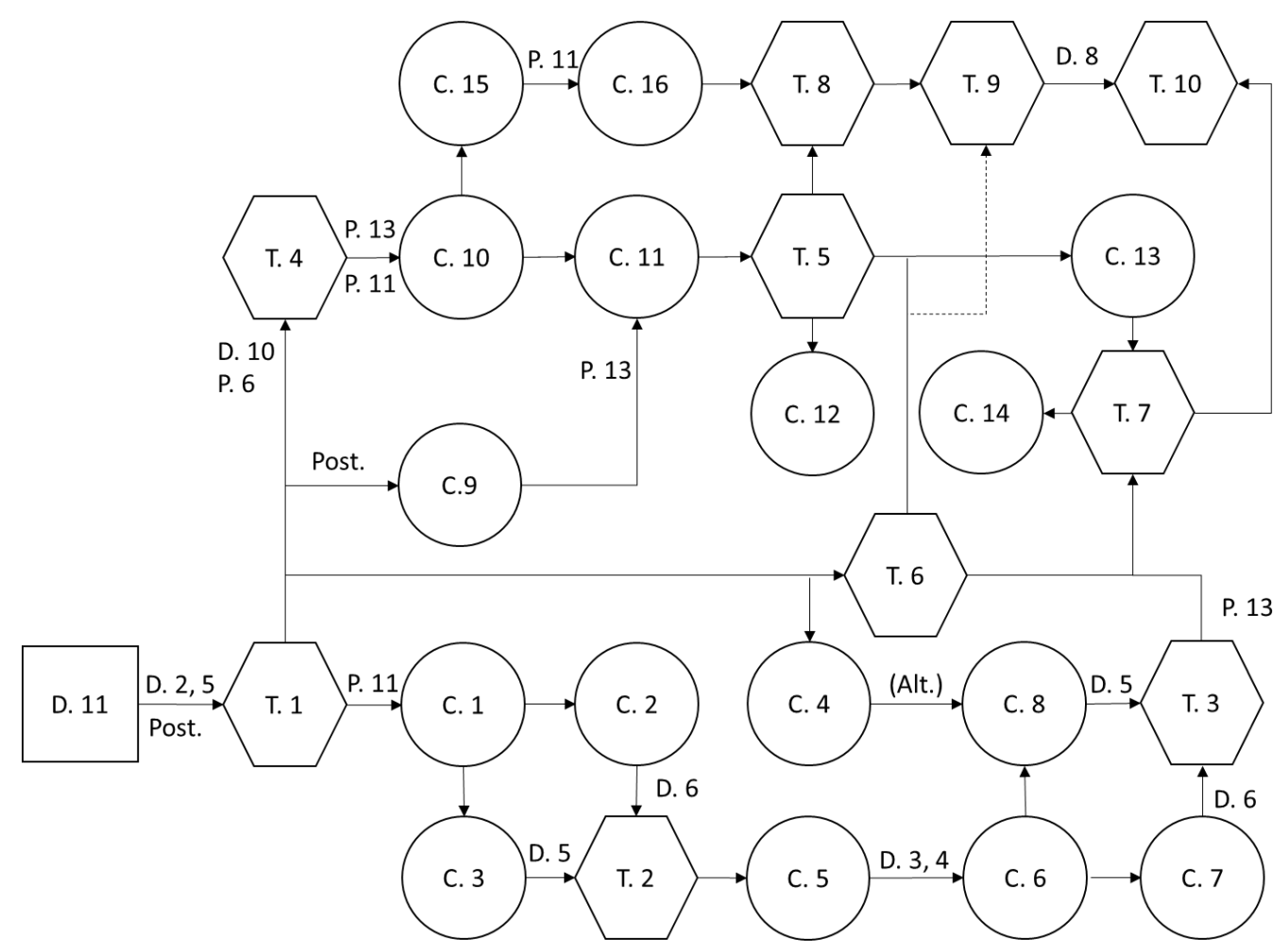

Figure 2: Relations between theorems, corollaries, properties and definitions

\subsubsection{Theorem 11: Minimizing the number and strength of dependences makes the output robust}

Theorem 11: Assuming that the Postulate and Definitions 1 to 11 hold, Theorem 1 holds if and only if Theorem 10 holds.

Proof of Theorem 11: It has already been proven that Theorem 1 leads to Theorem 10 so, for completely demonstrating Theorem 11, it is necessary to prove also that Theorem 10 entails Theorem 1. Theorem 10 implies that the Requirement is that all dependences have to be minimized in number and in strength. As long as the dependences are all ways of affecting the output, the dependences between the output and unpredictable variations are minimized in number and strength. This minimizes the impact of unpredictable variations on the acceptance (according to Definition 2) of the output. Since Theorem 10 maximizes the probabilities of 
acceptance in the presence of unpredictable variations, it also maximizes the output's characteristic of robustness. In addition, Theorem 10 avoids having another output with fewer dependences and less strength and hence it implies that the output features a global maximum for robustness. Hence, Theorem 10 implies Theorem 1.

This theorem is a key point of the framework presented in this thesis. This result arises from the new starting point adopted. In particular, it is derived from the definitions of the Stakeholders' Rs, IRs, CRs, the IRDDM and the Postulate. A more convenient formulation of Theorem 11 is given as the following Corollary.

Corollary 17: The following two Rs are equivalent: "minimize the number and strength of all dependences" and "make the output robust".

Proof of Corollary 17: It is the consequence of introducing the wording of Theorem 1 and Theorem 10 into Theorem 11.

This result states that minimizing the number and strength of dependences is the necessary and sufficient condition for robustness, or in other words, the Requirement of making robust outputs is equivalent to the Requirement of making outputs with the lowest number and weakest strength of dependences.

\subsubsection{Theorem 12: Minimizing the number and strength of dependences increases the output value}

In the literature different definitions of lifetime can be found. For example, Engel et al. (2016) define it as the time at which the degradation first reaches the failure threshold and Mueller and Ganseforth (2012) discuss the difference between the market-focused (Nedeau and Casselman 2008) and the extended lifetime (Faneye 2005). Other authors, like Umeda and Life Cycle Design Committee (2001) and Kobayashi (2000, 2005) use the absolute lifetime, which is based on 
product failure and physical degradation, and the value lifetime, which is defined by when the product is disposed because its performance, function or appearance does not satisfy the changed customer preferences. Based on this last definition, the following definition is provided:

Definition 12: The lifetime is the period of time during which the output satisfies the acceptance criterion in the sense of Definition 2.

Corollary 18: Minimizing the number and strength of dependences increases the lifetime of the output.

Proof of Corollary 18: Theorem 11 ensures that minimizing the number and strength of dependences maximizes the robustness of the output and hence, by Definition 11, the acceptance criterion is ensured with the maximum achievable probability. The more the output is out of their acceptance limits, the shorter its lifetime because the less time it fulfils the acceptance criterion.

Corollary 19: Assuming that the cost of minimizing the number and strength of dependences is negligible, reducing (increasing) the number and strength of dependences reduces (increases) the output cost.

Proof of Corollary 19: Reducing (increasing) the number of dependences reduces (increases) the required cost for understanding and controlling those dependences in such a way that the satisfaction of the acceptance conditions can be ensured. Reducing (increasing) the strength of the dependences reduces (increases) the cost for understanding and controlling those dependences because they affect in a more (less) relevant way the acceptance conditions. Therefore, an output which satisfies Theorem 10 reduces its cost.

Corollary 19 can be explained attending to the following argumentation: minimizing the number of dependences reduces the number of relations to be understood, implemented, and 
ensured during the whole lifetime of the output, and reducing its strength reduces the effort devoted to controlling them, which reduces the amount of information managed and its associated costs, leading this to a cost reduction during the design and manufacturing processes. In addition, the assumption of having a negligible cost for the minimization holds when a) the designer imposes Theorem 10 as a HR since the very beginning of the design process and b) the creativity process is good enough.

As Mueller and Ganseforth (2012) explain, the main target of the resultant design is the technically and economically optimal solution to a given set of Rs. Based on this, the concept of output value is introduced:

Definition 13: Output value is a characteristic which permits to classify outputs as better or worse in the following way: if two outputs a) have the same set of NIRs and NCRs and b) fulfil the acceptance criterion in the sense of Definition 2, then one output is more valuable than the other precisely when the former has a higher lifetime and a lower cost than the later (ceteris paribus with respect to other elements which may affect output value).

Output value can be affected by different aspects, such as lifetime, cost, price, subjective perceptions..., which subsequently affect satisfaction. However, the framework of this study only addresses two parameters: lifetime and cost. For this reason, the rest of the parameters are fixed. This leads to the inclusion of the premises a) and b) in Definition 13 in order to avoid contradictions with other definitions of value such as the one given by Juran and Gryna (1988).

Theorem 12: Assuming that the cost of minimizing the number and strength of dependences is negligible, Theorem 10 increases the output value (according to Definition 13).

Proof of Theorem 12: The output which satisfies Theorem 10 has a higher output value since Corollary 18 says that Theorem 10 increases the lifetime of the output and Corollary 19 proves 
that Theorem 10 reduces its cost. Hence, as per Definition 13, Theorem 10 increases the resultant value of the design.

For example, if there are two different outputs for a refrigeration machine which only differ in the coolant, such that Design A employs water and Design B arsenic, then Design B sets a new dependence with respect to the environment (arsenic is a potential pollutant) which Design A does not have. Consequently, Design A is a more valuable output.

Theorem 12 is a value proposition which states that Theorem 10 is also a value proposition (with the definition of output value provided in Definition 13) and, attending to Theorem 11, we can conclude that Theorems 1 to 9 are also value propositions. For example, increasing the acceptance intervals (Theorem 2), minimizing the number of IRs (Theorem 3), and reducing the sensitivity between variables (Theorem 8 ) lead to minimize the dependences and hence to increase the output value. In the framework of the definitions and the postulate adopted, the connection with output value is objective since it comes directly from Corollaries 18 and 19: if Theorem 10 is applied, then the lifetime is increased and the cost reduced.

\subsubsection{Theorem 13: The Ideal Output}

It is interesting to note that Theorems 1 to 10 have been written as DCRs and thus, the following conflict arises: Theorem 6 claims to reduce the CRs, but Theorems 1 to 10 are ten new DCRs which were not previously considered and increase the number of CRs. The same conflict appears between Corollary 9 and Theorem 5 because the later prevents the removal of any additional dependence in order to avert the singularity of the DDM. However, such conflicts are not contradictions; they only anticipate the existence of a minimum number of DCRs and, in general, of dependences. Note that Theorems 11 and 12 allow us to reduce 10 DCRs to 1 , which is the one derived from Theorem 10. It is interesting to note that this Theorem 10 can be considered 
as an HR or SR depending on the Designers viewpoint. For some Designers (attending to Theorem 12) the fulfilment of Theorem 10 could be so important that if it is not satisfied, the resultant design is rejected (HR) and for others it may be a nice-to-have characteristic in such a way that its fulfilment is not necessary for accepting the resultant design (SR). Again, this assessment will depend on the particular design scenario.

From the discussion up to this point, it can be observed that the output is not an empty set and for the same reason, the minimum number of dependences is not zero. The following definitions and theorems describe this minimum. This output is named as the Ideal Output since it is the utopic situation described by the Designers' Requirements.

Definition 14: The Ideal Output satisfies the acceptance criterion of Definition 2 and it is described by the DCR imposed by Theorem 10.

Corollary 20: The Ideal Output satisfies the acceptance criterion and it is described by the set of DCRs contained in Theorems 1 to 10.

Proof of Corollary 20: Based on Definition 14, the DCR which describes the Ideal Output is Theorem 10. However, Theorem 11 states that Theorem 1 comes from Theorem 10, being Theorem 1 the one from which Theorems 2 to 10 are generated.

Corollary 21: Given a fixed set of NIRs and sensitivities, the Ideal Output satisfies the acceptance criterion and fulfils 1) the minimum number of DIRs is $r$, 2) the dependences between NIRs and DIRs are one to one, 3) the dependence between one NIR and its associated DIR is linear such that a (Eq. 27) is zero, 4) the minimum number of CRs is 4r (2r acceptance CRs for each set of DIRs and NIRs), 5) the minimum number of dependences is $5 r$, (r connecting DIRs to NIRs, and $4 r$ representing the two acceptance CRs for each of the $2 r$ previous IRs) and 6) NIRs' and DIRs' acceptance intervals are as wide as possible. 
Proof of Corollary 21: Points 1) and 2) are a direct result of Theorems 4 and 5. Point 3) stems from Theorems 4 and, 5 and Corollary 15. Point 4) is a direct result of Theorem 6 which tends to minimize CRs taking into account the acceptance conditions of the IRs. Point 5) is the result of the dependences set by the previous points and point 6) is a consequence of Theorem 2. At that point, Theorem 10 is not necessary as long as there is not any other dependence which can be removed or weakened.

This result, despite similar, is not equal to the one reported by N. P. Suh $(1990,2001)$ since now the linearity and the reduction of CRs are necessary ingredients of the Ideal Output.

Corollary 22: The Ideal Output satisfies the acceptance criterion and fulfils 1) the number of DIRs is one, 2) the dependence between the NIR and the DIR is linear such that a (Eq. 27) is zero, 3) the sensitivity of NIR to DIR is as low as possible, 4) the minimum number of CRs is four, 5) the number of dependences is five and 6) NIRs' and DIRs' acceptance intervals are as wide as possible.

Proof of Corollary 22: It is the result of applying Theorem 10 to the premise of Corollary 21 (i.e. "given a fixed set of NIRs and sensitivities" is removed): if the number of NIRs is not fixed, then Theorem 10 will tend to reduce it to just one.

For any output different from the Ideal Output, Theorem 10 should be a Designers' Requirement. This ensures keeping the Ideal Output stable against decisions made during the design process. Note that Theorem 10 tends to reduce sensitivities and generates all the other theorems. Consequently, its permanent presence when dealing with non-Ideal Outputs prevents Stakeholders from changing the Requirements described by points 1) to 6) of Corollaries 20 and 21 during the design process. 
Theorem 13: For a given set of NIRs jand NCRs, the Ideal Output maximizes the output value (as per Definition 13).

Proof of Theorem 13: Attending to Definition 14, the Ideal Output satisfies the acceptance criterion. Besides, the number and strength of dependences (see Corollaries 20 or 21) cannot be reduced anymore and by Theorems 10 and 12, the output value is maximized.

Corollary 23: For a given set of NIRs and NCRs, the Designers' activity which provides the output with the most value (as per Definition 13) is to seek the Ideal Output.

Proof of Corollary 23: Attending to Theorem 13, the Ideal Output maximizes the output value thus, seeking the Ideal Output is the activity which reports the most value to the output.

Corollary 24: The minimum number of dependences which generate the value of an output (as per Definition 13) is 5r, where $r$ is the number of NIRs.

Proof of Corollary 24: Theorem 13 states that the Ideal Output maximizes the output value and for a given set of NIRs, according to Corollary 21, has the minimum number of dependences, which is 5 r (according again to Corollary 21).

Corollary 24 shows that there are dependences which support output value and thus, are not removable. This allows us to give the following definitions:

Definition 15: Needs are the dependences which generate the value of an output (as per Definition 13).

Definition 16: Constraints are the dependences which do not generate the value of an output (as per Definition 13).

Corollary 25: Constraints reduce the output value (as per Definition 13).

Proof of Corollary 25: Constraints are dependences which do not generate value in the output and hence, they can be removed without losing this value. As per Theorem 12 and through 
Theorem 10, if they are removed, they increase the value of the output. Therefore, Constraints reduce the value with respect to the output with the same Needs but without any Constraints.

Corollary 26: During the design process, the removal of Constraints increases more the output value (as per Definition 13) than trying to redesign in order to accommodate them.

Proof of Corollary 26: Corollary 25 states that constraints reduce output value and they should be removed. If not, accommodating these constraints would require to increase the number of dependences what would also reduce the output value (Theorem 12).

The results obtained in this section describe the utopic target that designers should try to find. As long as $\{\mathrm{Rs}\}$ (including both DRs and NRs) evolve in a design process, the degree of fulfilment of these Theorems and Corollaries also evolves. If the new set of Rs leads to a better degree of fulfilment, the new stage of design is more suitable than the previous one and should be adopted. The best set of Rs is the one that allows the designer to implement the Ideal Output (as per Corollary 22).

\subsection{Discussion about Corollary 3 and Theorem 8}

As explained in Corollary 16, Designers should take into account that reducing the absolute value of $A_{i i}$ as much as possible is very important for maximizing the robustness of the resultant design and that the absolute value of $A_{i i}$ cannot be bigger than 1 . However, when $\bar{m}_{i}-\underline{m}_{i}$ and $\bar{l}_{i}-\underline{l}_{i}$ have upper bounds and $\partial f_{i}\left(m_{0}\right) / \partial m_{i}$ has a lower bound, new dependences can appear as the next Corollary shows.

Corollary 27: If $\bar{m}_{i}-\underline{m}_{i}$ and $\bar{l}_{i}-\underline{l}_{i}$ have upper bounds and $\partial f_{i}\left(m_{0}\right) / \partial m_{i}$ has a lower bound, then 1) the value reached by the output is lower than the one of the Ideal Output,2) the use of Theorem 8, which reduces the lower bound of $\partial f_{i}\left(m_{0}\right) / \partial m_{i}$, generates more value than the use 
of Corollary 3, which increases the upper bound of $\bar{m}_{i}-\underline{m}_{i}$, and 3) the more the upper bound of $\bar{m}_{i}-\underline{m}_{i}$ increases and the more the lower bound of $\partial f_{i}\left(m_{0}\right) / \partial m_{i}$ decreases, the more the value approaches the one of the Ideal Output.

Proof of Corollary 27: According to Corollary 16, a Requirement of Designers is $A_{i i} \rightarrow 0$. However, when $\bar{m}_{i}-\underline{m}_{i}$ and $\bar{l}_{i}-\underline{l}_{i}$ have upper bounds and $\partial f_{i}\left(m_{0}\right) / \partial m_{i}$ has a lower bound, $A_{i i} \rightarrow 0$ cannot be achieved, what leads to a dependence between $\bar{m}_{i}-\underline{m}_{i}, \bar{l}_{i}-\underline{l}_{i}$ and $\partial f_{i}\left(m_{0}\right) / \partial m_{i}$. This dependence is illustrated in Table 2 which, according to Theorem 10, should be as weak as possible or completely removed. As long as this cannot be achieved, Theorem 12 states that the output value is lower than the Ideal Output's. This proves part 1. As shown in Table 2, the dependence created when only Theorem 8 is used is weaker than the one generated when only Corollary 3 is used. Thus, Theorem 12 proves part 2. In addition, as long as increasing the upper bound of $\bar{m}_{i}-\underline{m}_{i}$ and reducing the lower bound of $\partial f_{i}\left(m_{0}\right) / \partial m_{i}$ permit to satisfy in a larger extent Corollary 3 and Theorem 8, the output approaches more the Ideal Output, which, attending to Theorem 13, is the one with the highest output value. This proves part 3. 


\begin{tabular}{|c|c|c|c|c|c|c|}
\hline \multirow[b]{2}{*}{$\begin{array}{l}\text { Design } \\
\text { rules }\end{array}$} & \multicolumn{4}{|c|}{ Cases } & \multirow{2}{*}{$\begin{array}{c}\text { Likelihood of } \\
\text { satisfying } \\
\Delta m_{i} \frac{\partial f_{i}}{\partial m_{i}} \ll \Delta l_{i}\end{array}$} & \multirow{2}{*}{$\begin{array}{c}\text { Dependence } \\
\text { between } \Delta l_{i} \\
\text { and } \Delta m_{i}\end{array}$} \\
\hline & $\Delta m_{i}$ & $\frac{\partial f_{i}}{\partial m_{i}}$ & $\Delta m_{i} \frac{\partial f_{i}}{\partial m_{i}}$ & $\Delta l_{i}$ & & \\
\hline None & Ref. & Ref. & Ref. & Ref. & Ref. & Yes (Ref.) \\
\hline Cor. 3 & Bigger & Ref. & Bigger & Ref. & Lower & Yes (Stronger) \\
\hline Th. 8 & Ref. & Smaller & Smaller & Ref. & Higher & Yes (Weaker) \\
\hline Ideal & Bigger & $\rightarrow 0$ & $\rightarrow 0$ & Bigger & 1.0 & No \\
\hline
\end{tabular}

Note: $\Delta m_{i}=\bar{m}_{i}-\underline{m}_{i}$ and $\Delta l_{i}=\bar{l}_{i}-\underline{l}_{i}$.

Ref. are the reference values of each column. 'None' fixes the reference values and it indicates that none of the rules obtained in this thesis to characterize the Ideal Output has been used.

Table 2: Effects of Corollary 3 and Theorem 8 on the dependence between the limits of acceptance under different sets of design rules

\subsection{Comments on Axiomatic Design}

Axiomatic Design (N. P. Suh 1990, 2001) is a design methodology which presents designing as a relation between what the designer wants to obtain (Functional Requirements or FRs) and how to obtain it (Design Parameters or DPs) in the presence of internal and system constraints (Cs). As a part of the methodology, Axiomatic Design follows a zig-zagging process that converts the DPs of the previous step into the FRs of the next one. Two axioms are the cornerstone of Axiomatic Design:

- Independence Axiom: Maintain the independence of FRs.

- Information Axiom: Minimize the information content of the design. 
FRs from Axiomatic Design (N. P. Suh, 1990) are defined as the minimum set of independent requirements (this independence is stated in a neutral solution environment, i.e. without considering the DPs) that completely characterizes the design objectives for a specific need. Cs are defined as bounds on an acceptable solution and, by definition, a $\mathrm{C}$ in Axiomatic Design does not have to be independent of other Cs and FRs. This means that the set of Cs is another proper subset of Rs whose intersection with the subset of FRs is empty.

Axiomatic Design defines information content as $I=-\log (\|f(D D) \cap N D\| /\|f(D D)\|)$, where the denominator is the hyper-volume (length for a one dimensional problem) of the system range $f(D D)$ and the numerator is the hyper-volume of the common range, i.e. the intersection of $f(D D)$ and the design range ND. Thus, the information content $I=-\log p$ is the uncertainty associated to a probability of success equal to $p$ (N. P. Suh 1990, 2001). N. P. Suh assumes (by gathering sufficient evidences) that these axioms lead to the best outputs. In the framework elaborated in this thesis these axioms can be deduced as the following theorems:

Theorem 14: Independence Axiom - Maintain the independence of FRs.

Proof of Theorem 14: Attending to Theorem 7, the number of dependences between FRs must be minimum. Since FRs are a subset of Rs, this theorem ensures that FRs must be (independent and) kept independent.

Theorem 15: Information Axiom - Minimize the information content of the output.

Proof of Theorem 15: The minimum of the information content is obtained when $f(D D) \subseteq N D$ because in that situation $f(D D) \cap N D=f(D D)$ and hence, the information content is zero. However, $f(D D) \subseteq N D$ is established by Properties 10, 11 and 12 as a part of the acceptance criteria. Therefore, these properties imply a zero value for information content, what proves the theorem. 
Theorem 16: The following relations hold: 1) $\{F R\} \varsubsetneqq\{R\}, 2)\{D P\} \varsubsetneqq\{R\}, 3)\{C\} \varsubsetneqq\{R\}$ and 4) $\{C\} \cap\{F R\}=\varnothing$.

Proof of Theorem 16: Rs contain FRs but also Cs and DPs, which proves 1, 2 and 3. By definition, Cs are not FRs, what proves 4.

It is interesting to note that N. P. Suh (1990) states that a fundamental difference between Cs and FRs is that normally FRs have tolerances whereas Cs do not, what evidences that FRs tend to be IRs, which, attending to Theorem 3, have to be a minimum set (as required by the N. P. Suh's definition of FRs). In the context of this work, if the Postulate and definitions in Sections 2.2 to 2.6 are accepted, Independence and Information Axioms have been proved to be Theorems 14 and 15. This means that robustness implies Independence and Information Axioms. This is a powerful result because it proves that Axiomatic Theory is a necessary ingredient for robustness (in the sense of Definition 11) and for value (in the sense of Definition 13), what makes it a very valuable theory for designing. However, in the framework developed here, the converse does not hold and hence Axiomatic Design does not always have to lead to the maximum robustness and value if other aspects are not taken into consideration.

Theorem 17: Axiomatic Design is a necessary, but not sufficient, condition for robustness (according to Definition 11) and value (according to Definition 13).

Proof of Theorem 17: As stated by Theorem 11, achieving robustness (i.e. the converse) requires the minimization of all the dependences, what also includes the dependences involving Cs and not only the ones between the FRs and DPs.

Theorem 18: In the framework presented in this thesis, the Independence Axiom is a logical consequence of the Information Axiom. 
Proof of Theorem 18: As Theorem 14 shows, the Independence Axiom is a logical result which comes from Corollary 13. Proving Corollary 13 requires Theorem 5 which requires as well Property 11 through Corollary 10 (see Fig. 2). Property 11 is directly a statement of the Information Axiom as per Theorem 15. This proves that the Information Axiom precedes in the logical argumentation (and it is necessary) to prove the Independence Axiom.

Theorem 18 does not contradict the result of El-Haik and Yang (1999), which states that both axioms are necessary since, as explained by N. P. Suh (2001), each one addresses a different conceptual design weakness. In this thesis, the notion of acceptance (Definitions 1 to 10) and the notion of unpredictable variations (Postulate) are stated as necessary concepts and, as a consequence, the two axioms appear as a logical result. Thus, two different concepts are still used to build the two axioms.

The acceptance criterion given to the IRs (Definition 3) would be a $\mathrm{C}$ for Axiomatic Design, and hence the Independence Axiom does not apply to them: they are only the object of the Information Axiom, which does not lead in a general case to conclude that the dependences on the acceptance intervals must also be minimized. Following the description made in this thesis, the two axioms can be considered as DCRs embedded in a DM, say $\mathrm{DM}_{\mathrm{A}}$. Now $\mathrm{DM}_{\mathrm{A}}$ and Theorem 10 can be embedded in another DM, say $\mathrm{DM}_{\mathrm{B}}$. Both DMs will eventually lead to acceptable resultant designs by Stakeholders but, since Axiomatic Design is less restrictive than Theorem 10, the result does not have to be the same. As Theorem 17 proves, any result of the new theory should also be a solution of Axiomatic Design but not all the solutions from Axiomatic Design will be solutions of this new study. Other minor differences between both approaches are:

- The DDM, defined in Eq. 28 and 29, differs from the Design Matrix defined by N. P. Suh (1990, 2001) because the DDM includes the lengths of Stakeholders' acceptance intervals 
whereas the Axiomatic Design's matrix is just the Jacobian, $\mathbf{J}\left(\mathbf{m}_{0}\right)$, of the transfer function. Thus, the Jacobian includes less information about the design than the DDM. In addition, every DDM component (given by Eq. 29) is a dimensionless number, allowing in this way Designers to compare each DDM component with the others and letting them decide if they can drop it as a negligible effect.

- The Ideal Output deduced in this work (Definition 14) gives more information (Corollaries 20 to 26) than Axiomatic Design does because, for example, it also concludes that the Ideal Output has to be linear (Theorem 4). A Linearity Theorem has been also reported by Benavides (2012) and Benavides and Rodriguez (2016), but their proof uses an external ingredient to the Axiomatic Design as well: the transformation of Cs (the bounds of the previous FRs) into new FRs.

- The framework of this study gives a main role to both the IRs and the CRs whereas in the Axiomatic Design theory only FRs have a main role.

- Axiomatic Design incorporates the design range through the Information Axiom whereas this study incorporates it in the DM.

\subsection{Conclusion}

This section has presented a theoretical study whose objective is to obtain a set of necessary and sufficient conditions for robustness in design scenarios where interval requirements are present. The premises on which the study relies are explicitly written in a postulate, stating the presence of unpredictable variability and uncertainty, and in the Interval Requirement-Driven Designers' Model definition. This type of analysis has the advantage of using a single postulate about variations (more general than other sets of qualitative design rules) and the advantage of 
using a qualitative approach (more suitable for conceptual design than a quantitative approach). The methodology adopted has focused on building a theoretical framework, based on logical consequences of the postulate and the definitions, as self-consistent and general as the postulate and the definitions permit. For this reason, it has been a fundamental part of the methodology the selection of the definitions used for the concepts involved in the argumentation and its connection with previous scientific literature. This approach to the problem allows readers to check whether these premises fit their particular situation or not and hence, whether the derived statements apply or not to their particular design cases.

The main theoretical results are Theorems 10 and 11: minimizing the number and the strength of all dependences as much as possible is a necessary and sufficient condition for robustness. This result leads to the definition of the Ideal Output, the one with the weakest and the lowest number of dependences. Thus, it is a fundamental result of the thesis that, when seeking robustness, all the dependences are important and all of them must be the target of the designers' labour. This is the main difference with respect to other previous design theories, which are only focused on particular sets of dependences. In addition, thanks to the inclusion of all the dependences, a notion of output value arises. This notion allows the study to conclude that there are two kind of dependences, those which generate the output value, called needs, and those which restrict or reduce it, called constraints. Therefore, Theorem 10 turns the notion of value more objective instead of more subjective and it links dependences directly to output robustness and output value.

The study has shown that there is at least one framework which arrives at a set of sufficient and necessary conditions for robustness. However, it does not conclude if this framework is the only possible one, what should be a topic for future works. The set of premises and definitions 
presented in this study has also allowed a generalization of certain concepts. However, some definitions are still quite restrictive and further research should be done in order to explore other sets of definitions which could lead to more general results. 


\section{A DESIGN METHODOLOGY FOR MINIMIZING DEPENDENCES}

\subsection{Introduction}

As explained in the introduction of this thesis, there seems to be a consensus in the literature about how important preliminary decision-making tools are. Therefore, the aim of this section is to present a design methodology which can help Designers attain the objectives of Section 2, which presented a set of necessary and sufficient conditions for robustness in design scenarios where interval requirements are present. After a set of theorems, the Ideal Output arises, condensed as 'it is the output which has the lowest and weakest number of dependences'. Therefore, the results of that section identified the output of the design process but not the design process in itself. However, as long as not all design processes can obtain the same objective, the aforementioned target will require a special set of activities.

The objective of this section is to present a methodology which can be used by designers in scenarios where they have to minimize dependences during the design process such as the one presented in Section 2 (and in this case, to obtain an output as close as possible to the one of the Ideal Design). To this end, a classification of dependences and costs are presented. The cost classification is especially relevant since dependences will be measured and evaluated through these costs. Then, a methodology is proposed which consists of a set of steps that minimize the different dependences in a sequential way, leading this to an output whose characteristics are as close as possible to the ones of the Ideal Design in case it is applied in a design scenario such as the one presented in Section 2. Other design scenarios may also benefit from this methodology but their identification is out of the scope of this study. 


\subsection{Black Box Items}

A relevant element in design methodologies is the basic brick used by Designers for constructing the whole output. Attending to the general properties described by Bunge (1963), the Black-Box Item gathers the characteristics required during the conceptual design. Theses BlackBox Items are aligned with the statement of Peder (1997): at concept stage, we are only concerned with the structure and arrangement of elements and the mode of action.

Definition 17: A Black-Box Item (BBI) is an item into which, according to Bunge (1963), a given stimuli impinge and out of which reactions emerge. The constitution and structure of the box are altogether irrelevant to the approach under consideration, which is purely external or phenomenological. Or, as this author states, only the behaviour of the system will be accounted for.

Hence, BBIs stablish dependences between the stimuli and reactions: when stimuli vary in a certain way, reactions will change accordingly. It is important to note that stimuli can come from different sources (e.g. users, environment...) and some will be predictable whereas others can be unpredictable, representing these last, for example, external perturbations which can affect the BBI response. Next Figure 3 shows the set of stimuli impinging on a BBI $\left(x_{1}, x_{2}, \ldots x_{a}\right)$ and the set of reactions emerging from it $\left(y_{1}, y_{2}, \ldots y_{b}\right)$.

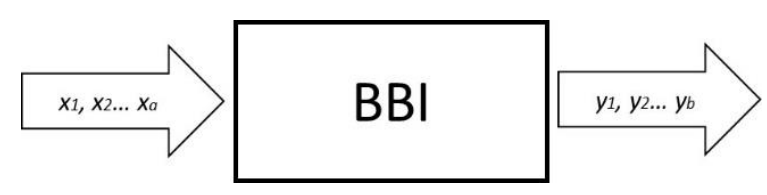

Figure 3: Black Box Item stimuli and reactions

BBIs represent the elements or systems (set of elements as per ISO 9000:2015) whose inside is not relevant to Designers. Consequently, the consideration of an item as a BBI is 
contingent upon the concrete design process. For example, a BBI can be a material, a resistor, a $\mathrm{PCB}$, a screen or a laptop depending on the level of detail required by Designers. Indeed, when designing a screen, a resistor can be considered as a BBI. However, when designing a control room, the screen can be now a BBI.

According to Definition 17, items taken as given by Designers are BBIs (e.g. the car's battery when designing a vehicle or a dynamic-link library when programming a code for Windows). In the same way, a workgroup in an organization can be a BBI for other groups of the organization (e.g. the purchasing department can be a BBI for the rest of the departments in the context of the organizational structure). This means they are not interested in the way how these elements work internally but only in what happens at their interfaces.

Figure 4 shows an example of an output composed of several BBIs. The nomenclature followed is such that $x_{(a), b}$ represents the $b$-th stimulus of the BBI $a$ and $y_{(a), b}$ the $b$-th reaction of the same BBI.

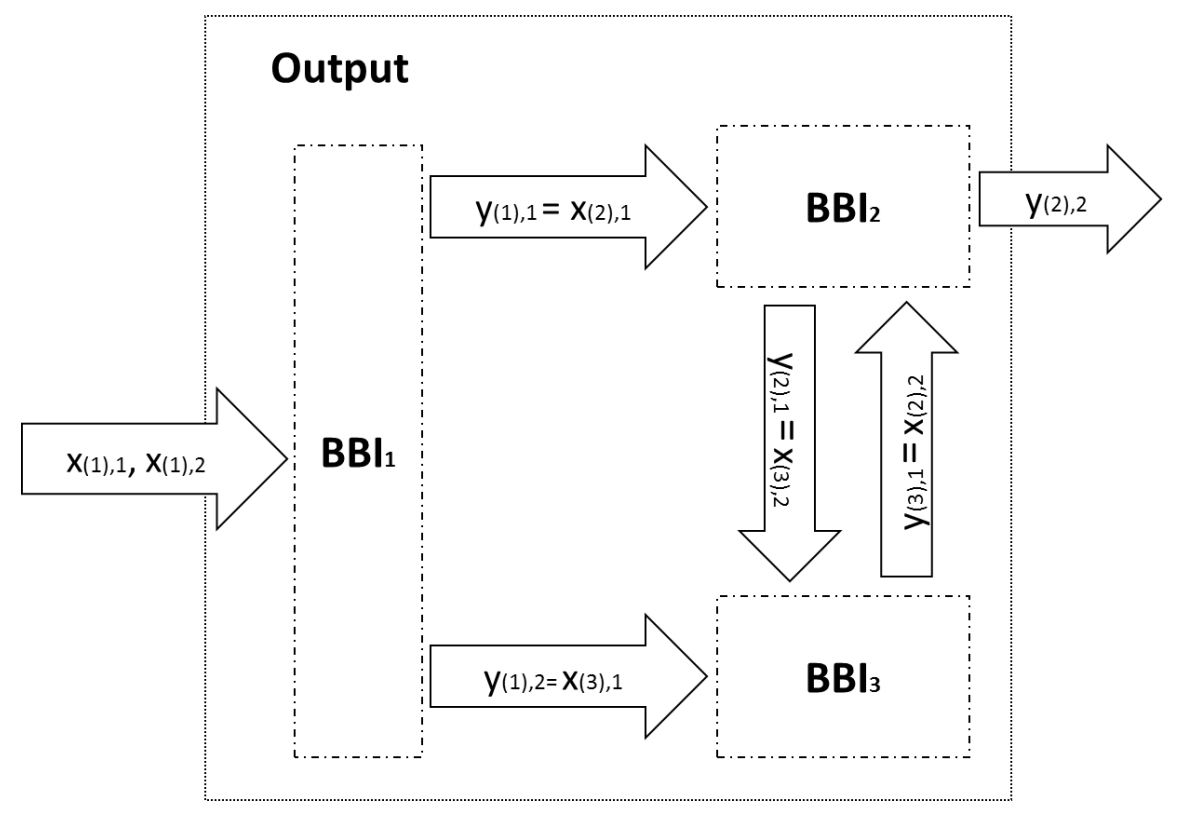

Figure 4: BBI composition example 


\subsection{Dependences}

Dependences were defined in Definition 7 as each different way of affecting or being affected by an entity. These dependences are not symmetric in a general case because they follow the cause-effect direction. For example, in the BBIs in Figure 3 and 4 the cause is $x$ and the effect $y$. Let us illustrate this concept of cause-effect direction by presenting a very basic BBI for measuring a temperature such that its signal $S$ depends on the temperature $T$ as $\quad S=0.01 T$.In this case, when the temperature changes, so does the signal following the relation $S=S(T)$. However, if the signal varies due to other reasons like noise or a bad calibration, the temperature is not going to change. Hence, even though the relation $T=100 \mathrm{~S}$ is mathematically correct, $T=T(S)$ is not a cause-effect dependence.

In a different scenario where the signal commands a heater with a feedback control loop, the two previous dependences can be present: the signal changes due to the temperature and, at the same time, this signal commands a control that changes the temperature.

For the purpose of this work, BBI dependences can be classified into three main blocks as detailed in the next points: stimuli-reactions, internal and complementary dependences.

\subsubsection{Stimuli-reactions dependences}

BBIs present a set of dependences between stimuli and reactions, linking them in such a way that when a stimuli affects the BBI, an adequate reaction emerges. These links represent dependences, called stimuli-reaction dependences. Examples can be found in Figures 3 and 4.

\subsubsection{Internal dependences}

BBIs contain on the inside a set of dependences which, based on the BBI definition (the constitution and structure of the box are altogether irrelevant to the approach under consideration), 
are not perceived from the outside. However, attending to Section 2, the evaluation of all dependences is a key aspect in the design process and consequently, these opaque dependences contained in the BBI cannot be neglected. The challenge is how to measure them when, from the outside, the knowledge about the internal BBI composition is very limited. This will be treated in Section 3.4.

\subsubsection{Complementary dependences}

Complementary dependences are the rest of the dependences contained in the product which are not stimuli-reaction nor internal dependences. For instance, the dependences created in the manufacturing or documentation process or the ones generated during maintenance activities and even during operation.

\subsection{Costs and dependences}

Section 3 has presented the different kind of dependences which can affect a BBI. They can be of a very different nature, what hampers the utilization of a universal measure for evaluating them. However, this can be solved thanks to the introduction of cost as a measure of dependences.

This link between cost and dependences was shown in Corollary 19 (Section 2.6.12). There, it is shown how reducing (increasing) the number and strength of dependences reduces (increases) the cost. Next two properties complement the previous ones showing that reducing (increasing) the cost reduces (increases) the number or strength of dependences.

Corollary 20: When output cost increases (decreases), the number or strength of dependences increases (decreases).

Proof of Corollary 20: When output cost changes there is a dependence which has been modified, added or deleted (since, by Definition 7, there is no other way to affect a product 
characteristic than through a dependence). Hence, a cost change means a dependence change. Since, as explained in the Corollary 19, "reducing (increasing) the number and strength of dependences reduces (increases) the output cost" there cannot be an output cost increase (reduction) and a dependence reduction (increase). Hence, the corollary holds.

Corollary 21: Output cost increases (decreases) precisely when the number or strength of dependences increases (decreases).

Proof of Corollary 21: It is a direct consequence of merging Corollary 19 and 20 into a single Corollary.

Hence, thanks to Corollary 21 , cost can be employed as a measure of dependences. It is important to remark that the cost employed for measuring these dependences should be a representative value, i.e., it shall not be altered by the inclusion of elements which will not likely exist in the future such as promotional offers or discounts. Costs are going to be split into two main groups:

1. Internal Costs: Costs incurred by the organization or stakeholders designing the product (e.g. manufacturing costs, verification costs and documentation costs). These costs have to be minimized during the design process for minimizing dependences as per Corollary 20 without impairing the satisfaction of NonDesigners Requirements.

2. External Costs: Costs incurred by the organizations or stakeholders not designing the product (e.g. operating costs and maintenance costs). They have to be considered in the design process through Non-Designers Requirements. Usually, they are going to be expressed as the conjunction of two Requirements: a) A Soft Requirement demanding minimum cost and b) a Hard Requirement setting a 
maximum cost. Nevertheless, there can be many other ways of expressing them depending on the concrete scenario.

The Soft Requirement related to the minimization of External Costs has to be addressed carefully by Designers since a reduction in a part of the External Costs can take with it an increase in other parts of the External Costs or in the Internal Costs. Even when these variations do not affect the output acceptance, they can significantly modify the satisfaction provided by it. In this section the concepts of acceptance and satisfaction are the same as the ones employed in Section 2, where acceptance is associated with the conformity (as per ISO 9000:2015) of HRs and satisfaction is associated to the perception of the degree to which SRs have been fulfilled. Hence, in this situation Designers have to evaluate the overall degree of satisfaction provided by each different Requirement for finally setting a concrete objective for each of the components of the External Costs.

As for Internal Costs, the different way how stimuli-reactions, internal and complementary dependences are treated can lead to diverse values, what will be detailed in the methodology of next section. Internal Costs contain, for example, the costs of acquiring the BBIS or the costs of assembling them.

For reducing Internal Costs, the number of BBIs contained in the output is an important element. The reason behind this statement is that each new BBI introduces a new dependence with, at least, a part of the output (otherwise this BBI could be removed). Consequently, this number shall be reduced. An indication of this can be observed when, with the addition of each new BBI, the costs of assembly, stock, documentation, etc. increase and so do dependences. 
The objective of the organization shall be to reduce as much as possible IC together with dependences, which go hand in hand, while satisfying Non-Designers Requirements. This will be treated in the methodology presented in next section.

\subsection{Methodology}

The objective of this section is to present a possible methodology to be followed during the design process, based on the results obtained in Section 2. For clarifying the nomenclature employed hereafter, the following definition is introduced:

Definition 18: An Available Output $(\mathrm{AvO})$ is a possible output conceived during the design process.

During the design process different AvOs can be considered for eventually selecting one among them, which will become the final output. Another definition of Available Outputs, which is aligned with the one of Definition 18, will be provided in Definition 21 (Section 4.2.2).

As explained in Section 2, the target of the design process is an output which minimizes the number of dependences and, at the same time, satisfies all Non-Designer Requirements. For this reason, the intention of the methodology is to reduce in a systematic way all dependences while preserving the fulfilment of the HRs. This last point is very important: Designers should always have present that, no matter how much they minimize dependences, if they do not satisfy Hard Non-Designers Requirements, then the output will not be accepted. For this reason, the fulfilment of Non-Designers Requirements has to be the foremost aspect to be considered by Designers.

In the end, the $\mathrm{AvO}$ to be selected shall present, in order of priority, the following three characteristics:

1. It satisfies Non-Designers Requirements. 
2. It minimizes stimuli-reactions dependences: Firstly stimuli-reactions dependences will be minimized through a process in which Designers decompose the AvO into BBIs for satisfying Non-Designers Requirements while minimizing the stimulireactions dependences. At the end of this process, BBIs will be defined by a set of Requirements they have to satisfy. This means that there are probably still some undetermined aspects of the $\mathrm{AvO}$ which depend on the concrete $\mathrm{BBI}$ selection, such as the value of certain variables. These pending aspects have to be defined minimizing the rest of dependences (i.e. internal and complementary dependences), using for it Internal Costs

3. It minimizes Internal Costs: As long as Internal Costs not only include the stimulireactions dependences (which have previously been minimized), but also internal and complementary dependences, this minimizations ensures an additional reduction of dependences.

Next points present the steps of the proposed methodology. Note that this methodology is not the only possible one which can put into practice the ideas of Section 2. However, this one was selected due to its staggered approach, the treatment of the dependences and the authors' experience:

\section{Step 1: Identify and evaluate the aspects which can affect the output acceptance and} verify the suitability of Requirements:

Identify and evaluate the aspects which can affect the output acceptance and hence, condition the design process. Examples are Non-Designers Requirements, elements from the work environment (defined as per ISO 9000:2015) or perturbations generated by uncertainty and variability which may condition the output acceptance. In addition, 
verify the suitability of the Requirements and solve any issue which can render the design process impossible (e.g. contradictory Requirements or the External Costs evaluation as explained in Section 3.4).

\section{Step 2: Determine the configuration of the output in terms of BBIs:}

Determine the configuration of the output in terms of other BBIs, linking them in such a way that BBIs provide the desired set of reactions as a consequence of a set of stimuli.

These BBIs are defined in terms of a set of Requirements. As remarked previously, reactions and stimuli shall always satisfy acceptance conditions, what can be facilitated by

- negotiating the reclassification into Soft Requirements of as many Hard Requirements as possible for preventing a rejection.

- loosening Requirements' acceptance conditions (e.g. widening acceptance intervals)

The $\mathrm{AvO}$ has to minimize the stimuli-reactions dependences of the BBIs and, at the same time, satisfy the corresponding acceptance conditions of Requirements. For example, there may be Requirements expressing the mere existence of a characteristic or an item which also has to be taken into account in this step. Examples are the colour of an item or the addition of an aesthetic element. The dependences established by elements such as those, which may not set stimuli-reactions dependences in a direct way are perceived through the internal and complementary dependences (to be evaluated in the next step). Indeed, in a general way the mere introduction of an element in the $\mathrm{AvO}$ already sets dependences, which can be measured by the cost of including this item in the $\mathrm{AvO}$ (i.e. internal dependences). Then, depending on the way how this 
element interacts with the rest of the AvO's components, additional dependences will arise.

It is important to note that the selection of a BBI may not be independent of the rest of BBIs which compose the output but it will usually be (at least) partially determined by them. Consequently, the order followed in the selection of the BBIs can have an important relevance in the final output. For this reason, Designers shall firstly determine the BBIs which set the strongest dependence, the highest number of dependences or have the highest cost. This is a key element of the methodology which Designers should always take into account.

As a tool for evaluating stimuli-reactions dependences, Designers can create the causeeffect matrix (Table 3) containing the dependences between the indicators, which represent the expected stimuli and reactions in and out of the BBIs $\left(I_{1}, I_{2}, I_{3} \ldots I_{\alpha}\right)$. The selection of these indicators is a decision of Designers. In principle it is recommended to include the ones of the Requirements and the ones which affect them. In any case, the whole set of dependences will be measured in the next steps by means of the costs. External Costs are not considered as a part of these indicators since they will be evaluated later on.

In the cause-effect matrix, causes are represented in rows and effects in columns. The matrix shall be filled out answering the following question: Does $I_{i}$ cause an effect in $I_{j}$ keeping the rest of the indicators constant (ceteris paribus)? If so, 1 is written in the intersection of the corresponding column and row. If not, 0 shall be written. In this way $\operatorname{dep}\left(\mathrm{I}_{\mathrm{i}}, \mathrm{I}_{\mathrm{j}}\right)$, representing the dependence between the cause $\mathrm{I}_{\mathrm{i}}$ and the effect $\mathrm{I}_{\mathrm{j}}$, acquires values in the following way: 
Does $I_{i}$ cause an effect in $I_{j}$ ceteris paribus? $\left\{\begin{array}{l}Y e s \rightarrow \operatorname{dep}\left(I_{i}, I_{j}\right)=1 \\ \operatorname{No} \rightarrow \operatorname{dep}\left(I_{i}, I_{j}\right)=0\end{array}\right.$

Normally, as it will be shown in the example of Section 3.6, the function given by Eq. 38 is enough to assess the importance of a dependence. However, if designers required a larger degree of detail, they could use the result obtained in Section 2.6.4 (see Eq. 29 after Theorem 4). Attending to that result, in order to assess the relative importance of a dependence, designers should answer the following questions: 'is sensitivity large, i.e. is $\partial f_{i}\left(I_{i, 0} ; \mathbf{n}\right) / \partial I_{i}$ large?', 'is the cause demanding, i.e. is $\bar{I}_{i}-\underline{I}_{i}$ large?', and 'is the effect demanding, i.e. is $\bar{I}_{j}-\underline{I}_{j}$ small?'. For example, if the answer is yes-yes-yes, the dependence is very strong. This larger degree of accuracy could be implemented by means of the application of Eqs. 38a to 38c to obtain an assessment such that: $0=$ no dependence, $1=$ normal dependence, $2=$ strong dependence and $3=$ very strong dependence.

Does $I_{i}$ cause an effect in $I_{j}$ ceteris paribus with

$$
\text { a large value of } \partial I_{j} / \partial I_{i} ?\left\{\begin{array}{l}
Y e s \rightarrow \operatorname{dep}\left(I_{i}, I_{j}\right)=1 \\
N o \rightarrow \operatorname{dep}\left(I_{i}, I_{j}\right)=0
\end{array}\right.
$$

Can be the required range of variation of $I_{i}$ very large?

$$
Y e s \rightarrow \operatorname{dep}\left(I_{i}, I_{j}\right)=\operatorname{dep}\left(I_{i}, I_{j}\right)+1
$$

Can be the required range of variation of $I_{j}$ very small?

$$
\text { Yes } \rightarrow \operatorname{dep}\left(I_{i}, I_{j}\right)=\operatorname{dep}\left(I_{i}, I_{j}\right)+1
$$

Note that this matrix is not symmetrical. In addition, since the dependence between an indicator and itself does not provide any relevant information, the values in the diagonal are removed. It is important to highlight that the higher the number of BBIs employed, 
the higher the likelihood of obtaining a large number of stimuli-reactions dependences.

The same happens if the number of stimuli and reactions is high.

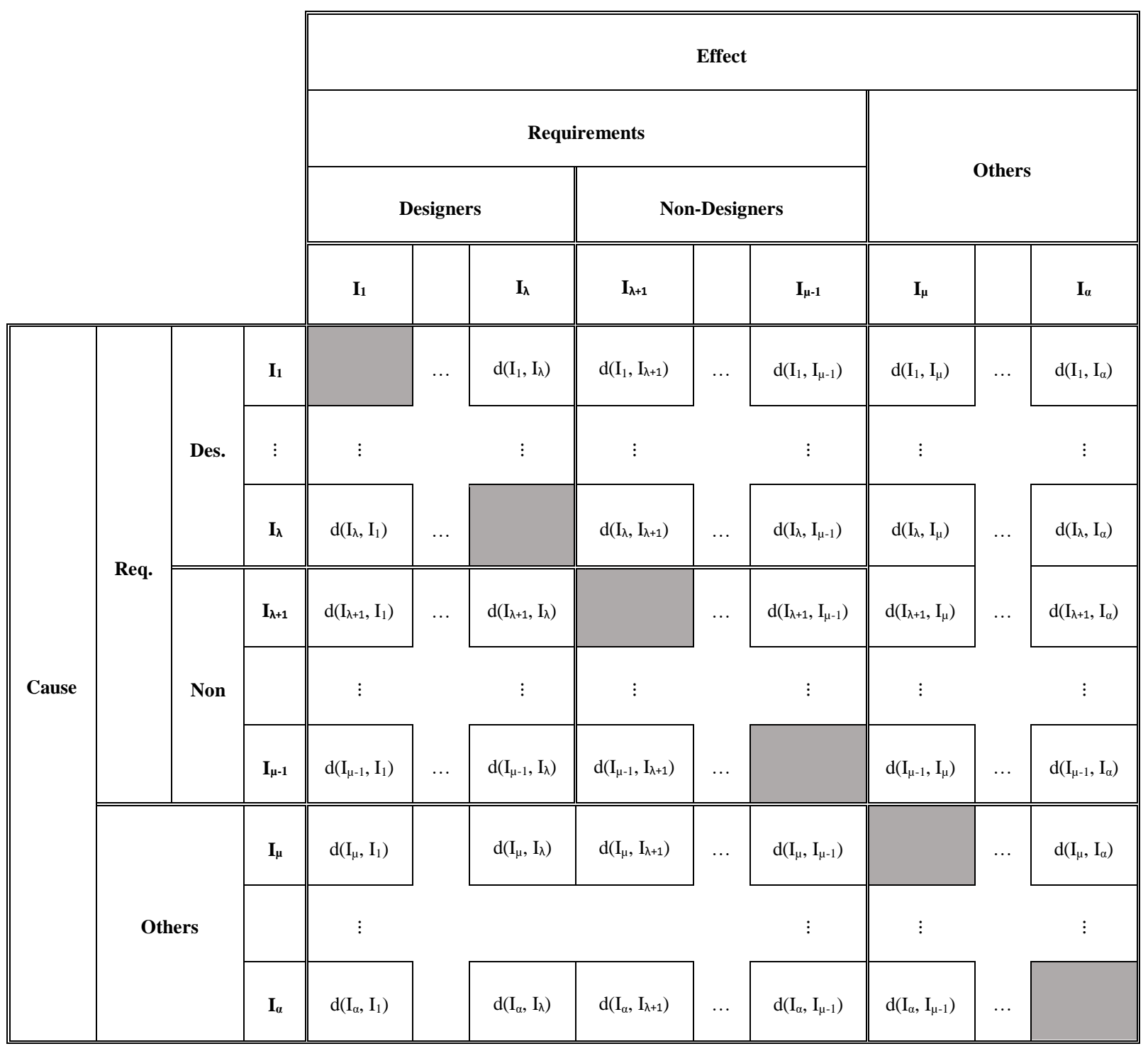

Table 3: Cause-effect matrix

Attending to the objective of reaching the minimum number of dependences, the causeeffect matrix should have all cells equal to zero except for the ones contained in the intersection of Cause/Requirements/Designers and Effect/Requirements/Non- 
Designers, which shall have only a single 1 in each cell and column (for both situations: Eq. 38 and Eq. 38a to 38c).

At the end of this step, $\mathrm{AvO}$ is defined in terms of the characteristics the different BBIs have to satisfy and it should minimize the dependences contained in cause-effect matrix while satisfying the corresponding Requirements.

Once step 2 is finished, there are usually a set of elements in the $\mathrm{AvO}$ still to be fixed which depend, among others, on the concrete BBIs selected. These undetermined elements of the AvO shall be chosen in such a way that the AvO Internal Costs are reduced and with it, dependences. This is the objective of next step 3, which evaluates dependences in terms of costs, as explained in Section 3.4.

\section{Step 3: Minimize Internal Costs:}

Step 2 has defined the AvO in terms of the characteristics that the different BBIs have to satisfy. Yet, there are usually several different BBIs which can satisfy those characteristics. The selection of one of them will entail not only the determination of a set of pending variables which can affect other BBIs but also additional dependences that will impact on Internal Costs. Hence, the objective of this step is to select these undetermined aspects of the $\mathrm{AvO}$ while minimizing Internal Costs and satisfying Requirements.

As in step 2, Designers shall firstly select the BBI which sets the highest number of dependences or has the highest cost. Then, the next one and so on. In this process, the different BBIs are chosen minimizing IC. During this minimization, the selection of BBIs with a low cost is an important task, even though this aspect is not enough for 
minimizing Internal Costs if the rest of the costs are not also considered. To this end, the following two parameters are relevant:

- Number of substitutive BBIs (\#SB): Substitutive BBIs are the set of available BBIs which fulfil the same set of Requirements. An increase in the number of substitutive BBIs lowers the dependences on the initial number of BBIs. An example which can be indicative of this phenomenon is that, if a BBI has many alternatives, its price tends to be lower than a BBI with a few alternatives: finding a replacement for a specific part which is no longer manufactured can be hard and expensive. In the same way, the possibility of benefiting from different offers due to market competition is higher when more substitutive BBIs are present. Consequently, the higher the number of substitutive BBIs, the better.

- Number of Available Suppliers (\#AS): The existence of different suppliers lowers the dependences on a concrete supplier or set of them. For instance, the existence of a single or few suppliers in a market usually gives rise to higher prices. This can also have a relation with monopolies, oligopolies and other markets forms which are usually considered to lead to higher prices for consumers.

This step is finished when all BBIs have been determined minimizing Internal Costs and hence, Designers have a complete description of the output, removing in this way the undetermined elements resulting from step 2. 


\section{Step 4: Evaluate External Costs Requirements:}

Verify whether the External Costs Requirements are satisfied or not. If not, revisit the Internal Costs process (step 3) or redesign the output configuration (step 2). This step may require the inclusion of extra dependences and hence, to increase the minimum number obtained previously.

\subsection{Example}

The objective of this section is to present a practical example of the previous methodology. In this example, a company detects the need for a high-precision electrical heater during the definition of a thermal conductance test between two parts in a vacuum chamber. This starts off a design process which will be detailed in the next steps, numbered in accordance to the ones presented in Section 3.5.

\section{Step 1: Identify and evaluate the aspects which can affect the output acceptance and}

\section{verify the suitability of Requirements:}

In this step Designers have to identify the aspects which can affect the output acceptance and hence, condition the design process. The first aspect they are going to determine is Non-Designers Requirements. To this end, the customer complements the initial information indicating that the heater shall provide a power of $10 \mathrm{~W} \pm 2 \%$ with an acquistion cost under $150 €$. Based on this, Designers define the following Non-Designers Requirements:

- Input Requirement: Electrical

- Heat Requirement: $Q \in[9.8 \mathrm{~W}, 10.2 \mathrm{~W}]$

- $1^{\text {st }}$ External Costs Requirement: Minimum acquisition cost

- $2^{\text {nd }}$ External Costs Requirement: Acquisition cost $<150 €$ 
Input, Heat and $2^{\text {nd }}$ External Costs Requirements are Hard Requirements whereas $1^{\text {st }}$ External Costs Requirement is Soft. Once these Requirements have been determined, Designers verify the suitability of the Requirements and solve any issue which can render the design process impossible. Consequently, the first important task is to verify that the four Requirements are compatible. In this case each one addresses a different concern and does not overlap with the others, being thus compatible each one with the others. The second important task is the treatment of the External Costs Requirements. The $1^{\text {st }}$ External Costs Requirement can be left as it is since the minimum acquisition cost is related to the Internal Costs minimization, which will be an objective of the design process. The $2^{\text {nd }}$ External Costs Requirement can be expressed in an alternative way considering that Designers want to obtain a $20 \%$ profit in the transaction. This means that Internal Costs have to be under $120 €$ and consequently, the $2^{\text {nd }}$ External Costs Requirement can be reformulated as IC $<120 €$. Thanks to this, Designers know that no matter what kind of design philosophy they follow, if IC $>120 €$, the AvO is amiss.

Yet, there are still other aspects which can affect the output acceptance and have to be considered by Designers. The first one is the voltage of the electrical grid to which the heater is going to be connected. Taking into account that the premises of the company are located in Europe, Designers know they have to design the heater for a grid supply equal to $230 \mathrm{~V} \pm 10 \%$ (CENELEC HD 472 S1, 1988). This means they will have to deal with the incoming voltage variations. Nevertheless, this is not the only variation which will have a relevant impact on the design process but also the effect of temperature on the different components, especially on the resistance value. Designers carry out a preliminary assessment of these variations starting from the equation which relates power to voltage and the circuit resistance:

$$
Q=V^{2} / R
$$


If $\varepsilon_{v}$ represents the variations coming from the electrical grid and $\varepsilon_{r}$ represent the variations resulting from temperature changes such that $V=\widehat{V}\left(1+\varepsilon_{V}\right)$ and $R=\widehat{R}\left(1+\varepsilon_{R}\right)$, they generate a variation in heat, $\varepsilon_{Q}$, such that $Q=\widehat{Q}\left(1+\varepsilon_{Q}\right)$, which satisfy the following relation:

$$
\hat{Q}\left(1+\varepsilon_{Q}\right)=\frac{V^{2}\left(1+\varepsilon_{V}\right)^{2}}{\hat{R}\left(1+\varepsilon_{R}\right)} \cong \frac{\hat{V}^{2}}{\hat{R}}\left(1+2 \varepsilon_{V}-\varepsilon_{R}\right)
$$

and thus

$$
\varepsilon_{Q}=2 \varepsilon_{V}-\varepsilon_{R}
$$

However, since variations can be indistinctively positive or negative, Eq. 41 is reformulated as

$$
\left|\varepsilon_{Q}\right|=2\left|\varepsilon_{V}\right|+\left|\varepsilon_{R}\right|
$$

In this case, there is a critical factor which has to be corrected for satisfying the Heat Requirement: voltage induces variations of $10 \%$ whereas the maximum variation allowed in the Heat Requirement is $2 \%$. Indeed, as Equation 42 shows

$$
0.02>0.2+\left|\varepsilon_{r}\right|
$$

Eq. 43 is impossible to satisfy, no matter how small $\boldsymbol{\varepsilon}_{r}$ is. Therefore, this analysis has determined that Designers need to reduce the electrical grid variations in their design process for satisfying Non-Designers Requirements.

\section{Step 2: Determine the configuration of the output in terms of BBIs:}

In this step Designers have to determine the configuration of the output in terms of other BBIs linking them in such a way that BBIs provide the desired set of reactions as a consequence of a set of stimuli. In addition, the $\mathrm{AvO}$ has to minimize the stimuli-reactions dependences of the BBIs and, at the same time, satisfy the corresponding acceptance conditions of Requirements 
To this end, Designers come up with four different AvOs which satisfy the Requirements but only one minimizes stimuli-reactions dependences. All AvOs incorporate a Power Supply (PS) for satisfying the Input Requirement in the presence of excessive power grid oscillations.

\section{AvO 1:}

The first $\mathrm{AvO}$ considered is a resistor connected to a PS as detailed in Figure 5. In this AvO, PS is out of the vacuum chamber and thus, temperature oscillations induce negligible changes in its performance. The resistor is the component in charge of generating the required heat, located inside the vacuum chamber. For this reason, it will be subjected to relevant temperature fluctuations which can impair its performance.

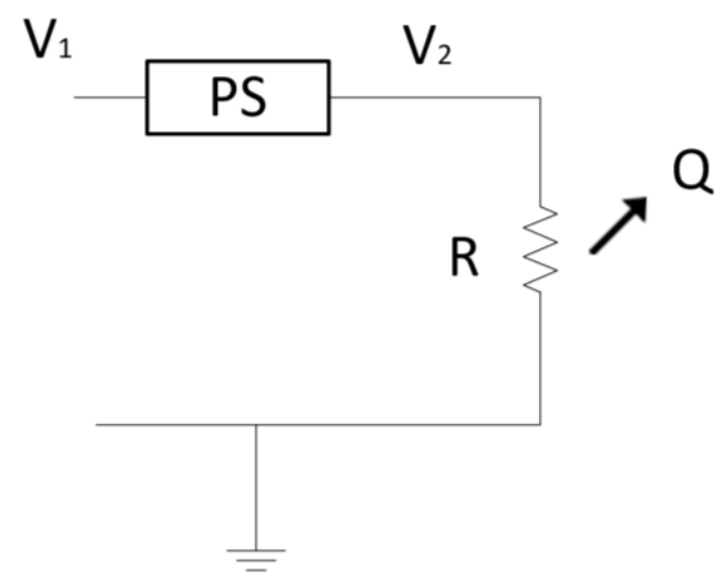

Figure 5: AvO 1

The indicators of this AvO, partially represented in Figure 5, are the following: 


\begin{tabular}{|c|l|}
\hline Indicator & \multicolumn{1}{|c|}{ Variable } \\
\hline $\mathrm{V}_{1}$ & Electrical grid voltage \\
\hline $\mathrm{V}_{2}$ & Voltage out of the power supply \\
\hline $\mathrm{Q}$ & Heat (indicator of the Heat Requirement) \\
\hline $\mathrm{R}$ & Resistance \\
\hline $\mathrm{T}_{\mathrm{c}}$ & Temperature of the parts in the vacuum chamber \\
\hline
\end{tabular}

Table 4: AvO 1 indicators

$\mathrm{Q}$ is a Non-Designer Indicator whereas $\mathrm{V}_{2}$ and $\mathrm{R}$ stem from Designers decisions, and hence, they are Designers Indicators. $V_{1}$ is set by the electrical grid provider whereas $T_{c}$ and $T_{o}$ appear due to environmental effects. Consequently, they cannot be associated to any Requirement. Based on these indicators, the cause-effect matrix is presented in Table 5, which contains 9 dependences. There are two dependences in the intersection of Cause/Requirements/ Designers with Effect/Requirements/Non-Designers being the ideal case just one. 


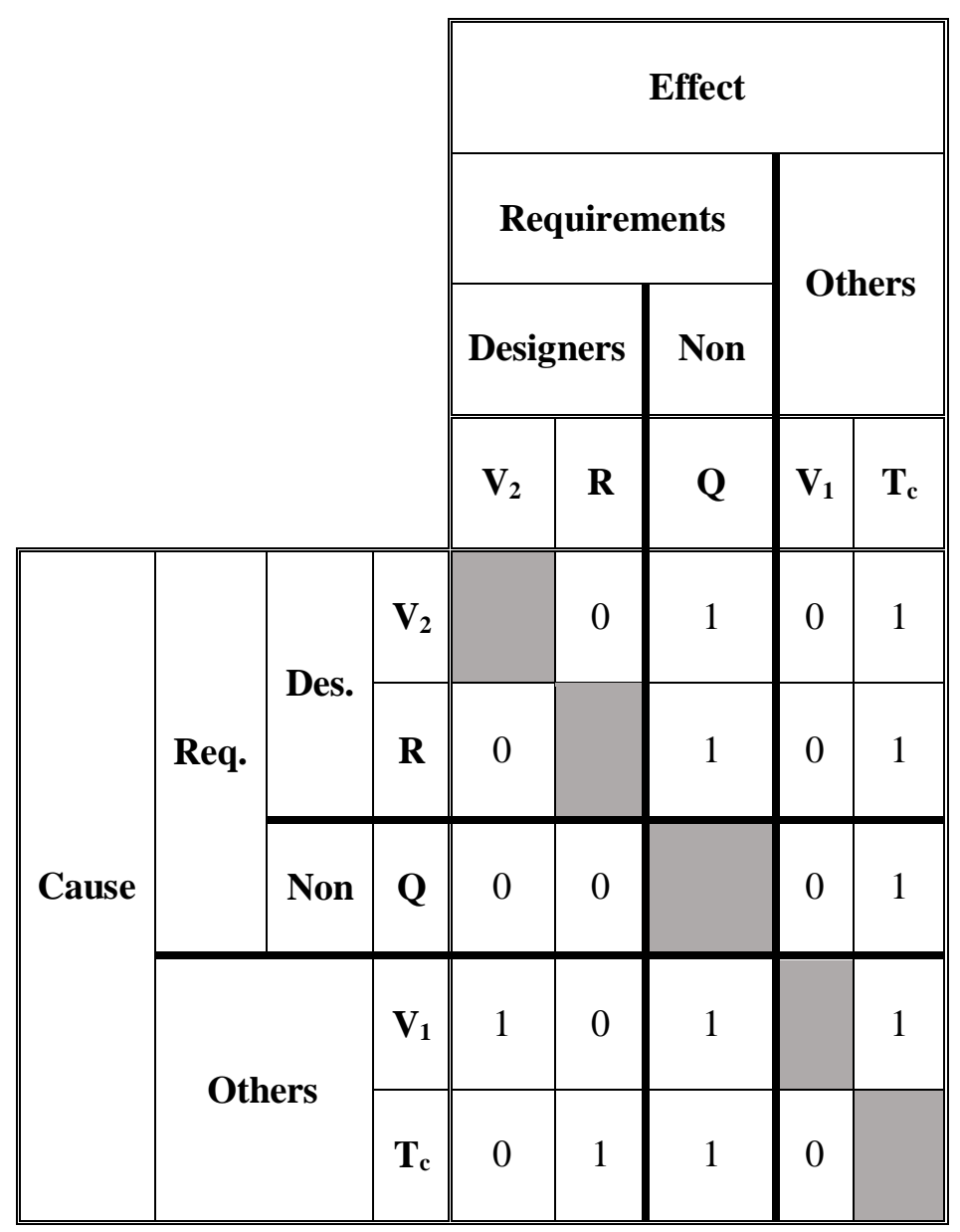

Table 5: Cause-effect matrix of AvO 1

The dependence between $\mathrm{T}_{\mathrm{c}}$ and $\mathrm{R}$ (and thus $\mathrm{Q}$ ) is one of the main drawbacks of this design. Resistance, critical for determining the heat dissipated, depends on temperature, which varies significantly in the place where the resistor is located (specially taking into account that the objective of the device is to heat up a material). This will likely lead to the selection of a highprecision resistor in the next steps of the design process, whose price will be higher and its availability lower. Even though this $\mathrm{AvO}$ is the most appropriate one in terms of stimuli-reactions dependences, three additional AvOs are presented for further exemplifying the methodology. 


\section{AvO 2:}

AvO 2 employs a MOSFET for dissipating the required heat (Figure 6). This MOSFET is placed inside the vacuum chamber and its resistance is set through $\mathrm{V}_{\text {ref. }}$ The device in charge of generating $\mathrm{V}_{\text {ref }}$ as well as PS are out of the vacuum chamber and their performance is assumed to have a negligible variation with $\mathrm{T}_{\mathrm{o}}$.

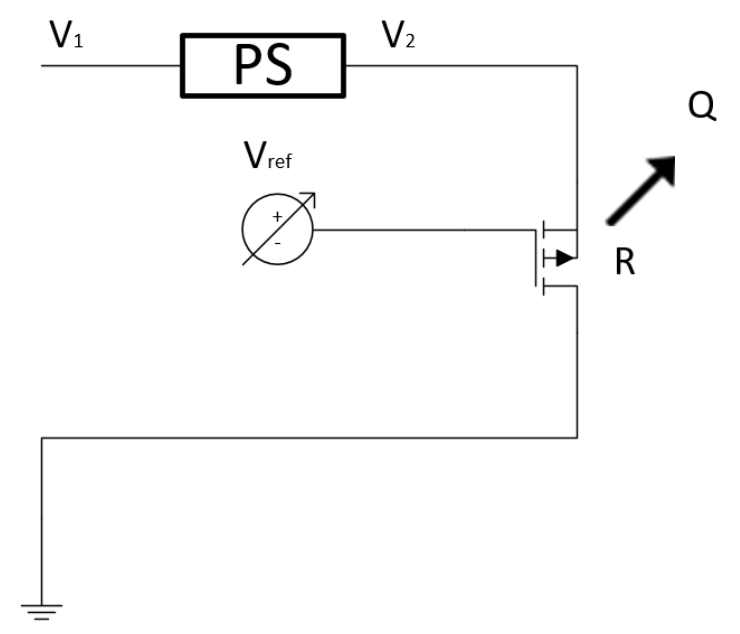

Figure 6: $\mathrm{AvO} 2$

This $\mathrm{AvO}$ requires knowing the relation between $\mathrm{V}_{\text {ref }}$ and the intensity going through the MOSFET. In a more detailed stage of the design, this will lead to the selection of a high precision MOSFET, which will make the output more expensive. The indicators of this AvO are the following: 


\begin{tabular}{|c|l|}
\hline Indicator & \multicolumn{1}{|c|}{ Variable } \\
\hline $\mathrm{V}_{1}$ & Electrical grid voltage \\
\hline $\mathrm{V}_{2}$ & Voltage out of the power supply \\
\hline $\mathrm{V}_{\text {ref }}$ & Reference voltage \\
\hline $\mathrm{Q}$ & Heat (indicator of the Heat Requirement) \\
\hline $\mathrm{R}$ & Resistance \\
\hline $\mathrm{T}_{\mathrm{c}}$ & Temperature of the parts in the vacuum chamber \\
\hline
\end{tabular}

Table 6: AvO 2 indicators

Based on these indicator, the cause-effect matrix is obtained in next Table 7, which shows 13 dependences. In addition, the intersection of Cause/Requirements/Designers with Effect/Requirements/Non-Designers shows three dependences. These are the reasons why, when both AvOs 1 and 2 satisfy Non-Designers Requirements, AvO 2 is worse than $\mathrm{AvO} 1$. 


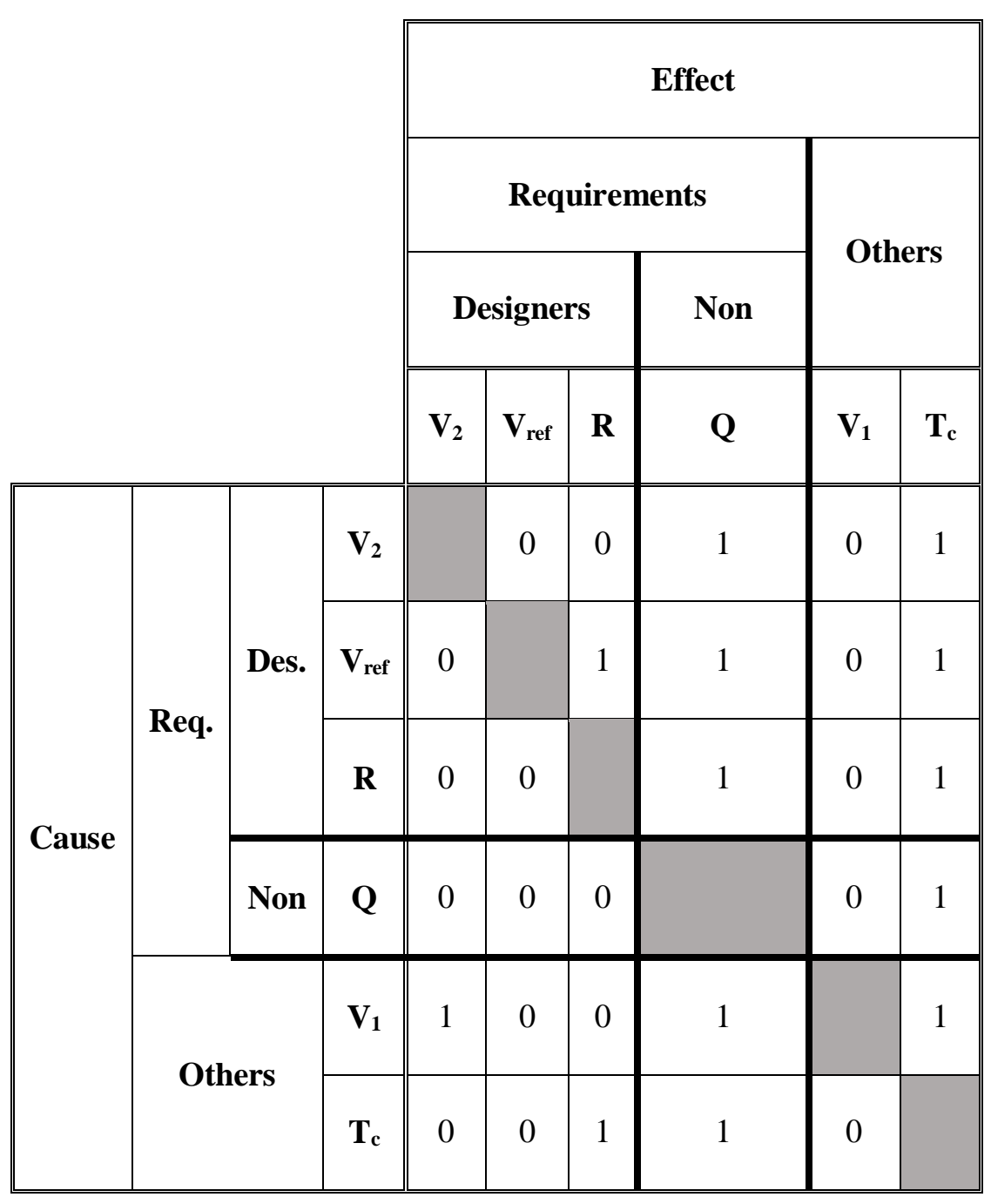

Table 7: Cause-effect matrix of AvO 2

Table 7 shows, as in $\mathrm{AvO} 1$, a dependence between the temperature fluctuation in the vacuum chamber and the variation of the resistance.

\section{AvO 3:}

AvO 3 incorporates a MOSFET as in $\mathrm{AvO} 2$. However, in this case an operational amplifier and an additional resistor $\mathrm{R}_{2}$ are also included. The operational amplifier works trying to keep the difference of its input voltage as close to zero as possible. Thus, the voltage over $\mathrm{R}_{2}$ will be kept 
at $\mathrm{V}_{\text {ref. }}$. All the elements will be located out of the vacuum chamber except for the MOSFET, which will be inside.

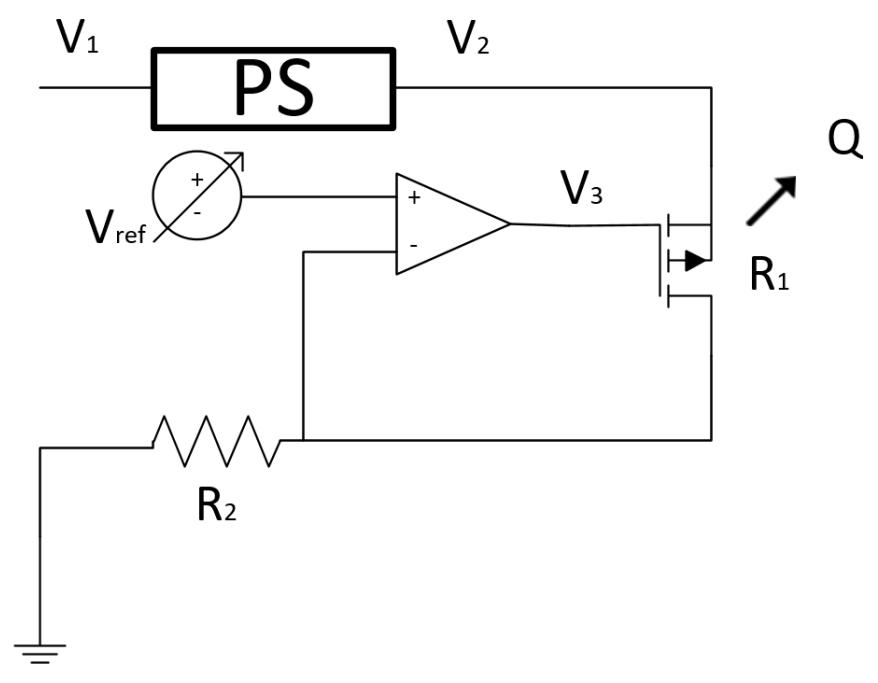

Figure 7: AvO 3

Due to $V_{\text {ref }}$ and the op-amp, the intensity going through the MOSFET satisfies the following equality:

$$
I=V_{\text {ref }} / R_{2}
$$

Based on this, the heat dissipated in the MOSFET will be equal to

$$
Q=\left(V_{2}-V_{\text {ref }}\right) I=\left(V_{2}-V_{\text {ref }}\right) V_{\text {ref }} / R_{2}
$$

Eq. 45 shows that the heat generated does not depend on the MOSFET variables. Even though this MOSFET will suffer the effect of the temperature changes (as the resistor in AvOs 1 and 2), the op-amp corrects them subsequently.

However, there is still a key indicator which sets a dependence with temperature: $\mathrm{R}_{2}$, The upside is that the environment where it is placed is not as thermally aggressive as the one in $\mathrm{AvO}$ 1 , and hence these variations are significantly lower. The indicators of this design are detailed in next Table 8. 


\begin{tabular}{|c|l|}
\hline Indicator & \multicolumn{1}{|c|}{ Variable } \\
\hline $\mathrm{V}_{1}$ & Electrical grid voltage \\
\hline $\mathrm{V}_{2}$ & Voltage out of the power supply \\
\hline $\mathrm{V}_{3}$ & Voltage out of the op-amp \\
\hline $\mathrm{V}_{\mathrm{ref}}$ & Reference voltage \\
\hline $\mathrm{Q}$ & Heat (indicator of the Heat Requirement) \\
\hline $\mathrm{R}_{1}$ & Dissipative Resistance \\
\hline $\mathrm{R}_{2}$ & Control Resistance \\
\hline $\mathrm{T}_{\mathrm{c}}$ & Temperature of the parts in the vacuum chamber \\
\hline $\mathrm{T}_{\mathrm{o}}$ & Temperature of the parts out of the vacuum chamber \\
\hline
\end{tabular}

Table 8: AvO 3 indicators

Based on these indicator, the cause-effect matrix is created (Table 9), which shows 22 dependences, many more than the 9 dependences of $\mathrm{AvO} 1$ and the 12 dependences of $\mathrm{AvO} 2$. This increase in the number of dependences arises as a consequence of complicating the design for removing the dependence of resistance with temperature, present in AvOs 1 and 2. However, the removal of this dependence and the subsequent increment in the number of dependences is not necessary as long as $\mathrm{AvO} 1$ is able to cope with this dependence while satisfying Non-Designer Requirements. In a different scenario where Designers knew that neither AvO 1 nor $\mathrm{AvO} 2$ is able 
to satisfy Non-Designers Requirements due to this dependence, AvO 3 would emerge as a potential alternative.

\begin{tabular}{|c|c|c|c|c|c|c|c|c|c|c|c|c|}
\hline & & & & \multicolumn{9}{|c|}{ Effect } \\
\hline & & & & \multicolumn{6}{|c|}{ Requirements } & \multirow{2}{*}{\multicolumn{3}{|c|}{ Others }} \\
\hline & & & & \multicolumn{5}{|c|}{ Designers } & \multirow{2}{*}{$\begin{array}{c}\text { Non } \\
\mathbf{Q}\end{array}$} & & & \\
\hline & & & & $\mathbf{V}_{2}$ & $\mathbf{V}_{3}$ & $\mathbf{V}_{\text {ref }}$ & $\mathbf{R}_{1}$ & $\mathbf{R}_{2}$ & & $V_{1}$ & $\mathbf{T}_{\mathrm{c}}$ & $\mathbf{T}_{\mathbf{0}}$ \\
\hline \multirow{9}{*}{ Cause } & \multirow{6}{*}{ Req. } & \multirow{5}{*}{ Des. } & $\mathbf{V}_{2}$ & & 1 & 0 & 0 & 0 & 1 & 0 & 1 & 0 \\
\hline & & & $\mathbf{V}_{3}$ & 0 & . & 0 & 1 & 0 & 1 & 0 & 1 & 0 \\
\hline & & & $\mathbf{V}_{\text {ref }}$ & 0 & 1 & & 1 & 0 & 1 & 0 & 1 & 0 \\
\hline & & & $\mathbf{R}_{1}$ & 0 & 1 & 0 & & 0 & 0 & 0 & 0 & 0 \\
\hline & & & $\mathbf{R}_{\mathbf{2}}$ & 0 & 1 & 0 & 1 & & 1 & 0 & 1 & 0 \\
\hline & & Non & $\mathbf{Q}$ & 0 & 0 & 0 & 0 & 0 & & 0 & 1 & 0 \\
\hline & \multirow{3}{*}{\multicolumn{2}{|c|}{ Others }} & $\mathbf{V}_{1}$ & 1 & 1 & 0 & 0 & 0 & 1 & & 1 & 0 \\
\hline & & & $\mathbf{T}_{\mathbf{c}}$ & 0 & 0 & 0 & 1 & 0 & 0 & 0 & & 0 \\
\hline & & & $\mathbf{T}_{\mathbf{0}}$ & 0 & 0 & 0 & 0 & 1 & 0 & 0 & 0 & \\
\hline
\end{tabular}

Table 9: Cause-effect matrix of AvO 3 


\section{AvO 4:}

Figure 8 shows $\mathrm{AvO} 4$ where, again, a MOSFET is employed for dissipating heat. In this case, the feedback loop for dealing with the temperature-induced variations is kept but, instead of using a resistor for measuring the current as in AvO 3, a coil with a Hall Effect Sensor (HES) is employed, providing a voltage correlated to the current passing through it. Hence, the voltage applied to the negative entry of the op-amp is linked to the current going through the coil (and thus, through the MOSFET). As in the previous AvOs, the only component which will be inside the vacuum chamber is the MOSFET. The rest will be on the outside and hence, even though HES devices are also subjected to thermal variations due to temperature oscillations, these variations will be significantly smaller than on the inside.

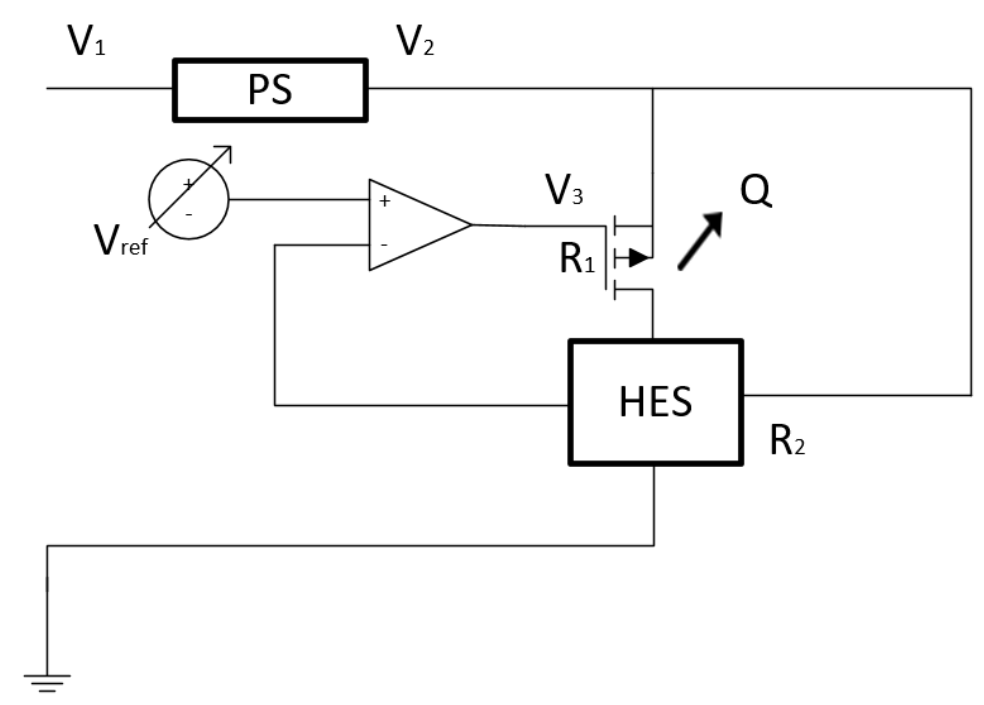

Figure 8: $\mathrm{AvO} 4$

The indicators of this $\mathrm{AvO} 4$ are detailed in next Table 10. 


\begin{tabular}{|c|l|}
\hline Indicator & \multicolumn{1}{|c|}{ Variable } \\
\hline $\mathrm{V}_{1}$ & Electrical grid voltage \\
\hline $\mathrm{V}_{2}$ & Voltage out of the power supply \\
\hline $\mathrm{V}_{3}$ & Voltage out of the op-amp \\
\hline $\mathrm{V}_{\mathrm{ref}}$ & Reference voltage \\
\hline $\mathrm{Q}$ & Heat (indicator of the Heat Requirement) \\
\hline $\mathrm{R}_{1}$ & Dissipative Resistance \\
\hline $\mathrm{R}_{2}$ & HES Resistance \\
\hline $\mathrm{T}_{\mathrm{c}}$ & Temperature of the parts in the vacuum chamber \\
\hline $\mathrm{T}_{\mathrm{o}}$ & Temperature of the parts out of the vacuum chamber \\
\hline
\end{tabular}

Table 10: $\mathrm{AvO} 4$ indicators

Based on these indicator, the cause-effect matrix can be created, which shows 24 dependences. As explained in $\mathrm{AvO} 3$, this increment in the number of dependences for breaking other dependences is only justifiable if the AvOs which present a lower number of dependences cannot satisfy Non-Designers Requirements. 


\begin{tabular}{|c|c|c|c|c|c|c|c|c|c|c|c|c|}
\hline & & & & \multicolumn{9}{|c|}{ Effect } \\
\hline & & & & \multicolumn{7}{|c|}{ Requirements } & \multirow{2}{*}{\multicolumn{2}{|c|}{ Others }} \\
\hline & & & & \multicolumn{6}{|c|}{ Designers } & Non & & \\
\hline & & & & $\mathbf{V}_{2}$ & $\mathbf{V}_{3}$ & $\mathbf{V}_{\text {ref }}$ & $\mathbf{R}_{1}$ & $\mathbf{R}_{2}$ & $\mathbf{Q}$ & $\mathbf{V}_{1}$ & $\mathbf{T}_{\mathbf{c}}$ & $\mathbf{T}_{\mathbf{0}}$ \\
\hline \multirow{9}{*}{ Cause } & \multirow{6}{*}{ Req. } & \multirow{5}{*}{ Des. } & $\mathbf{V}_{2}$ & & 1 & 0 & 0 & 0 & 1 & 0 & 1 & 0 \\
\hline & & & $V_{3}$ & 0 & . & 0 & 1 & 0 & 1 & 0 & 1 & 0 \\
\hline & & & $\mathbf{V}_{\text {ref }}$ & 0 & 1 & . & 1 & 0 & 1 & 0 & 1 & 0 \\
\hline & & & $\mathbf{R}_{1}$ & 0 & 1 & 0 & & 0 & 1 & 0 & 1 & 0 \\
\hline & & & $\mathbf{R}_{2}$ & 0 & 1 & 0 & 1 & & 1 & 0 & 1 & 0 \\
\hline & & Non & $\mathbf{Q}$ & 0 & 0 & 0 & 0 & 0 & & 0 & 1 & 0 \\
\hline & \multirow{3}{*}{\multicolumn{2}{|c|}{ Others }} & $\mathbf{V}_{1}$ & 1 & 1 & 0 & 0 & 0 & 1 & & 1 & 0 \\
\hline & & & $\mathbf{T}_{\mathbf{c}}$ & 0 & 0 & 0 & 1 & 0 & 0 & 0 & & 0 \\
\hline & & & $\mathbf{T}_{\mathbf{0}}$ & 0 & 0 & 0 & 0 & 1 & 0 & 0 & 0 & \\
\hline
\end{tabular}

Table 11: Cause-effect matrix of $\mathrm{AvO} 4$ 


\section{Step 3: $\quad$ Minimize Internal Costs:}

The objective of this step is to select these undetermined aspects of the $\mathrm{AvO}$ while minimizing Internal Costs and satisfying the Requirements. To this end, firstly the BBIS which set the highest number of dependences or have the highest cost are evaluated. In this case there are two BBIs which set the highest number of stimuli-reactions dependences in AvO 1: PS and the resistor (i.e. 3 dependences as per Table 5). Eventually, the PS is selected as the first BBI to be completely determined since Designers estimate that it will have higher internal dependences than the resistor (and higher cost).

In the process of the full PS determination, Designers need to set another Requirement: maximum $\mathrm{V}_{2}$ oscillation. This decision is made based on a previous evaluation of tolerances allocation between the resistor and the PS. Taking into account that heat can stand a $\pm 2 \%$ variation according to the Heat Requirement, Designers decide that $\mathrm{V}_{2}$ variation shall be equal or smaller than $\pm 0.5 \%$. This Requirements adds to the one about the input voltage (i.e. $V_{1} \in[207 \mathrm{~V}, 253 \mathrm{~V}]$ ) and power (i.e. > $10 \mathrm{~W}$ ). Designers initially classify their alternatives into two main blocks: Embedded Switch Mode Power Supplies (ESMPS) and Embedded Linear Power Supplies (ELPS). Then, they evaluate \#SB, \#AS and the lowest BBI cost among the alternatives.

\begin{tabular}{|l|c|c|c|}
\cline { 2 - 4 } \multicolumn{1}{c|}{} & \multicolumn{3}{c|}{ Power Supply } \\
\cline { 2 - 4 } \multicolumn{1}{c|}{} & \#SB & \#AS & Lowest BBI Cost \\
\hline \hline ESMPS & 523 & 3 & $45 €$ \\
\hline ELPS & 51 & 1 & $65 €$ \\
\hline
\end{tabular}

Table 12: Power Supply \#SB, \#AS and lowest BBI cost 
Table 12 shows how ESMPS have higher \#SB and \#AS and lower BBI cost among the alternatives. Taking into account that the impact of the two options on the rest of the cost is pretty similar, Designers decide to select the ESMP option. The next decision is to select the output voltage, or in other words, the value of $\mathrm{V}_{2}$. To this end, Table 13 presents the classification of the different voltages based on \#SB, \#AS and the lowest BBI cost.

\begin{tabular}{|l|c|c|c|}
\cline { 2 - 4 } \multicolumn{1}{c|}{} & \multicolumn{3}{c|}{ Power Supply V } \\
\cline { 2 - 4 } \multicolumn{1}{c|}{} & \#SB & \#AS & Lowest BBI Cost \\
\hline \hline ESMPS 3.3 V & 7 & 1 & $60 €$ \\
\hline ESMPS 5 V & 95 & 2 & $60 €$ \\
\hline ESMPS 12 V & 131 & 2 & $45 €$ \\
\hline ESMPS 15 V & 71 & 3 & $50 €$ \\
\hline ESMPS 24 V & 94 & 1 & $50 €$ \\
\hline ESMPS 36 V & 16 & 1 & $110 €$ \\
\hline ESMPS 48 V & 39 & 2 & $100 €$ \\
\hline
\end{tabular}

Table 13: Power supply voltage \#SB, \#AS and the lowest BBI cost

Based on the results of Table 13, a $12 \mathrm{~V}$ power supply is chosen, which has the lowest cost among the alternatives and the highest values of \#SB and \#AS.

Now, it is time to set the value of the resistor which has to be such that $R \in[15.2 \Omega, 15.5 \Omega]$ (i.e. $15.35 \Omega \pm 0.977 \%$ ). Taking into account this Requirement, Designers find a fixed resistor of $15.4 \Omega \pm 0.1 \%$ which lies inside the previous interval and has the following characteristics: 


\begin{tabular}{|c|c|c|c|}
\cline { 2 - 4 } \multicolumn{1}{c|}{} & \multicolumn{3}{c|}{ Resistance } \\
\cline { 2 - 4 } \multicolumn{1}{c|}{} & \#SB & \#AS & Lowest BBI Cost \\
\hline $15.4 \Omega \pm 0.1 \%$ & 2 & 1 & $0.36 €$ \\
\hline
\end{tabular}

Table 14: Resistor \#SB, \#AS and lowest BBI cost

The previous BBIs have to be complemented with other BBIs which had not been explicitly named in the AvO description: wires, screws, case, etc. The cost of these elements together with the resistor and PS are represented in next Table 15.

\begin{tabular}{|l|c|}
\cline { 2 - 2 } \multicolumn{1}{c|}{} & Lowest BBIs cost \\
\hline \hline Resistor & $5.02 €$ \\
\hline Power Supply & $45.00 €$ \\
\hline Other (wires, screws...) & $7.25 €$ \\
\hline & $\mathbf{5 7 . 2 7} €$ \\
\hline
\end{tabular}

Table 15: AvO 1 lowest BBI cost

Table 15 shows that the total cost of all BBIs in AvO 1 is equal to $57.27 €$. However, the previous costs are not the only ones. Designers also have to estimate the cost of the other aspects, such as documentation, assembly process, etc... These costs will depend on many internal aspects of the company and they are going to be called Additional Costs (AC). With this, the IC is calculated as

$$
I C=57.27 €+A C
$$




\section{Step 4: $\quad$ Evaluate External Costs Requirements:}

Eventually, in this step Designers verify whether the External Costs Requirements are satisfied or not. As explained previously, taking into account that Designers want to obtain a $20 \%$ profit in the transaction and that the customer is not willing to pay more than $150 €$, Internal Costs have to be under $120 €$, what will be satisfied if and only if $\mathrm{AC}<62.73 €$ according to Eq. 46 .

\subsection{Conclusions}

The main objective of this study has been to present a methodology for minimizing dependences during the design process. To this end, the identification of the basic bricks employed by Designers for the design process has been prime, which have been called Black Box Items. These are items whose constitution and structure are altogether irrelevant, being the important characteristic what happens at their interfaces. Linked to the Black Box Items, there are a set of dependences. Firstly the ones present at their interfaces, called stimuli-reactions dependences and then their innate dependences, called internal dependences. In addition, during the design process other dependences will arise, called complementary dependences. All of them will be minimized during the methodology.

A relevant aspect is that dependences can be of a very different nature what makes difficult to find a global measure for evaluating them. In order to solve this aspect, a correlation is found between cost and dependences, adopting the former as a measure of the latter.

Based on these concepts, a methodology is presented which defines the output in terms of Black Box Items. This methodology minimizes firstly stimuli-reactions dependences through the cause-effect matrix and then, the rest of dependences through a cost minimization. Thanks to this process, eventually an output with minimum dependences is obtained. 


\section{THE IDEAL DESIGN PROCESS}

\subsection{Introduction}

The section analyses how preliminary decision-making tools such as the one presented in Sections 2 and 3 can affect the design process. To this end, firstly a set of definitions and properties are presented in order to characterize the Ideal Design Process, defined as a rule-based design process which obtains the Best Output for a given set of Requirements. Rule-based means that the outcome is deterministic or, in other words, for a given input, there is a single output This Ideal Design Process, despite being rule-based, will have an innate stochastic nature. This fact will lead to a stochastic behavior of the two resources consumed by it: time and money.

Then, two different design configurations are presented. The first configuration is a design process composed of two phases: a synthesis phase, which includes the creativity required for innovation, and a very powerful detailed-analysis phase, which uses the maximum number of decision criteria for selecting the best output. The second configuration also includes a nondetailed (or preliminary) analysis phase ahead of the detailed-analysis process, which represents a preliminary decision-making tool rejecting the solutions which do not satisfy a set of conditions. The solutions which satisfy these conditions are then evaluated by the analysis process. In order to compare these two configurations, the resources invested during these design phases are computed in a general case. The proposed model allows the study of many different design scenarios by setting the corresponding times and expenses associated to the different phases of the design process.

In the next step, the two configurations are applied to a concrete case: the evaluation of the impact of preliminary decision-making tools on the design process. It is assumed the design 
process improves when the best output is obtained with a lower consumption of resources and with a lower uncertainty and variability, both concepts related to acquired complexity and risk. Thanks to this, the conditions that a preliminary decision-making tool needs to satisfy to improve the design process are obtained: compatible, fast, inexpensive, absolute, reusable and very selective. Design scenarios where the preliminary decision-making tool deteriorates the design process are also found. Eventually, Axiomatic Design is studied under this framework, showing evidences that indicate the satisfaction of this set of properties.

All the results presented in this section have been published in Lara-Rapp and Benavides (2015).

\subsection{Design process characterization}

The Accreditation Board for Engineering and Technology (ABET) defines engineering design as the process of devising a system, component, or process to meet desired needs. According to Pahl et al. (2007), a design process starts off when a need or a set of needs are found in the market. These needs, according to the nomenclature compliant with ISO 9000:2015 and used in the study, can be considered as the requirements. However, costumer's requirements are not the only requirements involved in a project; for example, the organization willing to design the product usually seeks the maximization of profits during the life cycle of the product. This requirement can promote other organization's requirements like the elimination of any action not addressed to preserve or increase the value of the product and so on. Therefore, the objective of the design process is to fulfill all requirements, including the ones of the organization as it was explained in Section 2, where both Designers and Non-Designers Requirements where the objective of the design process (and not only customers'). Based on this concept, this section presents the main aspects for modelling ideal design processes. 


\subsubsection{Useful knowledge}

Useful knowledge, a key aspect for the following results is defined in next Definition 19.

Definition 19: Useful knowledge is the part of the existing knowledge that is applicable during the design process. Inside useful knowledge there are two important pieces of information; Designers and Non-Designers Requirements, which have to be taken into account during the design process.

The ideal situation would be to have all the useful knowledge at the disposal of the design process even though in real processes this may be difficult. Considering that the objective of this section of the thesis is to model an ideal situation in which the design process can reach the best possible results, the availability of all the useful knowledge is assumed hereafter.

The access to all this useful knowledge is not a unique feature of this thesis but it is found in many other studies. For example, Legardeur, Boujut, Tiger (2010) remark that it is important to have access to the whole useful knowledge in the early phases of the design process. Reich (1995) talks about the ideal knowledge, defined as the one which knows all the entities and can describe each of them by abstract concepts without ambiguity. Westerberg et al. (1997) remark how before setting the starting points for the design process it would be necessary to be well aware of what has already been done previously. The concept of all the useful knowledge is also related to the requirements expressed by Ji, Yang and Honda (2012): 1) the need of sufficient information for the design and 2) the need of knowing all the major design alternatives. In the same way, Pahl et al. (2007) explain that due to the central importance of the design process in any organization, it is particularly important for them to make full use of the theoretical knowledge and product experience of designers. It is also important to notice how, in case a big part of the whole useful knowledge is not available, the design process tends to remain faithful to traditional solutions. 
Thus, the result will probably be solutions already proven to be stable and reliable, but the lack of knowledge will avoid exploring new alternatives.

\subsubsection{Ideal Design Process}

According to Matthews (2011), there are two approaches to the design process: the rulebased process and the stochastic process. A stochastic process supports missing or imprecise data and it can provide multiple alternatives for a given input, each with a certain probability. In the rule-based approach, the outcome is deterministic, i.e., for an input, there is a single output. This means that given the useful knowledge to the design process, this process will get a single solution.

This study will employ the rule-based approach for modeling the ideal design processes because it ensures obtaining always the same output by the design process regardless of the design process configuration or the resources consumed. In this way, it permits a fair comparison for proving that a design process which arrives at the best output does not have to be the best one.

The following definitions and properties are introduced for fixing this single output as the one obtained from an ideal rule-based design process.

Definition 20: The Best Output $(B O)$ is the result of a complete interpretation of all the useful knowledge.

Definition 21: The Available Outputs (AvOs) are the result of a partial interpretation of all the useful knowledge.

This definition, aligned with the one provided in Definition 18, indicate that these AvOs are the different alternatives present during a design process which are the candidates for becoming the $\mathrm{BO}$. AvOs do not have to be known a priori but they can be generated as the design process evolves. Thus, the initial number of AvOs, $N$, does not have to be known a priori either. The important fact is that the set of AvOs exists. 
This definition of $N$ has similitudes with the ones found in the literature. For example, it is similar to the one used by Ji, Yang and Honda (2012), where they define it as the total number of possible design alternatives. Westerberg et al. (1997) also use the concept of the space of all the alternatives, similar to the concept of AvOs employed in this study. The concept of AvOs resembles as well the design space of Shah et al. (2003), defined as the count of all possible options for a given problem. For Reich (1995), these $N$ solutions constitute the set of real objects that existed, exists presently or that will exist in the future.

As Westerberg et al. (1997) explain related to their concept of space of alternatives, this space is very large $(N>>1)$. However, in this study this depends on the kind of customer's and organization's requirements. If there are many of them or if they can be hardly satisfied, the number of solutions to be considered can be very limited as Cross $\mathrm{N}$ et al. (1996) explain.

Definition 22: An Ideal Design Process (IDP) is a rule-based design process that obtains the BO.

Note that, by definition, the outcome of a rule-based process is deterministic and thus, Definition 22 leads to a $100 \%$ probability of attaining the BO by the IDP. Consequently, for an IDP, the $\mathrm{BO}$ is always present among the $N$ AvOs, being this $\mathrm{BO}$ unknown a priori by the design process and appearing as a result of it.

As Andreasen and Hein (1987) explain, there are evidences which show that the higher the number of alternative product concepts, the higher the final quality. Shah et al. (2003) and Osborn AF (1963) also suggest how the generation of more ideas increases the chance of better ideas. Hence, in a design process which does not ensure the $\mathrm{BO}$, this can be understood as an evidence supporting that the probability of finding the $\mathrm{BO}$ among a set of $\mathrm{AvOs}$ increases when the number 
of solutions under study increases. However, in the IDP this probability is fixed as per Definition 22.

Property 14: During the time the IDP lasts, the useful knowledge and its interpretation stay constant and hence, the BO stays stationary.

Proof of Property 14: If the useful knowledge or the interpretation is modified at some arbitrary point of the design process, then the BO can be modified. If it is modified, the BO before the change, $\mathrm{BO}_{1}$, is exchanged for $\mathrm{BO}_{2}$, which is a better output than $\mathrm{BO}_{1}$ attending to the interpretation of the new piece of knowledge. Thus, $\mathrm{BO}_{2}$ could have been discarded during the time when $\mathrm{BO}_{1}$ was considered as the $\mathrm{BO}$ and consequently, the design process would not have a $100 \%$ probability of achieving $\mathrm{BO}_{2}$, which is the actual BO. This means that the design process is not an IDP, what contradicts the premise and hence the property must hold.

Definition 23: The Best Design Process (BDP) is an IDP which requires the minimum amount of resources, i.e., it is a process that obtains the $B O$ with the lowest consumption of resources.

Under Definition 23, the BDP has to minimize both the consumption of money and time. If it only minimizes one of the two, it cannot be considered as a BDP. Hence, in the real world, there can be situations where the reduction of the money or time consumed is at the expense of the other. In this case, Definition 23 is not applicable for discriminating which design process is better and this should be decided based on the concrete scenario as well as attending to the priority of each of the resources in that scenario.

Definition 24: The Utopic Design Process (UDP) is the BDP that does not consume any resources.

Although achieving the BO without consuming any resources is a universal organization's requirement, the real world avoids it. This means that the UDP is never attainable, but it is always 
a target. For this reason, the BDP is defined as the IDP with the lowest consumption of resources, which is the nearest IDP to the UDP. Thus, achieving the BDP should be a universal organization requirement.

Following on from the previous definitions, this study proposes that, given the useful knowledge, the resources invested in a design process are related to the acquired complexity (according to the definition of Lu and N. P. Suh [2009]) and hence they can be somehow considered as a measure of it. For this reason, if two design processes arrive at the same solution, the one consuming more resources can be considered as the one introducing a higher acquired complexity during the design process. According to Definition 23, an objective of the design process should be to remove this acquired complexity in order to achieve the BO without any unnecessary consumption of resources.

Note that Definition 20 could be redefined using utility functions and, in this way, the BO would be the one that maximizes a given utility function (for example, Otto and Antonsson [1993] present two of these functions). However, the objective of this study is not to discuss how the BO should be defined (what was done in Section 2), but how the IDP differs from the BDP. Consequently, the only key point for this discussion is that the BO exists and it is well defined as a single solution.

\subsubsection{Phases of the Ideal Design Process}

As it was explained previously, the IDP has access to all the useful knowledge. This knowledge can be interpreted in different ways, leading this to the different phases of the design process. The basic phases of the IDP are, as usual, two: synthesis and analysis. In the first phase the useful knowledge is interpreted for creating (synthesizing) new solutions and ideas whereas in the second phase this useful knowledge is interpreted for assessing (analyzing) the solutions. It is 
important to realize that the useful knowledge is the same for the two of them, the only difference is the way how they interpret this knowledge and, subsequently, use it. Considering that any assessment requires analyzing something previously conceived, the synthesis stage must precede the analysis stage. Hence, the design process can be thought of as a series of synthesis and analysis procedures. In order to describe this ideal synthesis-analysis decomposition, Definitions 25 and 26 are proposed.

Definition 25: An ideal synthesis procedure is a procedure which partially interprets the useful knowledge and generates a set of AvOs.

Attending to Definition 21 and given the useful knowledge, the execution of the ideal synthesis procedure has the required skills for generating the AvOs which, according to Definition 22, always contain the BO. However, the procedure of synthesis cannot identify it due to its partial or incomplete interpretation of the useful knowledge, which is focused on generating AvOs.

Definition 26: An ideal analysis procedure is a procedure which interprets all the useful knowledge and identifies the BO in an absolute way.

An execution of the ideal analysis procedure assesses the suitability of the solution attending to the complete interpretation of the useful knowledge and classifies it as the BO or as not the BO. Definition 26 makes explicit all the useful knowledge to ensure the BO fulfils the maximum number of selection criteria whereas in an absolute way is specified to ensure that, in order to know whether an $\mathrm{AvO}$ is the $\mathrm{BO}$ or not, it does not have to compare it to the others. The reason for the latter stems from the big value that $N$ usually has. When this happens, an evaluation based on absolute characteristics recognizes the BO when it firstly appears and, in this way, it can significantly reduce the consumption of extra resources. The absolute evaluation implies that conditions such as the minimization of the manufacturing costs or the reduction of the 
environmental pollution, have to be substituted for a specific target. This is very important when a design process has to deal with many AvOs (even more when they tend to infinite) since the need of comparing one $\mathrm{AvO}$ with the others can substantially increase the consumption of resources. Thus, the requirement of absolute should be a characteristic of the ideal process. However, this may suppose a limitation in processes where the estimate beforehand of these values is difficult, avoiding in this way the complete definition of the BO.

Property 15: In an IDP, the synthesis procedure has to generate the BO to be identified by the analysis procedure.

Proof of Property 15: If SO's partial interpretation of the useful knowledge generates AvOs which do not satisfy AO's criteria for the BO, then no solution will be selected, what contradicts the definition of an IDP.

If the synthesis and analysis procedure satisfy Property 15, they are called 'compatible'. It is interesting to note that the interpretation of the useful knowledge by the synthesis procedure is different from the one of the analysis procedure due to their different nature: the first is required for proposing solutions and the second is required for assessing the proposed solutions. Although in some models both kinds of interpretations could be merged or confused, this study separates them because 1) the decisions about the output are made in the analytical phase (N. P. Suh 1990) and 2) there are multiple ways for generating alternative product concepts, discussed among others by Zavbi and Rihtarsic (2009).

Property 16: Any IDP based on a replication of synthesis and analysis phases must contain, at least, one ideal procedure of synthesis and one ideal procedure of analysis. 
Proof of Property 16: If an IDP does not have at least one ideal procedure of synthesis and one ideal procedure of analysis, then the BO may not be accessible to the synthesis procedure or the BO may not be identified as such by the analysis procedure.

This property leads to the following definition:

Definition 27: A Replicated Ideal Design Process (IDP-R) is an IDP which replicates at least one procedure of synthesis and one procedure of analysis.

Property 17: Any IDP-R must contain at least two stationary operators used in an iterative way. The first one is the Synthesis Operator (SO): it executes the ideal synthesis procedure creating a set of tentative solutions through an incomplete interpretation of the useful knowledge. The second one is the Analysis Operator (AO): it assesses the proposed solutions using a complete interpretation of all the useful knowledge.

Proof of Property 17: The replication forces the use of the same ideal procedures of synthesis and analysis in those iterations of the IDP where they are used. Definitions 25 and 26 establish that the interpretation of the knowledge of both operators is different: SO is required for generating a set of $A v O s$ and $A O$ for identifying whether a given $A v O$ is the BO or not. Both are needed because, otherwise, the BO could not be generated or identified. As a consequence, an IDP-R can be modeled by means of two different stationary operators (i.e. SO and AO) which are replicated in every iteration.

SO must be previous to $\mathrm{AO}$ since it has to generate AvOs. These AvOs are the input for $\mathrm{AO}$, whose main task is to evaluate and decide whether the $\mathrm{BO}$ is among them or not taking into account the selection criteria of $\mathrm{AO}$. If the $\mathrm{BO}$ is not among them, $\mathrm{AO}$ provides $\mathrm{SO}$ with this new information and the process continues. Now, SO will select another set of AvOs taking into account AO's evaluation of the previous tentative solutions. Thanks to this, SO will not send to 
$\mathrm{AO}$ once again the already-rejected AvOs, what would be pointless. This process conditions the set of AvOs in each step, which will depend on the initial set of AvOs and on the elements already analyzed and rejected in the previous steps. Thus, this set of AvOs is a dynamic set whose elements at any given time constitute a subset of all the previous sets. As already explained, the design process in this model can never change the description of the BO since the useful knowledge of the design process stays stationary.

This separation of the design process in discrete steps constituted by a synthesis operator followed by an analysis operator is similar, but not equal, to the third assumption that Ji, Yang and Honda (2012) use for modeling the design process. As they state, a discussion (i.e., an analysis of the solution) can be divided into time intervals during which designer's preferences are assumed to be unchanged. This assumption establishes that the design process is executed in steps where at each transition between intervals the design team can either keep the most preferred alternative from the previous time interval or change the most preferred alternative to another alternative. However, the design process modeled in the present study differs from the one used by Ji, Yang and Honda (2012) since in that study the BO does not change along the iterative process.

Property 17 states that $\mathrm{SO}$ and $\mathrm{AO}$ are the basic ingredients of an IDP-R sequential model; however, nothing is said about the existence of other operators. In general, there can be other operators which may be added to the sequence of an IDP-R. In this case and in order to model a generic preliminary decision-making tool, the Filter Operator (FO) is introduced. FO represents a preliminary decision-making tool that rejects AvOs which do not satisfy a set of criteria. This generic preliminary analysis can determine whether an $\mathrm{AvO}$ satisfies a given set of characteristics (e.g., the results derived from Theorem 10 in Chapter 2 which, as it was shown there, includes as 
a particular case the axioms from the Axiomatic Design theory by N. P. Suh [1990]) or not, but it cannot identify the BO.

Definition 28: A Filter Operator (FO) is an incomplete interpretation of all the useful knowledge which determines if a subset of AvOs does not contain the BO in absolute terms. Since the filter rejects the elements of such a special subset, the non-rejected AvOs are said to satisfy FO's conditions.

Property 18: In an IDP, the BO, which is fixed by $A O$ 's interpretation, satisfies FO's conditions. Proof of Property 18: If FO's interpretation of the knowledge discards the BO, then no solution will be selected, what contradicts the definition of an IDP.

If FO and AO satisfy Property 18, they are called 'compatible' operators. The concept of FO resembles the one of Reich (1995) in its Theorem 3, where he defines a filter in a design process as a set of design specifications. The description of FO entails that, if a certain solution does not comply with the set of required characteristics imposed by this operator, then the design process discards this solution and gives as a feedback to SO the indication for the removal of that solution from the set of AvOs. However, if the solution satisfies the filter, then this solution is transferred to the analysis operator. It is important to remark that FO does not have the skills of determining if a certain solution is the BO. For this reason Definition 28 says that FO makes a partial interpretation of the useful knowledge, whereas AO makes a complete interpretation of the useful knowledge. It is interesting to note that SO also makes a partial interpretation of the useful knowledge, but the interpretation of FO is more complete than SO's and less complete than AO's, even though in the limit it can be equal to any of them. Finally, the explanation about the absolute evaluation of $\mathrm{FO}$ is the same as the one for $\mathrm{AO}$. 
Property 19: If $M$ is the number of AvOs that satisfy FO's conditions among the $N A v O s$ considered in the design process $(M \leq N)$, then $1 \leq M$ holds.

Proof of Property 19: BO contained in the set of AvOs (see Definition 22) avoids $M=0$ because the maximum subset which does not contain the BO has $N$ - 1 elements.

$M$ is a hidden parameter such as the ones defined by $\mathrm{Ji}$, Yang and Honda (2012). If $M$ is a number close to $N$, then the filter (i.e., the preliminary decision-making tool) does not impose very hard requirements on the ongoing design process. This is usually due to a lack of information beforehand regarding the set of overarching characteristics which will define the BO. However, if $M$ is close to 1, then FO knows in advance most of the main set of key characteristics which will define the BO. Indeed, if $M$ is equal to $N$ it can be said that FO's interpretation of the knowledge is as incomplete as the one of SO. By the same token, if it is equal to 1 , then it can be said that it makes a complete interpretation (equal to AO's). In this way $M$ serves as a measure of the completeness of FO interpretation, being $N$ and 1 their limits.

\subsubsection{The stochastic nature of the Ideal Design Process}

Despite the rule-based approach of the IDP, there is an inbuilt stochastic nature inside the process. Next properties show the source of this stochastic nature.

Property 20: Each $A v O$ can be chosen by $S O$ with a uniform probability distribution.

Proof of Property 20: Definition 25 states that SO has an incomplete interpretation of the useful knowledge valid for generating the AvOs, but not for characterizing the BO or for reducing the number of AvOs. Therefore, all the elements in the set of AvOs have the same probability of being selected by SO.

Property 20 states that all of the $N$ AvOs must have the same probability of being selected, what is equal to the result obtained from the principle of maximum entropy (Jaynes, 1957, Jaynes 
1968). Linked to this, note that the hypothesis of keeping the interpretation of AO away from the interpretation of SO leads to a lack of Shannon's information in SO because AO's interpretation is not contained in $\mathrm{SO}$ and hence, it cannot be used for reducing the entropy in the initial set of AvOs. In this way, although the $\mathrm{BO}$ is deterministic, the output of $\mathrm{SO}$ is a stochastic event due to a lack of skills in this operator for assessing if the chosen solution is the BO. It is worth remarking that, despite some part of the design process has a stochastic approach, the whole process is rulebased and consequently, deterministic. As explained previously, in this process AO will check whether any of the solutions provided by $\mathrm{SO}$ is the $\mathrm{BO}$ and, if not, $\mathrm{SO}$ will continue generating new $\mathrm{AvOs}$ which are evaluated thereupon by $\mathrm{AO}$ until the $\mathrm{BO}$ is found. From this process, it can be inferred that, in spite of the deterministic outcome of the IDP, the number of iterations and the resources consumed for the attainment of the $\mathrm{BO}$ are random variables.

\subsection{Characterization of the resources of the design process}

As Reich and Levy (2004) explain, the design process has to tackle the task of designing a new product under stringent resource constraints with minimal risk. Hence, the organization has to invest wisely the two main resources it has: money and time. These two resources are scarce and the lack of any of them can make the design process unaffordable. For example, the costs involved in a design process usually must match or be under the budget the organization planned for the project. By the same token, the time the organization can invest in the design process is usually bounded and the product must be delivered before a certain date. Next two points will characterize these two resources. 


\subsubsection{Money}

The profits during the life cycle of the product should be estimated before the beginning of the design process or in its early stages, for example, by means of a business plan (see for example Brown and Gutterman [2009] for a detailed explanation about how to develop a business plan). Usually, one of the key parts of a business plan is the projected income statement (Appendix 2 summarizes the main points of an income statement as per the International Accounting Standards Board [2010]). In this study a reduced version of this projected income statement will be used for studying the influence of the design process configuration on the organization's revenues. The idea is to only retain the main points which cover the relations between the design process and the profits coming from the resultant product during its whole life cycle. To this end, Net Income, NIN, is split into two different parts, as presented in Eq. 47: Revenues, RE, and Expenses, E.

$$
N I N=R E-E
$$

The comparison of the design processes will be made assuming that the final product is the same for both design process configurations (this assumption will be discussed at the end of this section), what results in an independency of the revenues with respect to the resources invested during the design process. However, the expenses are inevitably affected by the resources invested during this design process.

In order to isolate the part of the expenses affected by the design process, these are split into two different parts as represented in Eq. 48: the ones related to the design process, $E_{D}$, and the ones independent of the design process, $E_{P}$.

$$
E=E_{D}+E_{P}
$$

$E_{P}$ can include, for example, depreciation and amortization, selling expenses and finance costs. $E_{D}$ represents the expenses of the design process (described, for example, by Kryssanov, 
Tamaki, Kitamura [2001]), including among others the salaries of the professionals working in the design process and all the R\&D activities carried out during the design process. For the sake of the subsequent comparison of design processes, this study will assume that the changes in the design process configuration do not affect the areas of the organization not related to the design process ( $E_{\mathrm{P}}$ is the same). $E_{D}$ can also be divided into fixed, the ones which do not depend on time, and variable expenses, the ones which depend on the time required for reaching the solution.

$$
E_{D}=E_{D F}+E_{D V}
$$

In Eq. $49, E_{D V}$ represents the variable expenses (e.g., salaries or facilities rental), meanwhile $E_{D F}$ represents the expenses independent of how long it takes the design process to reach the solution (e.g., equipment acquisition or patents).

It is important to remark that this description could also have been based on some other measures which could stem, for example, from the cash flow statement. However, in this study the Net Income derived from the income statement is employed to get a measure of the costs of the design process due to its appropriateness to show whether a business has achieved its profitability goal through its operating activities (Needles and Powers 2010).

\subsubsection{Time}

Besides expenses, the other resource of the design process is time. The time available for the design process is limited and will depend on the time required by the different stages involved in the design process. Usually, the shorter it takes the design process to reach the final output, the better. In this study, the time invested in the design process is represented as $T_{D}$.

\subsubsection{Resources properties}

From the previous characterizations, a set of properties is obtained in this section. 
Property 21: If two different IDPs launch one BO each in the market and they do it at the same time, then these IDPs will have the same values of $E_{P}$ and $R$ but they can have a different value of $E_{D}$ and/or $T_{D}$.

Proof of Property 21: By Definition 22, two different IDPs will always arrive at the same output, the BO. Since the two BOs are identical and both are launched in the market at the same time, 1) costumers cannot tell apart one from the other and the price they are willing to pay should be the same ( $R$ is the same), and 2) as explained previously, it is assumed that the changes in the design process configuration do not affect the areas of the organization not related to the design process ( $E_{P}$ is the same). Therefore, two IDPs can only differ in the value of $E_{D}$ and/or $T_{D}$.

It is interesting to note that if the two BOs are not launched at the same time, they will probably get different R. For this reason, Property 21 remarks the premise of the simultaneity in the product launch.

Property 22: The BDP has the lowest value of both $E_{D}$ and $T_{D}$.

Proof of Property 22: By Definition 23, the BDP is the IDP with the lowest consumption of resources. If these resources are time and money, the BDP has to obtain the lowest value of $E_{D}$ and $T_{D}$.

According to Property 22, the objective of the design process is to find the $\mathrm{BO}$ investing the minimum amount of time and money. This permits the use of $E_{D}$ and $T_{D}$ as measures of the distance between a given IDP and the UDP. Furthermore, as Section 4.2.2 explained, this study proposes that for a predetermined set of requirements, $T_{D}$ and $E_{D}$ are related to the acquired complexity (as defined by Lu and N. P. Suh [2009]) and on this account, $E_{D}$ and $T_{D}$ can be somehow considered as measures of it. 


\subsection{Illustrative example}

Inspired by the example provided in Reich (1995) to explain the General Design Theory, the design process of a chair is presented to illustrate the previous concepts. The process starts with the customer's requirement:

Customer's requirement: A comfortable chair easy to transport.

SO, after making a partial interpretation of the useful knowledge it has, decides that the requirement of "comfortable" can be decomposed into the following ones:

- The chair has to be stable for different positions of the person seated on it. Thanks to a set of studies they have access to, the design process sets the requirement of four legs for the chair.

- The upholstery has to be soft, non-allergic and breathable. After considering different materials, they reach the conclusion that the upholstery will be made of cotton since it is the cheapest product which fulfills these conditions based on a set of studies they have access to.

Yet, this list does not define a single chair; there are still many other aspects to be specified. In order to fix these other aspects, SO would need to make a more complete interpretation of the useful knowledge what is out of its reach. Nevertheless, despite SO cannot fix the remaining aspects, it knows (thanks to the partial interpretation of the knowledge) the existence of the following key points for proposing a solution:

\section{Comfort:}

- Is the height of the whole chair adjustable with respect to the floor?

- Are the heights of the armrests and the headrest adjustable?

- Is the inclination of the back adjustable? 


\section{Transportation:}

- Does the chair have wheels?

- Is it made of a light material in order to transport it easily?

- Can it be folded?

All the knowledge related to these aspects (e.g., ergonomic, stability, materials and aesthetics) is part of the useful knowledge needed by the design process to generate the different AvOs. These six aspects can produce many different outputs, which correspond to the different AvOs. In this example, for the sake of simplicity, the number of AvOs is reduced to three $(N=3)$ :

\begin{tabular}{|c|c|c|c|}
\hline Chair & $\mathbf{A}$ & $\mathbf{B}$ & $\mathbf{C}$ \\
\hline \multirow{8}{*}{ Aspects } & \multicolumn{3}{|c|}{ Four legs } \\
\hline & \multicolumn{3}{|c|}{ Cotton upholstery } \\
\hline & \multicolumn{2}{|c|}{ Height of the whole chair adjustable } & Height of the whole chair not adjustable \\
\hline & \multicolumn{2}{|c|}{ Armrest and headrest height adjustable } & $\begin{array}{l}\text { Armrest and headrest height not } \\
\text { adjustable }\end{array}$ \\
\hline & \multicolumn{2}{|c|}{ Back inclination adjustable } & Back inclination not adjustable \\
\hline & \multicolumn{2}{|c|}{ Wheels } & No wheels \\
\hline & Made of aluminium & Made of wood & Made of aluminium \\
\hline & Folding & Not Folding & Folding \\
\hline
\end{tabular}

Table 16: Chair AvOs

AO, making a complete interpretation of the knowledge, sets as the main characteristics of the chair (in order of priority) the following points: 
1) Manufacturing cost: It has to be under $20 €$ per chair to have an acceptable financial margin. The assessment of the cost of the chair entails the preparation of a preliminary budget with a cost of $250 €$ and $8 \mathrm{~h}$.

2) Ergonomics: The chair has to be comfortable and transportable in a set of 50 environments they have identified. The evaluation of every environment means to conduct a survey over 200 persons, who test the conformability and transportation of the chairs during $1 \mathrm{~h}$ in every environment with a cost of $10 € /$ environment / person and $1 \mathrm{~h} /$ environment / person.

Thus, the total cost of an AO analysis can be estimated as $100.250 €$ and 10.008 hours. Next, different design processes are presented:

\section{- Ideal Design Process 1:}

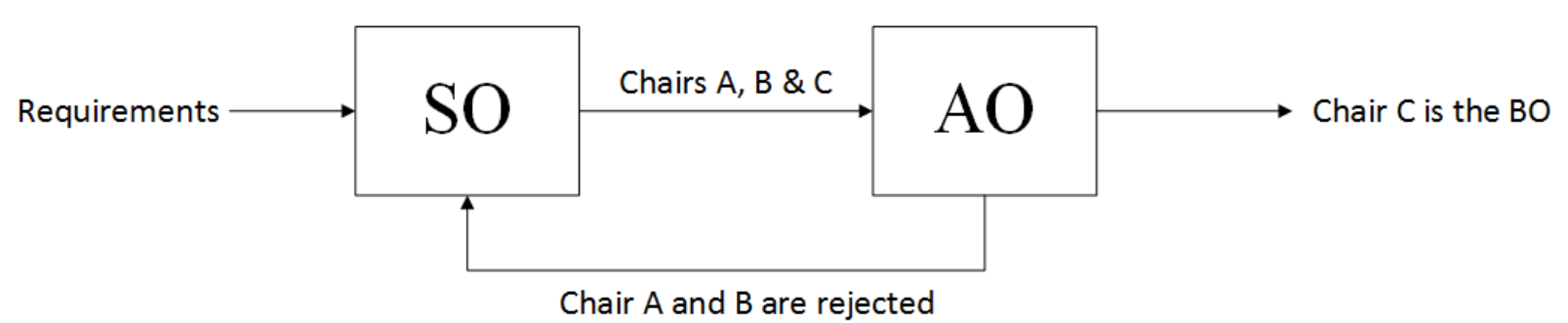

Figure 9: Ideal Design Process 1

Ideal Design Process 1 is composed of one SO and one AO (Figure 9). Chair A is the first one proposed by $\mathrm{SO}$ to $\mathrm{AO}$, which evaluates it and determines that it has a manufacturing cost of $50 €$ per chair and works well in 42 environments, so it is rejected. SO then proposes Chair B to AO, which determines that the manufacturing cost of Chair B is $60 €$ per chair and works well in 50 environments. Consequently, it is discarded. The 
next chair proposed by $\mathrm{SO}$ is Chair $\mathrm{C}$. AO determines that it has a manufacturing cost of $15 €$ and works well in the 50 environments. Hence, Chair C is considered as the BO. In this case, the resources consumed by the design process comprises three times AO's resources, i.e., $300.750 €$ and 30.024 hours, plus the resources consumed by SO for the generation of the AvOs.

\begin{tabular}{|l|c|c|c|}
\cline { 2 - 4 } \multicolumn{1}{c|}{} & $\begin{array}{c}\text { Manufacturing } \\
\text { cost }\end{array}$ & $\begin{array}{c}\text { Successful } \\
\text { environments }\end{array}$ & Result \\
\hline Chair A & $50 €$ & 42 & $\begin{array}{c}\text { Rejected due to manufacturing cost and } \\
\text { ergonomics }\end{array}$ \\
\hline Chair B & $60 €$ & 50 & Rejected due to manufacturing cost \\
\hline Chair C & $15 €$ & 50 & The BO \\
\hline
\end{tabular}

Table 17: Results of Ideal Design Process 1 analysis

\section{- Ideal Design Process 2:}

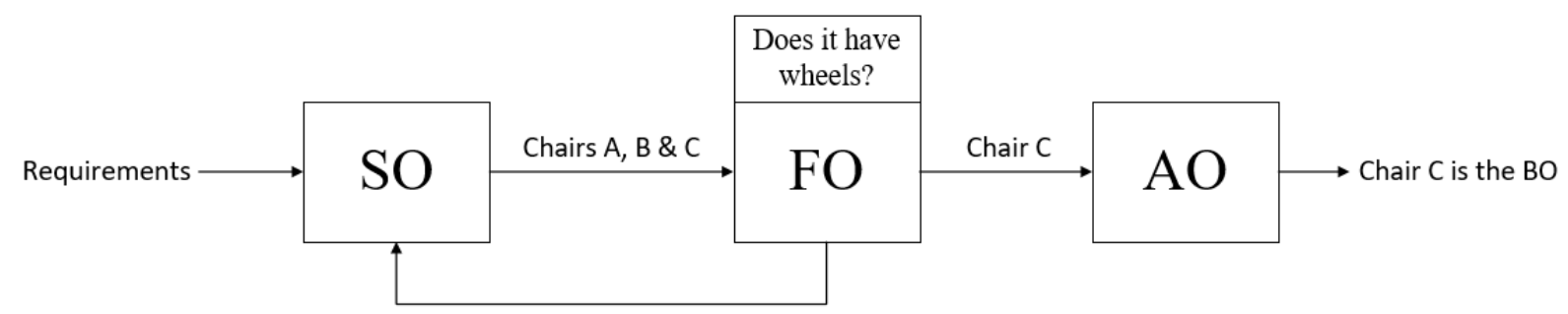

Chair A and B are rejected, no wheels

Figure 10: Ideal Design Process 2 
Ideal Design Process 2 decides to use a preliminary decision-making tool for selecting cheap chairs such that it discards all the chairs with wheels (their interpretation of the useful knowledge indicates that the manufacturing and assembling of the wheels surpass the minimum cost of $20 €$ ). This preliminary decision-making tool will work as FO in this second design process (Figure 10).

$\mathrm{SO}$, as in the previous configuration, proposes firstly Chair A. Then FO checks whether Chair A has wheels or not and, since it has wheels, it is automatically discarded without having to resort to the expensive surveys of AO. The next solution presented by SO is Chair B, again discarded by FO due to the presence of wheels. Eventually, SO proposes Chair C, which satisfies FO's criteria thanks to the lack of wheels. In this case AO executes the evaluations and determines the cost of this chair is $15 €$, leading this to the adoption of Chair $\mathrm{C}$ as the $\mathrm{BO}$.

This preliminary decision-making tool has a value of $M$ equal to 1 in this design process and its cost (just to determine whether the chair has wheels or not) is practically negligible, much more inexpensive and fast than one AO's evaluation. Hence, the total cost of Design Process 2 is lower than the one of 1 since AO only has to evaluate one output (meanwhile Design Process 1 has to analyze the three). For that reason, the total cost is $100.250 €$ and 10.008 hours of AO's evaluation plus the cost of generation of the three AvOs (the same as in Ideal Design Process 1). From this, it is inferred that Ideal Design Process 2 is better than Ideal Design Process 1 due to its lower consumption of resources for reaching the same solution. 


\section{- Design Process 3 (not an IDP):}

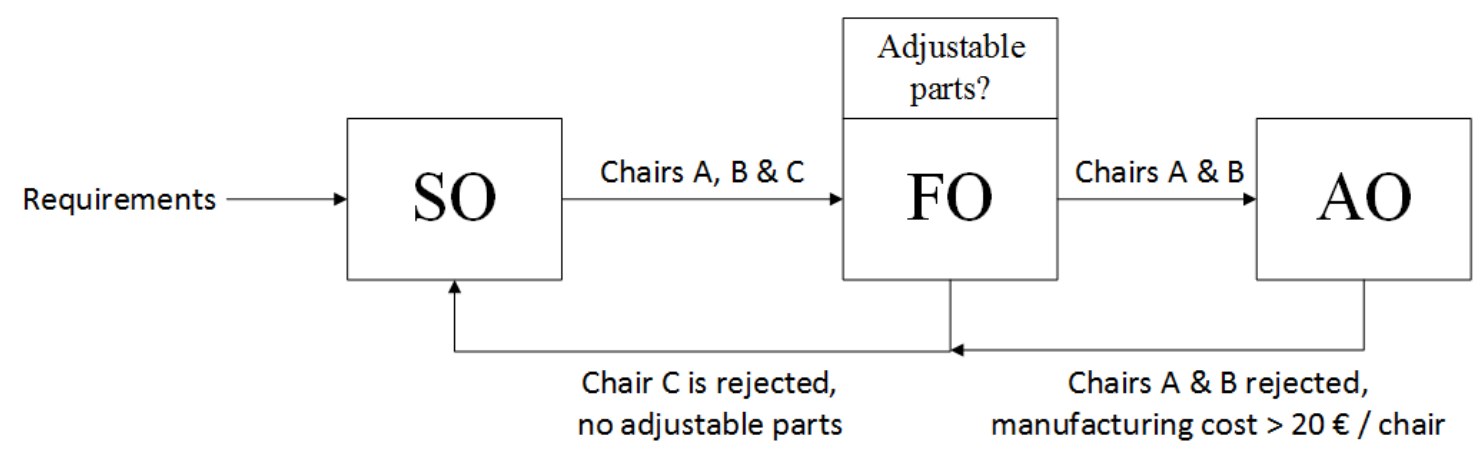

Figure 11: Design Process 3

Design Process 3 decides to use a preliminary decision-making tool for selecting very ergonomic chairs: it discards all the chairs in which the height of the whole chair, the armrests and the headrest are not adjustable (Figure 11). This preliminary decision-making tool will work as FO. However, the problem is that the manufacturing of the mechanisms for adjusting these parts already cost $21 €$ per chair. Hence, no solution will be selected by AO since the manufacturing costs of all the chairs that FO let pass will be over $20 €$. Thus, Chairs A and $\mathrm{B}$ will be rejected by $\mathrm{AO}$ because they have a manufacturing cost of more than $20 €$ per chair and Chair $\mathrm{C}$ will be rejected by FO because it does not have adjustable parts. The result is that the $\mathrm{BO}$ is not achieved and therefore, the design process is not an IDP.

In this case the preliminary decision-making tool (with a value of $M=2$ ) is not compatible with $\mathrm{AO}$, what has led to a catastrophic consequence. Even though the $\mathrm{BO}$ was among the proposed $\mathrm{AvOs}$, it was rejected by FO and no solution was chosen. In this case FO's interpretation or AO's interpretation should be revised. Designers could consider that the interpretation of $\mathrm{AO}$ is not compatible with the customer's requirement because 
comfortable chairs are not usually cheap chairs. However, they could also consider that the interpretation of SO has not created the actual BO, leaving it out of the set of initial AvOs.

\subsection{Evaluation of the impact of preliminary decision- making tools on the consumption of resources and its uncertainty and variability}

In order to evaluate the impact of preliminary decision-making tools on the consumption of resources and the related uncertainty and variability, two different design process configurations are going to be defined based on the design process characterization presented in Sections 4.2 and 4.3; one containing a generic preliminary decision-making tool and another one without it. Then, in Section 4.5.5, both configurations are compared with the objective of assessing under what circumstances a generic preliminary decision-making tool can improve the whole design process.

In the two configurations proposed for this study, the design processes deal with one solution per iteration, what leads to Property 23:

Property 23: Any IDP-R whose SO selects just one AvO per iteration has a maximum number of iterations equal to the number of initial AvOs.

Proof of Property 23: The output of one SO execution is a solution randomly extracted from the set of AvOs according to Property 20. If AO determines that this solution is the BO, the process is finished, if not, the solution is removed from the set of AvOs. Taking into account that this pattern cannot be repeated more times than the number of initial AvOs, the number of SO-AO iterations cannot be larger than the number of initial AvOs. Since, by Property 20, the BO has the same probability as any other solution, if the number of SO-AO iterations is smaller than the number of $A v O s$, there is a probability greater than zero of not selecting the $B O$, what contradicts the fact of having an IDP. Thus, the property holds. 


\subsubsection{Synthesis-Analysis (SA) description}

The first configuration, called SA, is an IDP-R described by the sequence SO-AO-SOAO... (for an example see Section 4.4, IDP 1).

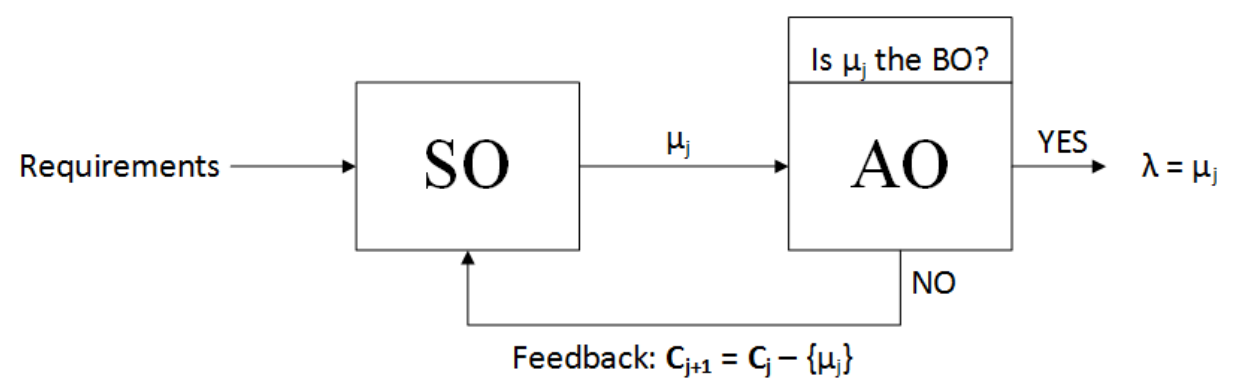

Figure 12: SA flowchart

This design process is modelled as a sequence in which SO starts choosing one random solution among the $N$ AvOs (see Figure 12). Subsequently, AO checks whether this solution is the $\mathrm{BO}$ or not and in case it is, the design process is stopped. However, if the solution chosen by SO is not the $\mathrm{BO}, \mathrm{AO}$ provides $\mathrm{SO}$ with a feedback indicating the removal of this solution from the set of $\mathrm{AvOs}, \mathbf{C}$, before generating another solution. Hence, this set $\mathbf{C}$ will be reduced in the different steps of the process by removing the solutions marked by $\mathrm{AO}$ as not the $\mathrm{BO}$. In order to take into account this step-dependence of the set, $\mathbf{C}_{\mathbf{j}}$ is defined as the set defined by all of the AvOs in the step $j$, being $\mathbf{C}_{\mathbf{0}}$ the initial set such that $\left|\mathbf{C}_{\mathbf{0}}\right|=N$.

Let us call $\mu_{j}$ the solution selected by SO in the step $j$, which is subsequently analyzed by $\mathrm{AO}$ for assessing if $\mu_{j}$ is the $\mathrm{BO}$. In case this solution fulfills all the requirements to be the $\mathrm{BO}$, represented by $\lambda$, then the design process is stopped. However, if $\mu_{j}$ is not the $\mathrm{BO}$, the design process continues providing SO with the previously defined feedback. Table 18 depicts the different results that this process can generate depending on the step $j$ where the $\mathrm{BO}, \lambda$, appears. 


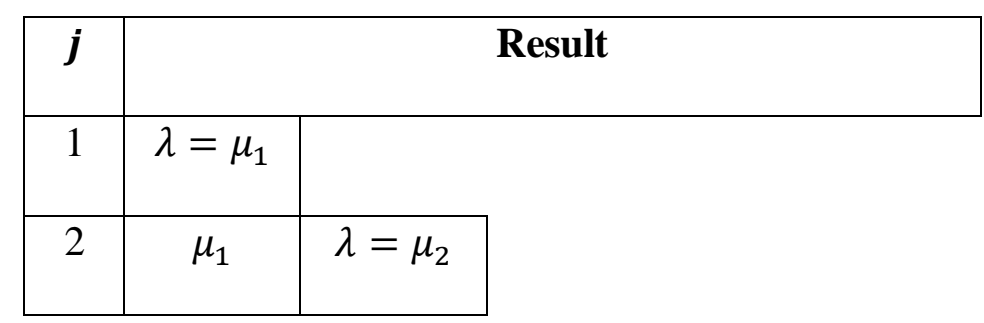

$\ldots$

\begin{tabular}{|l|l|l|l|l|}
\hline $\mathbf{J}$ & $\mu_{1}$ & $\ldots$ & $\mu_{j-1}$ & $\lambda=\mu_{j}$ \\
\hline
\end{tabular}

$\cdots$

\begin{tabular}{|l|l|l|l|l|}
\hline $\mathrm{N}$ & $\mu_{1}$ & $\ldots$ & $\mu_{N-1}$ & $\lambda=\mu_{N}$ \\
\hline
\end{tabular}

Table 18: SA sequence

\subsubsection{Synthesis-Filter-Analysis (SFA) description}

The second configuration, called SFA, is an IDP-R described by the structure SO-FO-[AO] (for an example see Section 4.4, IDP 2). The nomenclature SO-FO-[AO] makes explicit that an iteration could be formed by the sequence SO-FO or by the sequence SO-FO-AO. The difference with respect to SA is that SFA has certain preliminary analysis skills between SO and AO (see Figure 13).

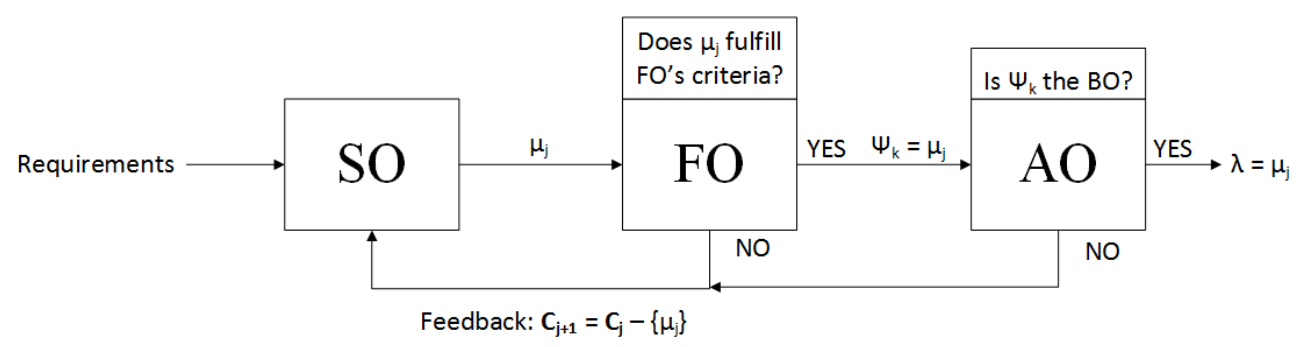

Figure 13: SFA flowchart

As previously, in the first step of this configuration, SO is in charge of choosing randomly one solution among $N$. After the solution has been proposed, FO assesses if this solution fulfills a 
set of predetermined rules which constitute the preliminary decision-making tool. The analysis operator is the same as in the previous configuration; it has the tools to decide whether the solution provided by $\mathrm{SO}$ is the $\mathrm{BO}$ or not. In case it is not the $\mathrm{BO}$, it must provide $\mathrm{SO}$ with a feedback, so that this operator can remove this solution from the set of AvOs. Again, $\mathbf{C}$ will be reduced in the different steps of the process removing the solutions that $\mathrm{AO}$ or $\mathrm{FO}$ has marked as not the $\mathrm{BO}$.

Table 19 depicts the different results that this process can generate depending on the step $j$ where the best solution $\lambda$ appears and the number $k$ representing the number of solutions that have satisfied the filter's conditions. In addition, $\psi_{k}$ represents the $k^{\text {th }}$ solution that has satisfied the filter. As it can be observed, there are $2^{\mathrm{j}-1}$ different ways of obtaining the $\mathrm{BO}$ in the step number $j$. 


\begin{tabular}{|c|c|c|c|c|}
\hline $\boldsymbol{j}$ & $\boldsymbol{k}$ & Case & \multicolumn{2}{|c|}{ Result } \\
\hline 1 & 1 & 1 & $\lambda=\psi_{1}=\mu_{1}$ & \\
\hline
\end{tabular}

\begin{tabular}{|c|c|c|c|c|}
\hline \multirow{2}{*}{2} & 1 & 1 & $\mu_{1}$ & $\lambda=\psi_{1}=\mu_{2}$ \\
\cline { 2 - 5 } & 2 & 2 & $\psi_{1}=\mu_{1}$ & $\lambda=\psi_{2}=\mu_{2}$ \\
\hline
\end{tabular}

\begin{tabular}{|c|c|c|c|c|c|}
\hline \multirow{3}{*}{3} & 1 & 1 & $\mu_{1}$ & $\mu_{2}$ & $\lambda=\psi_{1}=\mu_{3}$ \\
\cline { 2 - 6 } & 2 & 2 & $\mu_{1}$ & $\psi_{1}=\mu_{2}$ & $\lambda=\psi_{2}=\mu_{3}$ \\
\cline { 2 - 6 } & 2 & 3 & $\psi_{1}=\mu_{1}$ & $\mu_{2}$ & $\lambda=\psi_{2}=\mu_{3}$ \\
\cline { 2 - 6 } & 3 & 4 & $\psi_{1}=\mu_{1}$ & $\psi_{2}=\mu_{2}$ & $\lambda=\psi_{3}=\mu_{3}$ \\
\hline
\end{tabular}

$\cdots$

\begin{tabular}{|c|c|c|c|c|c|}
\hline \multirow{3}{*}{$\mathrm{N}$} & 1 & 1 & $\mu_{1}$ & $\cdots$ & $\lambda=\psi_{1}=\mu_{N}$ \\
\cline { 2 - 6 } & $\cdots$ & \multicolumn{5}{|c}{} \\
\cline { 2 - 6 } & $\mathrm{N}$ & $2^{\mathrm{N}-1}$ & $\psi_{1}=\mu_{1}$ & $\cdots$ & $\lambda=\psi_{N}=\mu_{N}$ \\
\hline
\end{tabular}

Table 19: SFA sequence

\subsubsection{Iterations and resources involved}

As Shaja and Sudhakar (2010) explain, when an analysis component proceeds with uncertain inputs in the context of process, process management, multidisciplinary design optimization, product development and so on, the concept of iteration arises. They also pinpoint how important it is to minimize the overall duration and cost associated with this iteration process and hence, to know the number of iterations required for convergence. Next lines will deal with these topics. 
In this study, and based on Property 17, an iteration is defined as the process executed between the selection of one solution and the selection of the next one by SO. This means that in the SA configuration each iteration is the combination of one synthesis process by $\mathrm{SO}$ and one analysis process by AO. In the SFA configuration, an iteration is defined in the following way:

- In case the solution does not satisfy the filter's conditions: one synthesis process by SO and one preliminary analysis process by FO.

- In case the solution satisfies the filter's conditions: one synthesis process by SO, one preliminary analysis process by $\mathrm{FO}$ and an analysis process by $\mathrm{AO}$.

Definition 22 ensures that these two IDPs will always arrive at the same solution. Property 21 ensures that different design processes may differ in the value of $E_{D}$ and/or $T_{D}$, but they have identical values of $E_{P}$ and $R$ when both BOs are launched in the market at the same time. The assumption of launching both BOs into the market at the same time lets this study stay focused on the comparison of the design process per se.

In order to model the resources consumed by these processes (according to Section 4.3), this thesis assumes that every operator has the same variable cost per unit of time (i.e., $E_{D V}^{S}$ for SO, $E_{D V}^{A}$ for $\mathrm{AO}$ and $E_{D V}^{F}$ for $\mathrm{FO}$ are constants) and requires the same amount of time per iteration (i.e., $T_{D}^{S}$ for $\mathrm{SO}, T_{D}^{A}$ for $\mathrm{AO}$ and $T_{D}^{F}$ for $\mathrm{FO}$ are constants). This is a limitation of the model since the time and money consumed by the operators can vary depending on the particular solution. There will be solutions which will require more time and money to be synthesized and analyzed than others. Nevertheless, in principle it is not possible to state for a general problem which iterations are going to demand more resources in the analysis operators ( $\mathrm{AO}$ and $\mathrm{FO}$ ) and this will depend on the particular case to be considered. For this reason, the time and money consumed in every process by the operators are assumed to be an average value of the costs and time required for a 
representative set of processes. These values can be based either on an estimate or on the experience according to Reich and Levy (2004), who explain in their third assumption that the resources linked to the major development tasks to be performed can be estimated with ease by any organization.

\subsubsection{Statistical models}

Note that, based on Property 22, the key point for assessing which design process stays closer to the BDP is to check which one has smaller values of $E_{D}$ and $T_{D}$. However, due to the stochastic nature of the model proposed, $E_{D}$ and $T_{D}$ are also stochastic variables.

This section develops two statistical models in order to evaluate the random variables, one for each of the two configurations. The objective of these models is to reckon the resources spent by each configuration using two statistical functions; expected value and variance. Variance is important because it is a measure of uncertainty and variability, giving an idea of how different the consumption of resources can be from the expected ones and hence, of the risk associated with the design process.

\subsubsection{SA configuration}

In this basic configuration SO generates solutions which AO subsequently studies (see Section 4.5.1). Let us define the random variable $J$, representing the number of solutions analyzed by $\mathrm{AO}$ until $\lambda$ is reached, which takes values from the set $\Phi=\{1,2, . ., N\}$. Attending to Property 20, the possibility of $J=j$ is represented in Eq. 50 (Appendix 3 contains the detailed explanation for this expression). 


$$
P(J=j)=\frac{\left(\begin{array}{c}
N-1 \\
j-1
\end{array}\right)}{\left(\begin{array}{c}
N \\
j-1
\end{array}\right)} \frac{1}{(N-j+1)}=\frac{1}{N}
$$

The mean and the variance of $J$ depend only on the number of AvOs as shown in Eq. 51 and 52. Both of them increase as $N$ grows.

$$
\begin{gathered}
E[J]=\sum_{j=1}^{N} j \cdot P(J=j)=\frac{N+1}{2} \\
\operatorname{Var}[J]=\sum_{j=1}^{N}\left(j-\frac{N+1}{2}\right)^{2} P(J=j)=\frac{N^{2}-1}{12}
\end{gathered}
$$

Since the number of iterations is a random number, $E_{D V}$ in Eq. 49 is also a random variable. In addition, Eq. 49 can be detailed for taking into account the expenses derived from $\mathrm{SO}$ and $\mathrm{AO}$ separately: $E_{D}^{S A}(J)=E_{D F}^{S A}+\left(E_{D V}^{S} T_{D}^{S}+E_{D V}^{A} T_{D}^{A}\right) J$. In this equality $E_{D F}^{S A}$ represents fixed costs whereas $E_{D V}^{S}$ and $E_{D V}^{A}$ are the costs per unit of time of $\mathrm{SO}$ and $\mathrm{AO}$, and $T_{D}^{S}$ and $T_{D}^{A}$ are the times spent by each operator per iteration. Thus, the mean and the variance of the expenses are

$$
\begin{gathered}
E\left[E_{D}^{S A}\right]=\sum_{j=1}^{N} E_{D}^{S A}(J=j) P(J=j)=E_{D F}^{S A}+\left(E_{D V}^{S} T_{D}^{S}+E_{D V}^{A} T_{D}^{A}\right) \frac{N+1}{2} \\
\operatorname{Var}\left[E_{D}^{S A}\right]=\sum_{j=1}^{N}\left(E_{D}^{S A}(J=j)-E\left[E_{D}^{S A}\right]\right)^{2} P(J=j)=\left(E_{D V}^{S} T_{D}^{S}+E_{D V}^{A} T_{D}^{A}\right)^{2} \frac{(N-1)^{2}}{12}
\end{gathered}
$$

Again, both of them increase when the number of AvOs grows. It is worth noting as well that an increment or decrement in the fixed costs does not affect the variance.

The other resource, the time spent in this process, is calculated by means of the following expression: $T_{D}^{S A}(J)=\left(T_{D}^{S}+T_{D}^{A}\right) J$. The mean and the variance of this function depend on the number of AvOs and the time spent by each operator. 


$$
\begin{gathered}
E\left[T_{D}^{S A}\right]=\sum_{j=1}^{N} T_{D}^{S A}(J=j) P(J=j)=\left(T_{D}^{S}+T_{D}^{A}\right) \frac{N+1}{2} \\
\operatorname{Var}\left[T_{D}^{S A}\right]=\sum_{j=1}^{N}\left(T_{D}^{S A}(J=j)-E\left[T_{D}^{S A}\right]\right)^{2} P(J=j)=\left(T_{D}^{S}+T_{D}^{A}\right)^{2} \frac{(N-1)^{2}}{12}
\end{gathered}
$$

These results show that the number of initial AvOs is critical for the expected resources consumed.

Taking the variance as a measure of uncertainty and variability in the design process, a low value of initial AvOs not only reduces the expected consumption of resources, but also reduces the uncertainty and variability (and hence the risk) of the design process. This result is similar to the one obtained by Benavides (2012) using entropic arguments. It is important to remark that this is a consequence of the permanent presence of the BO among the AvOs. In a model where this is not the case, this assertion may not be applicable.

As it can be observed, an increment in the number of initial AvOs can be compensated by a reduction in the time and/or money spent by each operator. Hence, as the number of initial AvOs increases, it is important to be able to reduce the time and the money consumed by each operator. Otherwise, not only the expected consumption of resources grows, but also the evaluation of the consumption of these resources becomes increasingly more uncertain.

\subsubsection{SFA configuration}

In this configuration, $E_{D}$ function is slightly more complex. Now the expenses in each iteration depend on the kind of analysis that the design process has performed up to that moment. For example, if a solution which does not fulfill the filter's requirements is proposed by SO, then the design process only executes one analysis in the iteration: the preliminary analysis in FO (see Section 4.5.2). However, if instead a solution complying with the filter's requirements is selected, 
then the design process carries out two analyses ( $\mathrm{FO} \& \mathrm{AO}$ ), meaning that the design process for this solution should be more expensive in terms of money and time.

The random variable $J$ represents, as in the previous configuration, the number of iterations until $\lambda$ is reached. In addition to $\mathrm{J}$, a new random variable $K$ is introduced, which represents the number of times a solution that fulfills the filter's requirements is selected until $\lambda$ is obtained. $K$ takes values from the set $\Xi=\{1,2, . ., M\}$.

In this case the function representing the expenses of this design process is $E_{D}^{S F A}(J, K)=E_{D F}^{S F A}+\left(E_{D V}^{S} T_{D}^{S}+E_{D V}^{F} T_{D}^{F}\right) J+E_{D V}^{A} T_{D}^{A} K$. The meaning of the different terms is the same as in 4.5.4.1 (now referred to the SFA configuration), with the addition of $E_{D V}^{F}$ representing the cost of FO per unit of time in every iteration and $T_{D}^{F}$ representing the time invested by FO in every iteration. Again, $J$ takes values from the set $\Phi=\{1,2, . ., N\}$. The possibility of finishing the design process in $J=j$ steps is presented in Eq. 57.

$$
P(J=j)=\frac{\left(\begin{array}{c}
N-1 \\
j-1
\end{array}\right)}{\left(\begin{array}{c}
N \\
j-1
\end{array}\right)} \frac{1}{(N-j+1)}=\frac{1}{N}
$$

This result is equal to the one of SA configuration, a uniform distribution (see Appendix 3). Hence, the expected value and variance are the same as the ones obtained in the previous configuration.

Regarding the probability distribution of K, Eq. 58 shows the probability of getting $k \psi$ solutions in $j$ draws from a finite population of size $N$ which contains $M \psi$-solutions. 


$$
P(K=k \mid J=j)=\frac{\left(\begin{array}{c}
M-1 \\
k-1
\end{array}\right)\left(\begin{array}{c}
N-M \\
j-k
\end{array}\right)}{\left(\begin{array}{c}
N-1 \\
j-1
\end{array}\right)}
$$

This is the definition of a hypergeometric distribution, whose properties can be consulted in Hayter (2012). The expected value of getting $k \psi$-solutions and its variance are calculated in Eqs. 59 and 60 respectively, which only depend on the value of initial AvOs satisfying the filter. As this value grows, both of them increase. It is also important to remark that neither the expected value nor the variance depends on the initial number of AvOs.

$$
\begin{gathered}
E[K]=\sum_{j=1}^{N} \sum_{k=1}^{M} P(K=k \mid J=j) P(J=j) k=\frac{M+1}{2} \\
\operatorname{Var}[K]=\sum_{j=1}^{N} \sum_{k=1}^{M} P(K=k \mid J=j) P(J=j)\left(k-\frac{M+1}{2}\right)^{2}=\frac{M^{2}-1}{12}
\end{gathered}
$$

The expected value and the variance of the expenses function, $E_{D}^{S F A}$, are calculated in next Eqs. 61 and 62 respectively. These values show a dependence with the number of initial AvOs as well as with the number of solutions satisfying the filter and the money spent by each operator, being this behavior very similar to the one explained in Section 4.5.4.1.

$$
\begin{gathered}
E\left[E_{D}^{S F A}\right]=\sum_{k=1}^{M} \sum_{j=1}^{N} E_{D}^{S F A}(J=j, K=K) P(K=k \mid J=j) P(J=j) \\
=E_{D F}^{S F A}+\left(E_{D V}^{S} T_{D}^{S}+E_{D V}^{F} T_{D}^{F}\right) \frac{N+1}{2}+E_{D V}^{A} T_{D}^{A} \frac{M+1}{2} \\
\operatorname{Var}\left[E_{D}^{S F A}\right]=\sum_{j=1}^{N} \sum_{k 1}^{M}\left(E_{D}^{S F A}(J=j, K=k)-E\left[E_{D}^{S F A}\right]\right)^{2} P(K=k \mid J=j) P(J=j) \\
=\frac{\left(E_{D V}^{A} T_{D}^{A}\right)^{2}}{12}\left(M^{2}-1\right)+\frac{\left(E_{D V}^{S} T_{D}^{S}+E_{D V}^{F} T_{D}^{F}\right)^{2}}{12}\left(N^{2}-1\right)+\frac{\left(E_{D V}^{S} T_{D}^{S}+E_{D V}^{F} T_{D}^{F}\right) E_{D V}^{A} T_{D}^{A}}{6}(N+1)(M-1)
\end{gathered}
$$


The amount of time invested in this configuration is calculated by means of $T_{D}^{S F A}(J, K)=\left(T_{D}^{S}+T_{D}^{F}\right) J+T_{D}^{A} K$. The expected value of $T_{D}^{S F A}$ and its variance are calculated in Eqs. 63 and 64, depending both of them on $N, M$ and the time spent by each operator.

$$
\begin{gathered}
E\left[T_{D}^{S F A}\right]=\sum_{j=1}^{N} \sum_{k=1}^{M} T_{D}^{S F A}(J=j, K=k) P(K=k \mid J=j) P(J=j) \\
=\left(T_{D}^{S}+T_{D}^{F}\right) \frac{N+1}{2}+T_{D}^{A} \frac{M+1}{2} \\
\operatorname{Var}\left[T_{D}^{S F A}\right]=\sum_{j=1}^{N} \sum_{k=1}^{M}\left(T_{D}^{S F A}(J=j, K=k)-E\left[T_{D}^{S F A}\right]\right)^{2} P(K=k \mid J=j) P(J=j) \\
=\frac{\left(T_{D}^{A}\right)^{2}}{12}\left(M^{2}-1\right)+\frac{\left(T_{D}^{S}+T_{D}^{F}\right)^{2}}{12}\left(N^{2}-1\right)+\frac{\left(T_{D}^{S}+T_{D}^{F}\right) T_{D}^{A}}{6}(N+1)(M-1)
\end{gathered}
$$

\subsubsection{Relation between design process and design department}

The structure of Eqs. 61 to 64 shows how the terms related to SO and FO appear together, suggesting the possibility of melding SO and FO into one single operator which can be called Advanced Synthesis Operator (ASO), similar to the Advanced Design Theories introduced by Benavides (2012). This operator, besides the synthesis ability, would have some preliminary analysis capacities provided by the preliminary decision-making tool which is able to filter the results generated in the synthesis stage that subsequently arrive at AO. This could lead to a design department with two groups; one for the advanced synthesis stage and another one for the analysis stage.

\subsubsection{SA \& SFA comparison}

The next four points compare the difference in the consumption of resources between the two design process configurations. The objective is to assess under what conditions the 
introduction of FO improves the design process in terms of time and expenses. This improvement is measured by the relative improvement function:

$$
\eta_{z}(x)=1-\frac{Z_{S F A}(x)}{Z_{S A}(x)}
$$

where $Z$ identifies the parameter to be compared and $x$ the ratio between the resources consumed by $\mathrm{FO}$ and $\mathrm{AO}$ in each iteration.

\subsubsection{1. $\quad$ Expected time}

The relative improvement between the expected time it takes SA to arrive at the $\mathrm{BO}$ and the expected time it takes SFA to do the same is defined in the following way:

$$
\eta_{E[T]}(t)=1-\frac{E\left[T_{D}^{S F A}\right](t)}{E\left[T_{D}^{S A}\right]}=\beta_{E[T]}\left(\alpha_{E[T]}-t\right)
$$

where Eqs. 55 and 63 have been used for defining the following coefficients:

$$
\begin{gathered}
\alpha_{E[T]}=\frac{N-M}{N+1} \\
\beta_{E[T]}=\frac{T_{D}^{A}}{T_{D}^{S}+T_{D}^{A}} \\
t=\frac{T_{D}^{F}}{T_{D}^{A}}
\end{gathered}
$$

Hence, the relative improvement depends on $t$, the number of initial AvOs and the number of them which satisfy the filter's conditions. Parameters $t, \alpha_{E[T]}$ and $\beta_{\mathrm{E}[\mathrm{T}]}$ are always positive and hence, $d \eta_{\mathrm{E}[\mathrm{T}]}(t) / d t=-\beta_{\mathrm{E}[\mathrm{T}]}<0$. This means that when $t$ increases, $\eta_{\mathrm{E}[\mathrm{T}]}(t)$ decreases, reducing in this way the temporal profits of having a filter in the configuration. Figure 14 shows this relation; the lower $t$ is, the higher the advantage of using a preliminary decision-making tool is. 


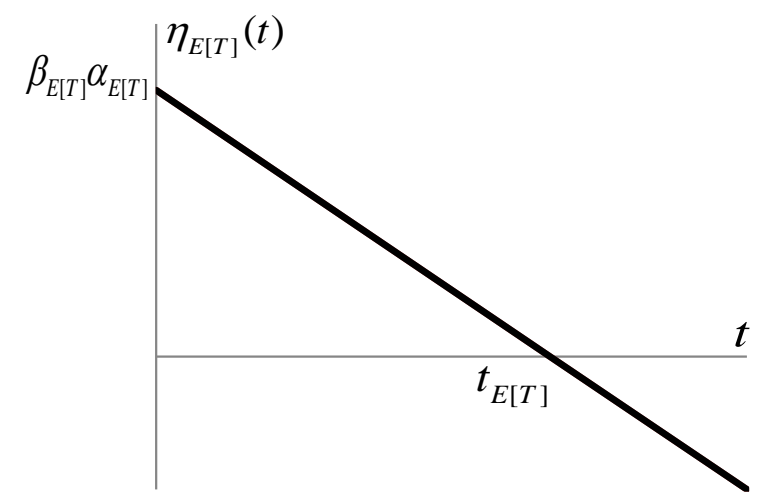

Figure 14: Difference between SA and SFA expected time

The value of $t$ which equals the expected time for both configurations is $t_{E[T]}=\alpha_{\mathrm{E}[\mathrm{T}]}>0$.

If $t$ is higher than $t_{E[T]}$, then the expected time it takes SFA to arrive at the BO is higher than the expected time it takes SA. It is also interesting to note that the bigger $t_{E[T]}$ is, the better, since it means there is a wider interval of $t$ where the preliminary design tool improves the expected time. Hence, a good filter should have a small number of solutions satisfying it compared to the initial number of AvOs. However, if all the proposed solutions satisfy the filter (i.e., $N=M$ ), then the filter will not improve the expected time invested in the design process, no matter how small $t$ is.

The maximum improvement is obtained when the preliminary decision-making tool is such that $N \gg M$, what maximizes the value of $\alpha_{E[T]}$ and hence, also maximizes the maximum value of the improvement function (achievable when $t \rightarrow 0$ ) together with the value of $t_{E[T]}$. At the same time, when $N \gg M$, the tendency $t_{E[T]} \rightarrow 1$ holds, but it always remain smaller than 1 since the minimum value of $M$ is one (and hence $t_{E[T]}=1$ can never be reached). 


\subsubsection{Variance of time}

This section evaluates under what conditions the variance of the time it takes SFA to arrive at the solution is lower than SA's one. The relative improvement is:

$$
\eta_{\operatorname{Var}[T]}(t)=1-\frac{\operatorname{Var}\left[T_{D}^{S F A}\right](t)}{\operatorname{Var}\left[T_{D}^{S A}\right]}=\alpha_{\operatorname{Var}[T]}-t \beta_{\operatorname{Var}[T]}-t^{2} \gamma_{\operatorname{Var}[T]}
$$

The parameters in Eq. 70 depend only on the number of initial AvOs, the number of them satisfying the filter's condition and the relation between the time spent in every iteration by SO and AO:

$$
\begin{gathered}
\alpha_{\operatorname{Var}[T]}=1-\gamma_{\operatorname{Var}[T]}\left[\left(\frac{T_{D}^{S}}{T_{D}^{A}}\right)^{2}+\frac{M^{2}-1}{N^{2}-1}+2 \frac{T_{D}^{S}}{T_{D}^{A}} \frac{M-1}{N-1}\right] \\
\beta_{\operatorname{Var}[T]}=2 \gamma_{\operatorname{Var}[T]}\left[\frac{M-1}{N-1}+\frac{T_{D}^{S}}{T_{D}^{A}}\right]>0 \\
\gamma_{\operatorname{Var}[T]}=\frac{\left(T_{D}^{A}\right)^{2}}{\left(T_{D}^{S}+T_{D}^{A}\right)^{2}} \frac{N+1}{N-1}>0
\end{gathered}
$$

The first and the second derivative are

$$
\begin{gathered}
\frac{d \eta_{\operatorname{Var}[T]}(t)}{d t}=-\beta_{\operatorname{Var}[T]}-2 t \gamma_{\operatorname{Var}[T]} \\
\frac{d^{2} \eta_{\operatorname{Var}[T]}(t)}{d t^{2}}=-2 \gamma_{\operatorname{Var}[T]}<0
\end{gathered}
$$

The first derivative is zero for $t_{M}=-\beta_{\operatorname{Var}[T]} / 2 \gamma_{\operatorname{Var}[T]}<0$. Taking into account that Eq. 75 indicates that the function is concave, then $t_{M}$ is a maximum. This maximum is obtained for a value of $t$ lower than zero, which is out of the boundaries. In addition, depending on the value of $\alpha_{\operatorname{Var}[T]}$ , there are two different situations represented in Figure 15. 


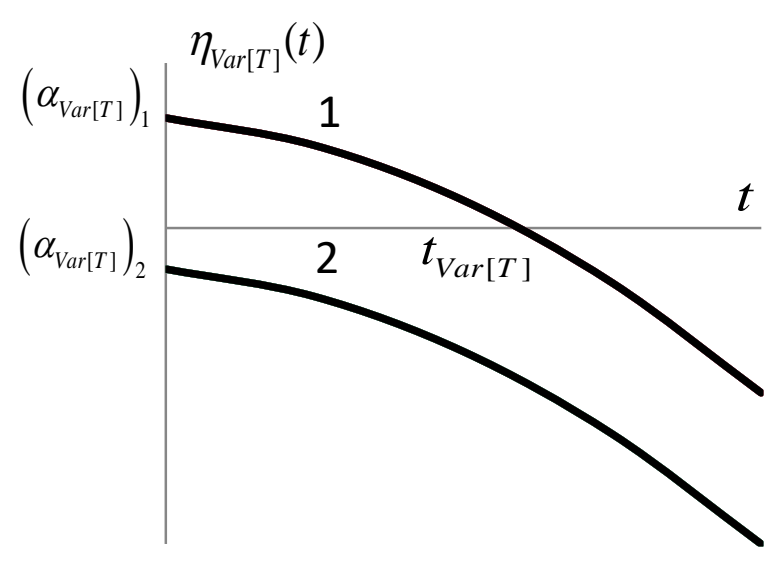

Figure 15: Difference between SA and SFA in terms of variance of time. Cases $1 \& 2$

When $\alpha_{\operatorname{Var}[T]}<0$, SA configuration has a lower variance in the time it takes it to arrive at the BO than SFA for all values of $t$ (line 2). When $\alpha_{\operatorname{Var}[T]}>0$ (line 1), the temporal variance of SFA is lower than SA's up to $t_{\operatorname{Var}[T]}$, where they are equal. If $t$ starts growing from this value on, the variance of the time of SFA starts increasingly worsening with respect to the one of SA. This value of $t_{\operatorname{Var}[T]}$ is represented in Eq. 76 .

$$
\eta_{\operatorname{Var}[T]}(t)=0 \rightarrow\left(t_{\operatorname{Var}[T]}\right)_{1,2}=-\frac{\beta_{\operatorname{Var}[T]} \pm \sqrt{\beta_{\operatorname{Var}[T]}^{2}+4 \alpha_{\operatorname{Var}[T]} \gamma_{\operatorname{Var}[T]}}}{2 \gamma_{\operatorname{Var}[T]}}
$$

However, from the two solutions of Eq. 76, the negative value of the time has no sense. Thus, the positive result is presented in Eq. 77.

$$
t_{\operatorname{Var}[T]}=\frac{1}{2}\left(-\frac{\beta_{\operatorname{Var}[T]}}{\gamma_{\operatorname{Var}[T]}}+\sqrt{\left(\frac{\beta_{\operatorname{Var}[T]}}{\gamma_{\operatorname{Var}[T]}}\right)^{2}+4 \frac{\alpha_{\operatorname{Var}[T]}}{\gamma_{\operatorname{Var}[T]}}}\right)
$$


A reduction in the variance requires a positive value for $\alpha_{\operatorname{Var}[T]}$ :

$$
\alpha_{\operatorname{Var}[T]}>0 \rightarrow 1>\gamma_{\operatorname{Var}[T]}\left[\left(\frac{T_{D}^{S}}{T_{D}^{A}}\right)^{2}+\frac{M^{2}-1}{N^{2}-1}+2 \frac{T_{D}^{S}}{T_{D}^{A}} \frac{M-1}{N-1}\right]
$$

It is also remarkable that the bigger $t_{\operatorname{Var}[T]}$, the wider the interval of $t$ where the preliminary design tool improves the variance of the invested time and hence, the better the situation. This result agrees with the one obtained for $t_{E[T]}$ in Section 4.5.5.1.

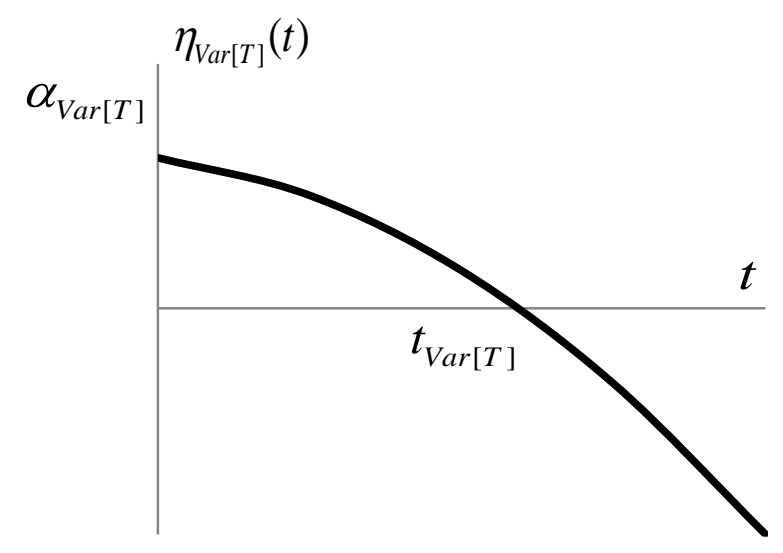

Figure 16: Difference between SA and SFA variance of time in case 1

Figure 16 shows that, when $\alpha_{\operatorname{Var}[T]}>0$, the lower $t$, the higher the reduction of the variance of the time invested in the design process. Putting together this result with the one obtained in 4.5.5.1, they conclude that a preliminary design tool with a small value of $t$ reduces both the expected time it takes the design process to arrive at the $\mathrm{BO}$ and the variance of this time.

An analysis process can involve simulations, tests, prototypes, etc., whereas a synthesis process can be executed by means of fast methods like brain-storming (see Osborn [1963] for further information and examples) or the morphological matrix (Weber and Sridhar 1998). Therefore, this leads to think that a synthesis process normally consumes far less time than the 
analysis of the solution, $\left(T_{D}^{S} / T_{D}^{A} \ll<1\right)$. In this case, Eq. 78 would be simplified into $(N-1)^{2}>M^{2}-1$. In a design process with a preliminary-decision tool such that $N>M$ this inequality is always satisfied and the decision-making tool, depending on the value of $t$, can greatly improve the variance of the time.

The maximum improvement is attained when the preliminary decision-making tool is such that $N \gg M$ and $T_{D}^{S} / T_{D}^{A} \ll<1$, which maximizes the value of $\alpha_{\operatorname{Var}[T]}$ and hence, also maximizes the maximum value of the improvement function. This last point is achieved when $t \rightarrow 0$. In this situation the tendency $t_{\operatorname{Var}[T]} \rightarrow 1$ holds.

\subsubsection{3. $\quad$ Expected expenses}

This section compares the expected expenses generated by SA and SFA depending on the value of $e=E_{D V}^{F} T_{D}^{F} / E_{D V}^{A} T_{D}^{A}=t E_{D V}^{F} / E_{D V}^{A}$. This ratio, which has to be positive to make sense, represents the relation between the expenses generated in each iteration by the preliminary analysis operator (FO) and the expenses generated by the detailed analysis operator (AO). The corresponding relative improvement is

$$
\eta_{\mathrm{E}[\mathrm{E}]}(e)=1-\frac{E\left[E_{D}^{S F A}\right](e)}{E\left[E_{D}^{S A}\right]}=\beta_{\mathrm{E}[\mathrm{E}]}\left(\alpha_{\mathrm{E}[\mathrm{E}]}-e\right)
$$

where:

$$
\begin{gathered}
\alpha_{\mathrm{E}[\mathrm{E}]}=\frac{1}{N+1}\left[2 \frac{E_{D F}^{S A}-E_{D F}^{S F A}}{E_{D V}^{A} T_{D}^{A}}+N-M\right] \\
\beta_{\mathrm{E}[\mathrm{E}]}=\frac{E_{D V}^{A} T_{D}^{A}(N+1)}{2 E_{D F}^{S A}+\left(E_{D V}^{S} T_{D}^{S}+E_{D V}^{A} T_{D}^{A}\right)(N+1)}>0
\end{gathered}
$$


Eq. 79 indicates a linear relation between $\eta_{E[E]}$ and $e$. Since $d \eta_{E[E]}(e) / d e=-\beta_{\mathrm{E}[\mathrm{E}]}<0$, $\eta_{E[E]}$ decreases when $e$ grows. Depending on the value of $\alpha_{E[E]}$, one of the two situations represented in Figure 17 takes place.

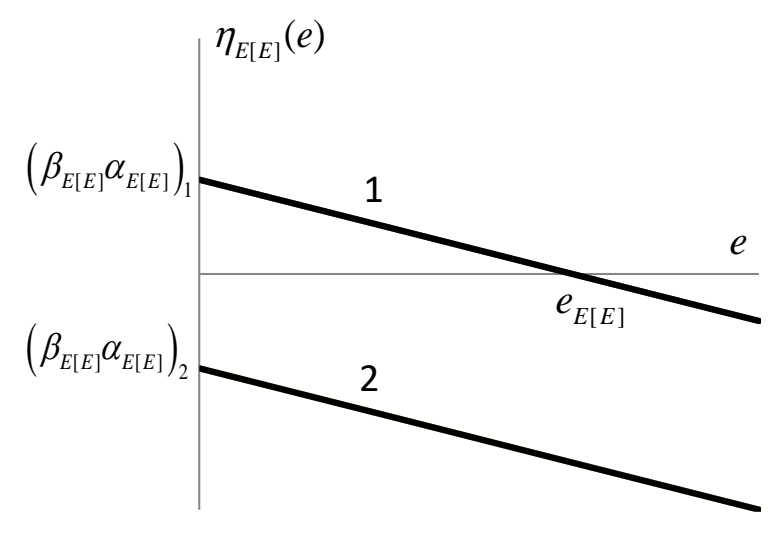

Figure 17: Difference between SA and SFA expected expenses. Cases $1 \& 2$

When $\alpha_{\mathrm{E}[\mathrm{E}]}$ is positive, there are some values of $e$ for which SFA configuration provides lower expected costs than SA configuration (line 1 in Figure 17). The condition is

$$
\frac{N-M}{2}>\frac{E_{D F}^{S F A}-E_{D F}^{S A}}{E_{D V}^{A} T_{D}^{A}}
$$

However, when $\alpha_{\mathrm{E}[\mathrm{E}]}$ is negative (line 2 in Figure 17), SFA always results in higher expected costs than SA. The ratio at which $\eta_{\mathrm{E}[\mathrm{E}]}=0$ (only meaningful when $\alpha_{\mathrm{E}[\mathrm{E}]}$ is positive, line 1) represents the situation when the expected expenses of the two configurations are the same: $e_{E[E]}=\alpha_{\mathrm{E}[\mathrm{E}]}>0$. When $\alpha_{\mathrm{E}[\mathrm{E}]}$ is higher than zero, there are two different situations (see Figure 18): 1) if $e$ is higher than $e_{\mathrm{E}[\mathrm{E}]}$, then the expected expenses generated by $\mathrm{SA}$ are lower than the ones by SFA and 2) if this value is smaller than $e_{\mathrm{E}[\mathrm{E}]}$ the opposite situation results. 


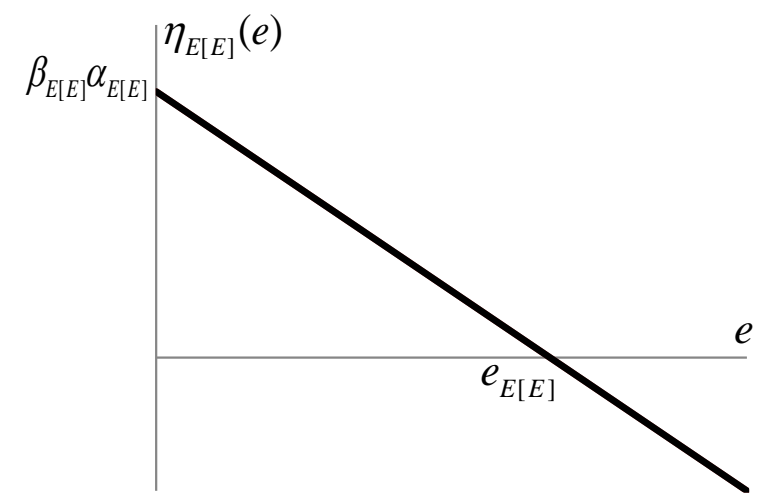

Figure 18: Difference between SA and SFA expected expenses in case 1

Figure 18 shows that in case 1 , the lower the value of $e$ is, the higher the improvement of the preliminary decision-making tool with respect to the expected expenses is. As mentioned previously with the other parameters, the best situation is to have this interval as wide as possible and consequently, to have the fixed expenses of SFA configuration, $E_{D F}^{S F A}$, as low as possible. The maximum improvement is obtained when the preliminary decision-making tool is such that $N>>$ $M$, which maximizes the value of $\alpha_{E[E]}$ and thus, also maximizes the maximum value of the improvement function (achievable when $e \rightarrow 0$ ) together with the value of $t_{E[E]}$. In this situation $(N>>M), e_{\mathrm{E}[\mathrm{E}]}$ tends to $1+2\left(E_{D F}^{S A}-E_{D F}^{S F A}\right) /\left(N E_{D V}^{A} T_{D}^{A}\right)$.

\subsubsection{Variance of expenses}

This section evaluates the conditions which lead to a lower variance of the expenses in SFA than in SA depending on $e$. The relative improvement of the variance of the expenses is

$$
\eta_{\operatorname{Var}[E]}(e)=1-\frac{\operatorname{Var}\left[E_{D}^{S F A}\right](e)}{\operatorname{Var}\left[E_{D}^{S A}\right]}=\alpha_{\operatorname{Var}[E]}-e \beta_{\operatorname{Var}[E]}-e^{2} \gamma_{\operatorname{Var}[E]}
$$

where 


$$
\begin{gathered}
\alpha_{\operatorname{Var}[E]}=1-\gamma_{\operatorname{Var}[E]}\left(\left(\frac{E_{D V}^{S} T_{D}^{S}}{E_{D V}^{A} T_{D}^{A}}\right)^{2}+\frac{M^{2}-1}{N^{2}-1}+2 \frac{E_{D V}^{S} T_{D}^{S}}{E_{D V}^{A} T_{D}^{A}} \frac{M-1}{N-1}\right) \\
\beta_{\operatorname{Var}[E]}=2 \gamma_{\operatorname{Var}[E]}\left[\frac{M-1}{N-1}+\frac{E_{D V}^{S} T_{D}^{S}}{E_{D V}^{A} T_{D}^{A}}\right]>0 \\
\gamma_{\operatorname{Var}[E]}=\frac{\left(E_{D V}^{A} T_{D}^{A}\right)^{2}}{\left(E_{D V}^{S} T_{D}^{S}+E_{D V}^{A} T_{D}^{A}\right)^{2}} \frac{N+1}{N-1}>0
\end{gathered}
$$

The first and second derivative are

$$
\begin{gathered}
\frac{d \eta_{\operatorname{Var}[E]}(e)}{d e}=-\beta_{\operatorname{Var}[E]}-2 e \gamma_{\operatorname{Var}[E]} \\
\frac{d \eta_{\operatorname{Var}[E]}(e)}{d e}=0 \rightarrow e_{M}=-\frac{\beta_{\operatorname{Var}[E]}}{2 \gamma_{\operatorname{Var}[E]}}<0 \\
\frac{d^{2} \eta_{\operatorname{Var}[E]}(e)}{d e^{2}}=-2 \gamma_{\operatorname{Var}[E]}<0
\end{gathered}
$$

Eq. 89 shows the function is concave and consequently, $e_{M}$ is a maximum. This maximum is obtained for a value of $e$ lower than zero, which is out of the meaningful region. Depending on the value of $\alpha_{\operatorname{Var}[E]}$, there are two different situations (represented in Figure 19).

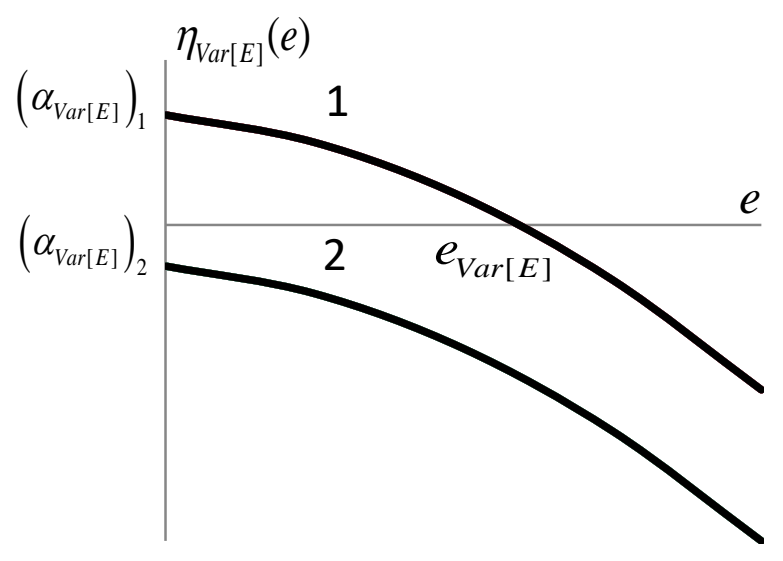

Figure 19: Difference between SA and SFA variance of expenses. Cases $1 \& 2$ 
When $e_{\operatorname{Var}[E]}<0$, SA configuration has lower expenses than SFA for any value of $e$. If $e_{\operatorname{Var}[E]}>0$ (line 1 in Figure 19), then SFA expenses show a lower variance than SA up to $e_{\operatorname{Var}[E]}$ where they are equal. If $e$ continues increasing, then the variance of the expenses of SA becomes lower than the one in SFA. The value of $e_{\operatorname{Var}[E]}$ is such that

$$
\eta_{\operatorname{Var}[E]}(e)=0 \rightarrow\left(e_{\operatorname{Var}[E]}\right)_{1,2}=-\frac{\beta_{\operatorname{Var}[E]} \pm \sqrt{\beta_{\operatorname{Var}[E]}^{2}+4 \alpha_{\operatorname{Var}[E]} \gamma_{\operatorname{Var}[E]}}}{2 \gamma_{\operatorname{Var}[E]}}
$$

Since $e$ must be positive, the negative solution is neglected.

$$
e_{\operatorname{Var}[E]}=\frac{1}{2}\left(-\frac{\beta_{\operatorname{Var}[E]}}{\gamma_{\operatorname{Var}[E]}}+\sqrt{\left(\frac{\beta_{\operatorname{Var}[E]}}{\gamma_{\operatorname{Var}[E]}}\right)^{2}+4 \frac{\alpha_{\operatorname{Var}[E]}}{\gamma_{\operatorname{Var}[E]}}}\right)
$$

For the same reason as the one explained in Section 4.5.5.1, but now applied to the variance of expenses, a high value of $e_{\operatorname{Var}[E]}$ is interesting.

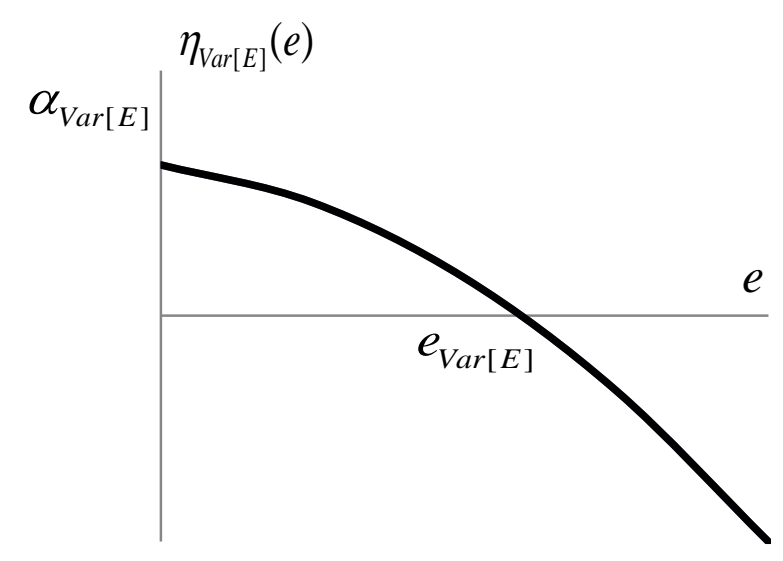

Figure 20: Difference between SA and SFA variance of time in the case 1 
Figure 20 shows how the lower the value of $e$, the higher the improvement on the variance of the expenses during the design process. This result is similar as the one obtained in Section 4.5.5.3, where a smaller value of $e$ led to a higher improvement on the expected expenses. Putting together the two results, it can be inferred that the smaller the value of $e$, the higher the improvement in the expected expenses and its variance. This result shows that a situation where $\alpha_{\operatorname{Var}[E]}>0$ is desirable, what implies

$$
1>\gamma_{\operatorname{Var}[E]}\left(\left(\frac{E_{D V}^{S} T_{D}^{S}}{E_{D V}^{A} T_{D}^{A}}\right)^{2}+\frac{M^{2}-1}{N^{2}-1}+2 \frac{E_{D V}^{S} T_{D}^{S}}{E_{D V}^{A} T_{D}^{A}} \frac{M-1}{N-1}\right)
$$

This inequality can be simplified assuming that the synthesis of a solution requires much less money than the analysis of the chosen solution in every step, i.e., $E_{D V}^{S} T_{D}^{S} \ll<E_{D V}^{A} T_{D}^{A}$. This is not a strange situation since, as it has been explained in Section 4.5.5.2, a synthesis process usually can be carried out by means of simpler tools than an analysis process, which may entail an investment in prototypes, tests, etc. In this case Eq. 92 is simplified into: $(N-1)^{2}>\left(M^{2}-1\right)$. In a design process such that $N>M$, the previous relation is satisfied and the decision-making tool improves the expected time depending on the value of $e$.

The maximum improvement is attained when the preliminary decision-making tool is such that $N \gg M$ and $E_{D V}^{S} T_{D}^{S} \ll<E_{D V}^{A} T_{D}^{A}$, which maximizes the value of $\alpha_{\operatorname{Var}[E]}$ and hence, also maximizes the maximum value of the improvement function, which is achieved when $e \rightarrow 0$. In this situation the tendency $e_{\operatorname{Var}[E]} \rightarrow 1$ holds.

\subsubsection{Summary}

Next Table 20 contains, based on the results of the comparison performed in Section 4.5.5, the different conditions the preliminary decision-making tool has to fulfill for improving 
the different parameters of the design process. The different conditions have been numbered as C. \#, where \# represents the number of the condition. This will be useful in next section, where an example will be presented.

In addition, Table 21 summarizes the conditions contained in Table 20 for the situation where the improvement functions are maximized: $N \gg M$. However, as it was observed during the previous comparisons, when the costs or the time spent by FO relative to $\mathrm{AO}$ are big enough to go beyond the threshold values, the preliminary decision-making tool does not improve the design process and it can even worsen it. In addition, if the costs and time invested by SO are of the order of or bigger than AO's ones and $N \approx M>>1$, then neither the time variance nor the expenses variance is improved by FO and therefore, the design process risk is not improved by FO and it can be damaged. In addition, when it comes to the expected expenses, FO is not able to reduce them (and it can even deteriorate them) if the fixed costs of SFA configuration are big enough compared to SA fixed costs to satisfy $(N-M) / 2<\left(E_{D F}^{S F A}-E_{D F}^{S A}\right) / E_{D V}^{A} T_{D}^{A}$. 


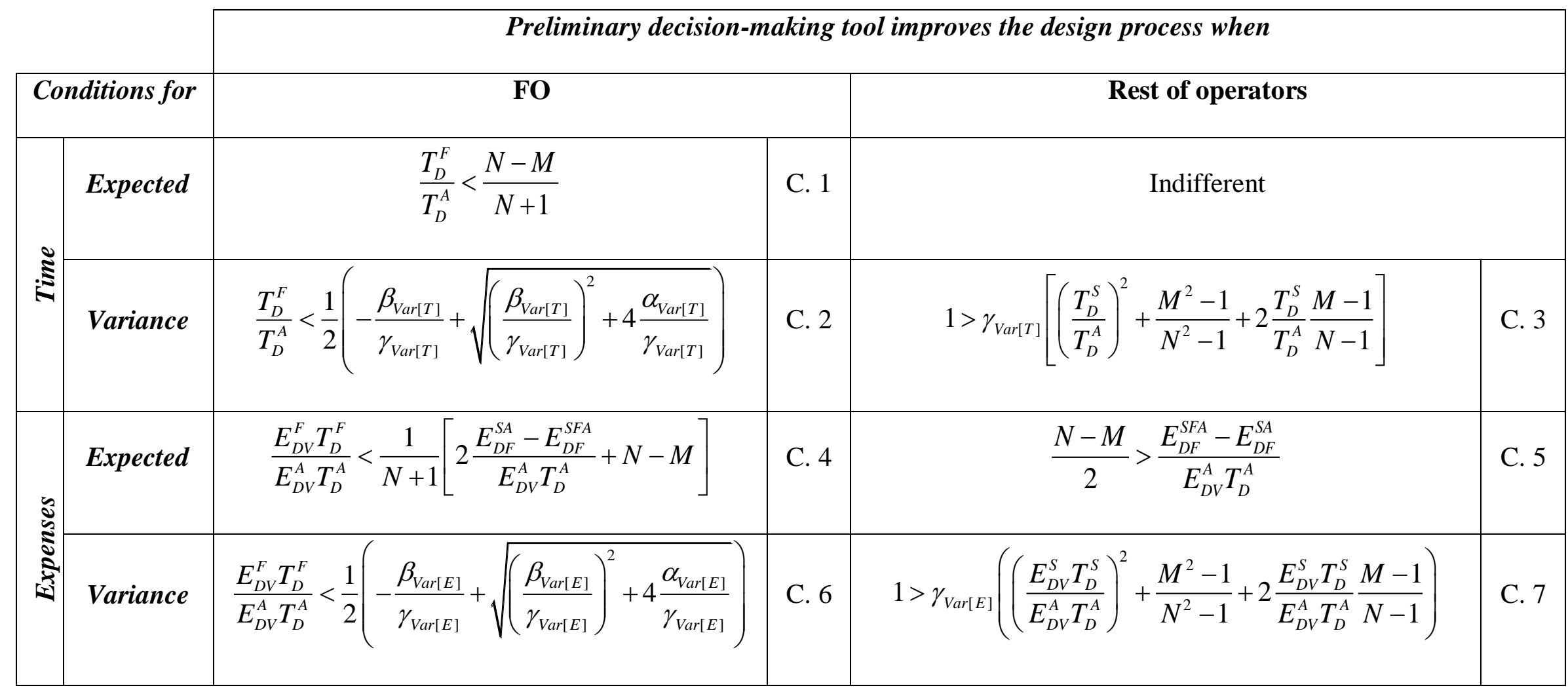

Table 20: Conditions to be satisfied by the preliminary decision-making tool for improving the design process 


\begin{tabular}{|c|c|c|c|}
\hline \multirow{2}{*}{\multicolumn{2}{|c|}{ Conditions for }} & \multicolumn{2}{|c|}{$\begin{array}{c}\text { Maximum improvement case }(N>M) \text { for the } \\
\text { preliminary decision-making tool }\end{array}$} \\
\hline & & FO & Rest of operators \\
\hline \multirow{2}{*}{ 苂 } & Expected & $T_{D}^{F} / T_{D}^{A} \ll<1$ & Indifferent \\
\hline & Variance & $T_{D}^{F} / T_{D}^{A} \ll<1$ & $T_{D}^{S} / T_{D}^{A} \ll<1$ \\
\hline \multirow{2}{*}{ 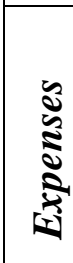 } & Expected & $\frac{E_{D V}^{F} T_{D}^{F}}{E_{D V}^{A} T_{D}^{A}}<<1+2 \frac{E_{D F}^{S A}-E_{D F}^{S F A}}{E_{D V}^{A} T_{D}^{A} N}$ & $\frac{N}{2}>\frac{E_{D F}^{S F A}-E_{D F}^{S A}}{E_{D V}^{A} T_{D}^{A}}$ \\
\hline & Variance & $E_{D V}^{F} T_{D}^{F} \ll<E_{D V}^{A} T_{D}^{A}$ & $E_{D V}^{S} T_{D}^{S} \ll<E_{D V}^{A} T_{D}^{A}$ \\
\hline
\end{tabular}

Table 21: Conditions to be satisfied by the preliminary decision-making tool for maximizing the improvement of the design process 


\subsubsection{Numerical application}

In order to make a numerical application of the previous results, suppose a design process with the following parameters:

\begin{tabular}{|c|c|c|c|c|}
\hline$N$ & $T_{D}^{S} / T_{D}^{A}$ & $E_{D F}^{S F A} / E_{D V}^{A} T_{D}^{A}$ & $\left(E_{D F}^{S F A}-E_{D F}^{S A}\right) / E_{D V}^{A} T_{D}^{A}$ & $E_{D V}^{S} T_{D}^{S} / E_{D V}^{A} T_{D}^{A}$ \\
\hline 100 & 0.1 & 2 & 1 & 0.1 \\
\hline
\end{tabular}

Table 22: Design process parameters example

Two different preliminary tools are included in the design process with the parameters described in Table 23. The first one is a tool such that $t=e=0.1$, while the second one has $t=e=0.9$. The improvement attained with these tools is as follows:

\begin{tabular}{|c|c|c||c|c|c|c||c|c|c|c|}
\cline { 2 - 10 } \\
\hline$M$ & $t$ & $e$ & $\eta_{\mathrm{E}[T]}$ & $\eta_{\operatorname{Var}[T]}$ & $\eta_{\mathrm{E}[\mathrm{E}]}$ & $\eta_{\operatorname{Var}[E]}$ & $\eta_{\mathrm{E}[T]}$ & $\eta_{\operatorname{Var}[T]}$ & $\eta_{\mathrm{E}[\mathrm{E}]}$ & $\eta_{\text {Var }[E]}$ \\
\hline 4 & 0.955 & 0.960 & -0.004 & $\mathbf{0 . 0 0 6}$ & -0.026 & -0.003 & C. 1 & - & C. 4 & C. 6 \\
\hline 10 & 0.900 & 0.700 & -0.008 & -0.005 & $\mathbf{0 . 1 5 3}$ & $\mathbf{0 . 3 2 9}$ & C. 1 & C. 2 & - & - \\
\hline 90 & 0.090 & 0.080 & $\mathbf{0 . 0 0 8}$ & -0.001 & -0.001 & $\mathbf{0 . 0 1 7}$ & - & C. 2 & C. 4 & - \\
\hline 10 & 0.100 & 0.100 & $\mathbf{0 . 7 1 9}$ & $\mathbf{0 . 9 2 7}$ & $\mathbf{0 . 6 8 9}$ & $\mathbf{0 . 9 2 7}$ & - & - & - & - \\
\hline 10 & 0.900 & 0.900 & -0.008 & -0.005 & -0.026 & -0.005 & C. 1 & C. 2 & C. 4 & C. 6 \\
\hline 90 & 0.100 & 0.100 & -0.001 & -0.020 & -0.019 & -0.020 & C. 1 & C. 2 & C. 4 & C. 6 \\
\hline 90 & 0.900 & 0.900 & -0.728 & -2.042 & -0.733 & -2.042 & C. 1 & C. 2 & C. 4 & C. 6 \\
\hline
\end{tabular}

Table 23: Example of different preliminary decision-making tools 
In Table 23 negative values indicate the preliminary decision-making tool worsens the design process. This deterioration is due to the violation of one of the conditions of Table 20 as specified in Table 23 .

It can be observed that when $t=e=0.1$ and $M=10$, the decision-making tool improves the design process in terms of the expected values and the corresponding variances of both resources. When $t=e=0.9$, the decision-making tool worsens the expected values and the variances of both resources.

This numerical application shows how important is to have a very selective $(N \gg M)$ and a very inexpensive $(t \ll 1$ and $e r<1)$ FO. It is an unexpected result that FO produces in several of the previous scenarios a higher improvement in the reduction of the risk associated to the design process than in the reduction of the expected consumption of resources. For example, a $10 \%$ selective filter has a theoretical potential for reducing the monetary and temporal risks by $92.7 \%$.

\subsubsection{Preliminary decision-making tool properties}

As it was explained in the comparison in Section 4.5.5, a preliminary decision-making tool such that $N \gg M$ and $t, e \rightarrow 0$, maximizes the improvement of the design process. Hence, it is convenient to incorporate preliminary decision-making tools which fulfil certain conditions, what can be accomplished by a preliminary decision-making tool that exhibits the following characteristics:

- Compatible: The interpretation of the knowledge by FO has to be compatible with the interpretation of SO and $\mathrm{AO}$ as it was shown in Property 18.

- Absolute: In order to know whether an $\mathrm{AvO}$ is the $\mathrm{BO}$ or not, it does not have to compare it to other solutions as it was explained in Section 4.2.3. 
- Inexpensive: A tool is considered inexpensive when the cost of its implementation (i.e., $E_{D F}^{S F A}$ ) is low, and the costs in every iteration are also low relative to AO's (i.e., $e=E_{D V}^{F} T_{D}^{F} / E_{D V}^{A} T_{D}^{A}$ is small). As it was shown in Sections 4.5.5.3 and 4.5.5.4, this characteristic improves the design process.

- Fast: A tool is considered fast when the time consumed in every iteration is small compared to AO's (i.e., $t=T_{D}^{F} / T_{D}^{A}$ is small). This characteristic improves the design process as it was shown in Sections 4.5.5.1 and 4.5.5.2.

- Reusable: A reusable tool can be applied effectively to the design process of different products, services or processes. Therefore, the fixed costs derived from incorporating a new preliminary decision-making tool for every new product to be designed are avoided and hence, $E_{F}^{S F A}$ can be kept small as required by the discussion held in Section 4.5.5.3 (also contained in Table 20).

- Very selective: As it has been explained along Section 4.5.5, the improvement functions are maximized when $N>>$. A very selective preliminary decision-making tool is able to discard many non-BOs reducing in this way the resources that $\mathrm{AO}$ has to invest evaluating AvOs which are going to be rejected. This leads to the desired relation of $N>M$.

López-Mesa and Bylund (2011) and Benavides (2012) present some concept selection methods that could be considered as preliminary decision-making tools. Next section introduces the concept of design value and focuses on two preliminary decision-making tools; the methodology presented in Section 3 and Axiomatic Design. 


\subsubsection{Design value}

As shown previously, the preliminary decision-making tools which can improve the design process have the following characteristics: compatible, absolute, inexpensive, fast, reusable and very selective. If they fulfill these conditions, then the tool can not only reduce the amount of resources consumed (i.e., money and time) but also their associated risk.

Indeed, the resources consumed during the design process and the associated risk can be linked to the concept of design process value in such a way that when the former increase, the latter decreases. In this way, the overall design value can be divided into the one of the design process and the one of the output. This reflection implies that a valuable output with a very deficient process can render the whole design valueless. In this study we are going to assume that, for a fixed output, the process increases the value when

1. the time and the money required by the process decreases.

2. the variance or risk associated to the consumption of time and money decreases.

3. the acquired complexity is reduced.

Based on this, the value provided by the methodology presented in Section 3 and Axiomatic Design are going to be studied.

\subsubsection{Axiomatic design}

For Axiomatic Design (N. P. Suh 1990), the BO has to fulfil two axioms: the Independence Axiom, which requires the $\mathrm{BO}$ to keep the independence of the Functional Requirements, and the Information Axiom, which requires the $\mathrm{BO}$ to minimize the information content of the output.

The evaluation of whether Axiomatic Design satisfies the previous properties is as follows:

- Compatible: Although, it is an open question whether there exists an industrial counterexample where the axioms do not lead to the BO, the study presented in Section 2 
shows that there exists a theoretical scenario where the Axiomatic Design is not a sufficient condition for robustness and hence for obtaining the BO. Thus, it is a result of this study that Axiomatic Design is not compatible at least in the scenario studied in this thesis.

- Absolute: Theorems and corollaries such as the 'linear designs are better than the nonlinear ones' (Benavides, 2012, which is also obtained in Section 2) or 'symmetric designs are better than non-symmetric ones' (N. P. Suh, 1990) can be used in an absolute way. For example, FO would reject a solution without comparing it to the others if it is not linear or it is a solution without any symmetry. However, the minimization of the information content can be understood as a relative comparison if this minimum is not known a priori. In the Axiomatic Design case, this minimum is equal to zero.

- Inexpensive and fast: The process of FO under Axiomatic Design is simple because the two axioms are independent and can be applied directly on the output. The way how the filter proceeds is to firstly evaluate whether the solution satisfies the Independence Axiom. If so, then the filter should assess if it also satisfies the Information Axiom before sending this solution to the detailed analysis stage. In principle, FO does not need to resort to prototypes, complex simulations or tests to evaluate if an output fulfils these two axioms in a qualitative way. For example it can do it by checking whether the theorems and corollaries are satisfied or not. However, during the detailed analysis stage, expensive simulations and tests (in terms of money and time) may be required to finally assess whether the solution is the $\mathrm{BO}$ or not. Thus, it is plausible that the parameters $t$ and $e$ will be very small if Axiomatic Design is used as the preliminary decision-making tool.

- Reusable: The two axioms are very general (e.g., they do not contain any specific concepts related to a given sector or to other factors which may change the applicability from case 
to case) and they can be applied to any kind of design processes and outputs. The universality of their concepts ensures its use in multidisciplinary teams without extra cost.

- Very selective: The number of initial AvOs generated by a synthesis process is usually very high. However, the key part is to check if the filter conditions of Axiomatic Design are restrictive enough to satisfy $N \gg M$. This is an open topic which should be addressed in future research works. However, some problems already solved lead to infer that this is the case. For instance, Benavides $(2011,2012)$ give an example about the conceptual design of the bearings of a three-shaft turbofan where the Axiomatic Design filter has a value of $M=1$ from a total value of $N=256$.

\subsubsection{Methodology for minimizing dependences}

The criteria presented in Section 3 can work as a filter in the preliminary phases of a design process in such a way that it would only let pass the solutions which have the lowest and weakest number of dependences and hence, it acts as a filter that screens out potential solutions during the design process.

When these main activities are properly executed, they can generate value in two ways as explained previously: firstly due to the output and secondly due to the process. The value of the output is obtained thanks to the minimization of all the dependences (Section 2.6.12), whereas the value of the process can be increased reducing the amount of money, time, associated risks and acquired complexity during the process. These aspects are ensured with activities which have the following characteristics explained previously:

(1) Compatible: The compatibility is established by the objective of Designers. If their objective is to minimize dependences, then these activities are fully compatible. If the 
objective is different, a further study should be performed in order to verify the suitability of these activities in other scenarios.

(2) Absolute: Stimuli-reaction dependences have a minimum which has to be a clear objective. In this regard, this tool can be considered as absolute. However, the minimization of the Internal Costs, can be understood as a relative statement, rather than an absolute one. The reason is that, in order to select which output is the best, designers will likely have to compare one with the others for verifying which output has the lowest Internal Costs. However, this can be solved by setting a lower threshold in such a way that, if the output is below this threshold, it is accepted. In this way, the absolute characteristic can be obtained at the expenses of not reaching the global minimum.

(3) Fast: The working process of the preliminary decision-making tool (or FO) based on the dependences minimization, can be applied without resorting to prototypes, complex simulations and tests to evaluate whether dependences are minimized or not. However, during the detailed analysis stage, expensive simulations and tests (in terms of money and time) may be required to finally assess whether the solution is the best one. Thus, it is plausible that the ratio between how long it takes a preliminary decision-making tool to analyse a solution and how long it takes a full-analysis process to do the same, will be very small if this tool is used as the preliminary decision-making tool. This is relevant since this critical parameter, which has to be as small as possible, is very important for ensuring an increment in the speed of the process and a reduction in its associated uncertainty. Hence, it can be considered that these activities fulfil this characteristic. 
(4) Inexpensive: The previous point makes also plausible that the ratio between how much money it costs to analyse a solution with the preliminary decision making and how much it costs to do the same with a full-analysis process will be very small. This is again a critical parameter, which has to be as small as possible for ensuring a decrease in the design process cost and a reduction in its associated uncertainty. Hence, this tool can be considered as inexpensive.

(5) Reusable: This tool can be used for any product, service or process since it does not contain any specific elements related to a certain industry or field that may change the applicability from case to case. Hence, it can be used in multidisciplinary teams as long as the compatibility characteristic is fulfilled.

(6) Very selective: Previous section explains that there are evidences which lead to think that Axiomatic Design is a very selective tool. Taking into account that the approach of minimizing dependences is more selective than Axiomatic Design as it was proved in Section 2.8, this lets us infer that the evidences for this potential preliminary decision-making tool are even higher.

The previous points, which are not completely conclusive, have shown that the methodology presented in Section 5 has the potential to fulfil all the conditions required to improve the value of the design process except the absolute condition. This condition is important as explained previously because it prevents the design process from having to evaluate all solutions before deciding which one is the best.

A way how this issue could be solved is by setting a threshold on the Internal Costs in such a way that, if they are under a certain value, the solution is accepted. This would make the selection based on Internal Costs absolute and with it, the whole tool. 
It is interesting to note as well how, in principle, Axiomatic Design satisfies the absolute characteristic whereas the methodology presented in Section 3 for minimizing dependences does not satisfy it. The reason behind this behaviour is the different way how they minimize dependences. Axiomatic Design is a sufficient (but not necessary) condition for robustness which only minimizes the number of dependences and it does it in an absolute way. The methodology presented in Section 3 also minimizes the number of dependences and, in this task, this methodology is absolute as well. However, for ensuring robustness, the methodology of Section 3 also minimizes the strength of dependences. This is the task which converts this methodology into a relative one. At the same time, this task is prime for making this tool necessary and sufficient for robustness instead of just sufficient. Hence, the methodology for reducing dependences presented in Section 3 ensures a valuable output but it does so at the expenses of not completely satisfying the absolute condition and hence, not providing as much value to the process as it could provide if it were completely satisfied.

\subsection{Limitations of the proposed approach}

This section summarizes the limitations of the study already presented in the previous lines.

The first limitation regarding the IDP is the selection of the rule-based approach (characterized by a deterministic outcome) instead of the stochastic one. Rule-based processes do not have to represent all existing design methodologies in the industry. Where it fails to represent these existing methodologies, the mathematical model developed in this thesis, resting mainly on this rule-based concept, should not be applied directly. In the same way, the conclusions obtained from it may also differ. However, the reason for having selected this approach is because in this way, as it was explained in Section 4.2.2, this study can demonstrate how a design process which arrives at the $\mathrm{BO}$ does not have to be the best one. 
The second limitation is the imposition on the IDP of the full access to all the useful knowledge. In the real world, the design process will usually have access to just a subset of this knowledge due to the unlikelihood of being able to gather all the useful one present in the world. In fact, it may also happen that, having already all the useful knowledge available, the design process does not know it due to the lack of means for ascertaining the inexistence of any other piece of useful information, which could be hidden anywhere. For that reason, in the real world it is really difficult to ensure the full access to the whole useful knowledge. However, this is not a drawback for the objective of this thesis: the characterization of the ideal processes (not a real processes) for comparing different arrangements of these processes. In this respect, the inclusion of the useful knowledge arises as a direct consequence of this approach.

Regarding the useful knowledge, there is another limitation of this study: the characteristic of stationary (Property 14). This concept, again, arises as a consequence of the rule-based approach despite in the real world this useful knowledge usually increases during the design process (Cross, Christiaans and Dorst [1996]). This would cause a modification in the BO from time to time but, as explained previously, this possibility is removed because, otherwise, it would have averted the comparison presented in Section 4.5.5.

Another interesting point is the definition of the BO. As it has been explained previously, instead of the definition provided in Definition 20, it could have been defined using, for example, utility functions. However, since this study is not focused on how the BO is defined, but on how the IDP differs from the BDP, the only important aspect for our discussion is that the BO exists and it is well defined.

One additional limitation in the models is the evaluation in absolute terms of AvOs by both $\mathrm{AO}$ and $\mathrm{FO}$ : they have to decide whether a given $\mathrm{AvO}$ is the $\mathrm{BO}$ or not without comparing it to 
the rest of the AvOs. Given that usually the value of $N$ is very big, the best situation is an evaluation only based on absolute characteristics in order to locate the $\mathrm{BO}$ as soon as it shows up and, in this way, reduce the consumption of extra resources. In the real world this is seldom the situation, since there are often requirements containing words like "minimization" or "maximization" which could lead to the requirement of comparing different AvOs. However, as mentioned previously, the objective of this thesis is not to study a real process, mainly related to optimization, but to characterize an ideal process, mostly related to conceptual design.

Another remarkable aspect, as explained in Section 4.5.3, is the assumption of a constant value for the resources consumed by the operators in each interaction, what can lead to a loss of accuracy in the results. However, this is not a relevant point for the result of the comparison. In addition, the money invested in the design process is evaluated based on the Net Income, as explained in Section 4.3.1. Instead of Net Income, other measures could have been employed, like cash flow, but it is likely that, in case these other measures would have been employed, the results of this study would not have changed significantly.

A final limitation appears in the fact that the design process configurations only process one $\mathrm{AvO}$ per iteration. In the real world, sometimes many different solutions are selected by the synthesis process and/or analyzed by the analysis process at the same time. However, this feature is not implemented in this study in order to obtain comparable processes.

\subsection{Conclusions}

This section of the thesis has characterized the design process as a process in which the useful knowledge is interpreted in different ways, leading this to the different parts of the design process. Firstly, the synthesis process performs an incomplete interpretation of the knowledge, generating in this way a set of possible solutions. Secondly, the analysis process evaluates these 
possible solutions employing the criteria obtained from a complete interpretation of the useful knowledge and thanks to it, determining the Best Output.

This section of the study has also modelled the Ideal Design Process as a rule-based process which always arrives at the Best Output. As a consequence of this definition, the stationary nature of the useful knowledge arises, what permits the assessment of the impact of preliminary decisionmaking tools on the consumption of resources and its uncertainty and variability during the conceptual design.

The first conclusion is that a preliminary decision-making tool which reduces/increases the consumption of resources (i.e., its acquired complexity) can also reduce/increase its variance, and hence, uncertainty and variability. With them, the risk of the design process is also reduced/increased. Thus, when the consumption of the resources is incorporated, an Ideal Design Process which arrives at the Best Output does not have to be the most appropriate one but there can be others which consume less resources for obtaining the same result. In the real world this means that a design process needs not only to find a good solution, but also to minimize the consumption of resources and its variability during this process. In this task, preliminary decisionmaking tools can play an important role improving the design process. For example, the time consumed in the design process and the risk associated with this time ameliorate when 1) the number of Available Outputs is significantly higher than the number of them that satisfy the preliminary decision-making tool criteria, 2) the time consumed by the preliminary decisionmaking tool in every iteration is considerably smaller than the analysis' one and 3) the time consumed by the synthesis process in every iteration is significantly smaller than the one of the analysis. 
However, there are other situations where a preliminary decision-making tool deteriorates the design process. For example, when the money and the time invested by the synthesis process are of the order of or higher than the analysis' ones and the number of Available Outputs is of the order of the number of them that satisfy the preliminary decision-making tool criteria (both being considerably higher than one), neither the time variance nor the expenses variance is improved by the preliminary decision-making tool and hence, neither does the output risk. Nevertheless, the most critical situation is when the preliminary decision-making tool is not compatible with the analysis process since this worsens the design process for all the values of the different parameters. In this case, the result is catastrophic. For this reason it is really important to determine firstly if a preliminary decision-making tool is compatible.

Finally, if the number of Available Outputs is significantly higher than the number of them which satisfy the preliminary decision-making tool, then this tool maximizes the improvement of the expected value and the variance of the resources consumed. Furthermore, as for the expected consumption of resources, it does not only maximize the improvement, but also makes broader the interval where the improvement can take place.

A final conclusion is that, in order to improve the design process in most of the scenarios, there are a set of characteristics that a preliminary decision-making tool needs to satisfy: compatible, absolute, inexpensive, fast, very selective and reusable. The fulfilment of these characteristics is important for obtaining valuable designs (understood as the conjunction of valuable design process and valuable outputs). It seems quite plausible that the results obtained in Section 2 and 3 have the potential for obtaining valuable designs. 


\section{VALUE PROPOSITION FOR BUSINESS}

\section{APPLICATIONS}

The objective of this section is to discuss how the results obtained along the thesis can impact on business applications. These results can help in the decisions that companies have to make during the design processes, having also the potential for complementing other practices which are common in the industry. This means that these are results which can foster innovation and serve as a tool for gaining competitive advantages over competitors. For this reason, from a managerial point of view, the adoption of these results and the support in the usage of this kind of criteria can have a relevant impact on the results of the company when executed properly.

For example, Section 2 has presented how the objective of a design process in the presence of unpredictable variability and uncertainty should be to fulfill the requirements with a solution that affects and is affected by the minimum number of entities. This will not only ensure robustness but it will also reduce the costs, increase the lifetime and hence, the value of the output. All these elements are nowadays key aspects in almost every industry. This means that during the design processes, organizations should be aware of the elements that are affecting and being affected in the process. The early identification of these elements is an important ingredient in the process of improving the results of the organization.

Section 2 has also shown that designers shall know very well what requirements are mandatory because, if not completely fulfilled, the product is going to be rejected and its value decreased, what reduces company efficiency. Because of this, their accurate identification and treatment shall be prime for organizations. However, organizations not only have to clearly identify them but, if possible, they also have to temper their acceptance conditions as much as possible and, if feasible, remove them. This will be one of the main aspects for obtaining a robust 
design and for this reason, organizations should invest resources interacting with the entities imposing these requirements in order to reach a positive agreement. This, in the end, will facilitate the obtaining of a valuable output. In the same line, designers have to be very careful with the requirements they impose to make the output possible. An inappropriate set of requirements (e.g. too demanding acceptance conditions) can lead to an output with a low value.

An important conclusion of Section 2 has also been the finding that organizations have to be careful with the tools, methodologies or practices they follow during the design process since, even without noticing it, they may be using tools which do not guarantee attaining the most valuable output. This might happen with Axiomatic Design in complex design scenarios where the number of constraints is much larger than the number of functional requirements since, as proved in this work, Axiomatic Design is a necessary but not a sufficient condition for robustness.

Dependences can be difficult to locate and to measure. For this reason, Section 3 has shown how they can be evaluated through costs. An increase or decrease in the cost entails an increase or decrease in dependences and contrariwise. This result highlights a relevant aspect: designing for reducing dependences means designing for reducing costs. Thanks to this, a methodology is proposed and as such, it indicates how an organization can implement in a practical way the results obtained in the previous section to bring about robust and valuable products. This can be an interesting tool not only for $\mathrm{R} \& \mathrm{D}$ but also for improving existing products in competitive markets, where cost and value are key elements. One of the strengths of this tool is that it can be applied to many different industries and in many different kind of designs (not only physical products, but also to non-physical products such as processes and services).

In addition, this study has also shown in Section 4 that not all decision-making tools improve the design process. Indeed, if not selected properly, they can considerably damage it. The 
overarching set of characteristics a design process shall have for enhancing the design process are: compatible, absolute, inexpensive, fast, very selective and reusable. Hence, companies should pay special attention and evaluate thoroughly the kind of decision-making tools they include in their processes for avoiding catastrophic results (for example, the company has already come up with the best solution but it has rejected it due to an incorrect analysis made by an inappropriate decision-making tool).

Section 4 has also presented a model for measuring the consumption and risks associated to different design processes. One important result is that the risk of a temporal or monetary budget is mainly determined by the decision-making tool adopted in the early stages of a design process. If we extrapolate this result to other type of processes, we obtain a valuable receipt for the company: the risk of not accomplishing these budgets during the development of a process can increase if the process is not designed according to the rules obtained in this work. This model can be expanded as needed and it could be very useful in the definition and selection of the most feasible and appropriate design processes depending on the concrete case.

Another important aspect is the scope of the results derived in this work, which apply to: requirement-making, decision-making and result assessment; being all of them key elements in the value-chain of any corporation. As long as the number of dependences is a stable and straightforward indicator, which is also easily implementable in any decision-making process, three extra benefits appear for the company: 1) a new capacity of assessing the degree of innovativeness in a given sector, challenge or need satisfaction, i.e. the degree of maturity of the mainly-adopted solution, 2) a new guide for creativeness in order to obtain more valuable products, and 3) a well-founded company strategy for managing and dosing the value and the benefits derived from any future innovation. Despite the derivations of this work have been obtained with 
a technological product/process in mind, nothing in the proofs or in the argumentations lead us to think that they are not applicable to other kind of outputs such as services, organizations, planning, and management.

Hence, even though theoretical as some of them may seem, this thesis has obtained a set of results whose applicability in the industry is feasible and have the potential of providing the organizations implementing them with a set of interesting advantages. The way how to do it can be the subject of further studies.

In a nutshell, the main benefits for the company cover the following points:

1. Cost savings:
a. Savings in design cost
b. Savings in manufacturing cost
c. Savings in operation cost
d. Savings in maintenance cost

2. Temporal savings
a. Savings in design time
b. Time-to-market reduction

3. Risk reduction
a. Lower probability of temporal budgets breach
b. Lower probability of monetary budgets breach

4. Long-lasting products

5. Comprehensive treatment of Stakeholders requirements

6. Selection of appropriate design tools

7. Higher customer satisfaction 
8. Uncertainty management in early design stages

9. Research and development guidance

10. Company strategy

a. Competitive advantage

b. Product differentiation

c. Establishment of market barriers

11. Unification of the value proposition across the company

12. Detection of sources of value

13. Detection of sinks of value

14. Classification of requirements (into needs and constraints) attending to their addition/subtraction of value

15. Generation of winning designs/solutions

16. Identification of winning designs/solutions

17. Benefits increase (lower cost, higher customer satisfaction, winning designs, long-lasting products...)

18. Increment in the company independence with respect to its environment

19. New tools and indicators for industry status assessment

20. New tools and indicators for benchmarking assessment

21. New messages for marketing strategies

22. Company leadership in the long term

23. Detection of blue/red oceans (Chan Kim and Mauborgne, 2005) 
EARLY MANAGEMENT OF UNCERTAINTY AND VARIABILITY DURING THE DESIGN PROCESS 


\section{CONCLUSIONS}

The objective of this thesis has been to obtain and present new results with the intention of providing designers with tools for dealing with uncertainty and variability in engineering design. This objective has been tackled from different perspectives, showing as well how they are related to each other.

Firstly, a theoretical study has been presented in which a set of sufficient and necessary conditions for robustness has been obtained. The main theoretical result is "minimizing the number and the strength of all dependences as much as possible is a necessary and sufficient condition for robustness". Thanks to this, the Ideal Output is defined as the one with the weakest and the lowest number of dependences. The main difference of this result with respect to other previous design theories is that this new result addressees all dependences instead of focusing only on particular sets of them. The inclusion of all the dependences gives rise to the notion of output value, what permits the classification of dependences into those which generate the output value, called needs, and those which restrict or reduce it, called constraints.

The next step of this thesis has been the conversion of the previous theoretical result into a practical methodology which can be applied by designers in real design scenarios. This presents engineering design from a very different perspective with respect to the previous section. In this case, this practical point of view indicates how to bring ideas to reality from a theoretical field. To this end, a methodology for minimizing dependences during the design process has been presented. A relevant aspect addressed by this part is the fact that dependences can be of a very different nature, what makes it difficult to find a global measure for evaluating them. In order to solve this aspect, a correlation is found between cost and dependences, adopting the former as a measure of the latter. This is put into practice in the methodology minimizing firstly stimuli-reactions 
dependences through the cause-effect matrix and then, the rest of dependences through a cost minimization. Thanks to this, all dependences are minimized and a valuable output can be obtained as a result of the process.

The third section has approached the design process from a different perspective: the evaluation of how preliminary decision-making tools, such as the one previously presented, can affect the design process other than obtaining a certain output. The objective is to show how the same output can be obtained in many different ways depending on the procedures and tools employed in the process and how these elements can render a good output unfeasible when the resources consumed for obtaining it and the risks associated to the process are too high. The first conclusion is that a preliminary decision-making tool which reduces/increases the consumption of resources (i.e., its acquired complexity) can also reduce/increase its variance, and hence, uncertainty and variability. Thus, simply because a design process arrives at the Best Output does not mean that it has to be the most appropriate one. Indeed, there can be others which consume far less resources for obtaining the same result. In the real world this means that a design process needs not only to find a good solution, but also to minimize the consumption of resources and its variability during this process. In this task, preliminary decision-making tools can play an important role if properly chosen and implemented. A result of the work has shown that, in order to improve the design process in most of the scenarios, the preliminary decision-making tool needs to be compatible, absolute, inexpensive, fast, very selective and reusable. The last part of that section has assessed whether the previous characteristics are fulfilled by the design methodology presented in the second section of the thesis. The result is that, even though the absolute characteristic is not completely satisfied (it could be satisfied setting a threshold for the minimization), the rest of the characteristics can be fulfilled, what let us infer that this methodology 
could be useful for reducing the consumption of resources (time and money), uncertainty and the acquired complexity during the design process. In this way, the value provided this methodology can be twofold: the output is valuable and so does the process.

Eventually, the last part of the thesis has discussed how the previous results can be applied in the industry and add value to an organization willing to apply them. As a result of the discussion, the benefits that this work could provide to the company are enumerated, covering aspects such as resources savings, strategy improvement, value increment, risk reduction or benefits increase. However, the detailed description of how these results can be applied is out of the scope of this thesis and they should be the subject of a future study.

Despite the premises and definitions presented in this thesis have led to a generalization of certain concepts, some definitions can still be considered quite restrictive and hence, further research can explore other sets of definitions which could lead to more general results. 
EARLY MANAGEMENT OF UNCERTAINTY AND VARIABILITY DURING THE DESIGN PROCESS 


\section{Appendix 1: Implicit function theorem}

Assume function $\mathbf{D M}_{\mathbf{1}}: \mathbb{R}^{q+r} \rightarrow \mathbb{R}^{r}$ is a continuously differentiable function with coordinates (m, l). Let us take a point such that $\mathbf{m}=\mathbf{m}_{\mathbf{1}} \in \mathbb{R}^{q}, \mathbf{l}=\mathbf{l}_{\mathbf{1}} \in \mathbb{R}^{r}$ and $\mathbf{D M}_{\mathbf{1}}\left(\mathbf{m}_{\mathbf{1}}, \mathbf{l}_{\mathbf{1}}\right)=\mathbf{D M}_{\mathbf{1 , 1}} \in \mathbb{R}^{r}$. If the matrix $\left(\partial \mathbf{D} \mathbf{M}_{1} / \partial l_{i}\right)\left(\mathbf{m}_{1}, \mathbf{l}_{1}\right)$ is regular, then there exists an open set $U$ containing $\mathbf{m}_{1}$, an open set $\mathrm{V}$ containing $\mathbf{l}_{\mathbf{1}}$ and a unique function $\mathbf{f}: U \rightarrow V$, which is continuously differentiable, such that $\{(\mathbf{m}, \mathbf{f}(\mathbf{m})) \mid \mathbf{m} \in U\}=\left\{(\mathbf{m}, \mathbf{l}) \in U \times V \mid \mathbf{D M}_{\mathbf{1}}(\mathbf{m}, \mathbf{l})=\mathbf{D M}_{\mathbf{1}, \mathbf{1}}\right\}$. For the short and long versions of the theorem and their proofs, please refer to Hubbard JH, Hubbard BB (2009). 
EARLY MANAGEMENT OF UNCERTAINTY AND VARIABILITY DURING THE DESIGN PROCESS 


\section{Appendix 2: Projected income statement}

Next points are a summary of the basic aspects of a projected income statement as per the International Accounting Standards Board (2010). All of them should be referred to the life cycle of the product. More information about the projected income statement can be found in Pinson (2007).

- Operating Section:

$\circ$ Revenue (+): Cash inflows or growing of assets.

- Expenses (-): Cash outflows, use of assets or increment of liabilities.

- Cost of Goods Sold (COGS): Cost of the units sold and produced. Production related costs.

- Selling, General and Administrative Expenses: Non-production related costs.

- Selling Expenses: Expenses needed in order to sell the product (salaries of salesmen, deliveries, advertising, trips...).

- General and Administrative Expenses: Expenses needed in order to manage the business (executives' salaries, insurances, office rents...).

- Depreciation/Amortization (-): Allocation of the costs of tangible (depreciation) or intangible (amortization) assets.

- Research and Development Expenses (-): Expenses related to R\&D activities. 
- Non-Operating Section:

O Other revenues or gains (+): Revenues which come from the product's activities that are not the main ones (e.g., income from patents, goodwill). It also includes profits that are either unusual or infrequent (e.g., profit from fixed assets sale).

○ Other expenses or losses (-): Expenses which come from the product's activities that are not the main ones.

○ Finance Costs (-): Costs of borrowing.

- Income Tax Expense (-): Taxes to be paid.

- Net Income: The result of all the previous statements, adding the ones indicated with (+) and subtracting the ones indicated with (-). 


\section{Appendix 3: SA uniform distribution}

The probability of finishing the design process in the step $j$ (event $\mathrm{C}$ ) is the same as the probability of selecting the $\mathrm{BO}$ in the step $j$ (event A) conditioned by the probability of not selecting the $\mathrm{BO}$ in the $j-1$ first solutions chosen by $\mathrm{SO}$ (event $\mathrm{B}$ ).

$$
P(C)=P(B) P(A)
$$

- Event A: Initially there are $N$ AvOs which can be picked up by SO. It is unknown in what order they are going to be selected, however the design process will be finished only once the $\mathrm{BO}$ is selected. In the iteration number $j$, the size of the set of all the AvOs is $\left|\mathbf{C}_{\mathbf{j}}\right|=N-j+1$, so the probability of selecting the BO in the iteration number $j$ is $P(A)=1 /(N-j+1)$

- Event B: The number of solutions which have been selected before the BO is found in the iteration $j$ is $j-1$. Based on this, the number of possible combinations of selecting $j$ - 1 solutions among $N-1$ is

$$
r=\left(\begin{array}{c}
N-1 \\
j-1
\end{array}\right)
$$

However, the possibilities of selecting $j-1$ solutions are

$$
s=\left(\begin{array}{c}
N \\
j-1
\end{array}\right)
$$

Putting together both results, the probability of the event B is

$$
P(B)=r / s=\frac{\left(\begin{array}{c}
N-1 \\
j-1
\end{array}\right)}{\left(\begin{array}{c}
N \\
j-1
\end{array}\right)}
$$


- Event C: Using the results for events A and B

$$
P(J=j)=P(C)=P(B) P(A)=\frac{\left(\begin{array}{c}
N-1 \\
j-1
\end{array}\right)}{\left(\begin{array}{c}
N \\
j-1
\end{array}\right)} \frac{1}{N-j+1}=\frac{1}{N}
$$




\section{References}

Ameri F., Summers J. D., Mocko G. M., Porter M. 2008. “Engineering design complexity: An investigation of methods and measures". Res Eng Design 19:161-179

Andersson P. 1997. “On Robust Design in the Conceptual Design Phase: A Qualitative Approach”. Journal of Engineering Design. Mar97, Vol. 8 Issue 1, p75. 15p.

Andreasen M. M., Hein L. 1987. "Integrated product development”. Institute for Product Development, Technical University of Denmark, Lyngby. Reprint 2000

Asbjørnslett, B. E. and Rausand, M. 1999. "Assess the vulnerability of your production system." Production Planning \& Control, Vol. 10 No. 3, pp. 219-229.

Babuška I. and Silva R. S. 2013. "Dealing with uncertainties in engineering problems using only available data." Computer Methods in Applied Mechanics and Engineering 270 (2014) $57-75$.

Benavides E. M. 2011. "Axiomatic-Design of the main bearing configuration for a several shaft jet engine". 6th International Conference on Axiomatic Design, Daejeon (Korea), 30-31 March 2011

Benavides E. M. 2012. “Advanced Engineering Design: An integrated approach”. Woodhead Publishing, Cambridge/Philadelphia. ISBN 9780857090935

Brown R. L., Gutterman A. S. 2009. "A short course in international business plan”. World Trade Press, California. ISBN: 9781607800026

Cantamessa M., Montagna F. and Messina M. 2013. "Multistakeholder analysis of requirements to design real innovations". International Conference on Engineering Design, ICED13. 19-22 August 2013, Sungkyunkwan University, Seoul, Korea. 
Chalupnik M. J., Wynn D. C. and Clarkson P. J. 2013. "Comparison of ilities for protection against uncertainty in system design.” Journal of Engineering Design, 24:12, 814-829, DOI: $10.1080 / 09544828.2013 .851783$

Chan Kim W. and Mauborgne R. 2005. "Blue Ocean Strategy”. Harvard Business Review Press. US. ISBN: 1-59139-619-0

Chen W. and Lewis K. 1999. "Robust Design Approach for Achieving Flexibility in Multidisciplinary Design.” AIAA Journal. Vol. 37, N08, August 1999.

Chiara M. L. D. 2009. “Uncertainties”. Science and Engineering Ethics (2010) 16:479-487.

Cox G. 2005. "Cox review of creativity in business: Building on the UK's strengths". Independent reviews. Indep Rev. http://webarchive.nationalarchives.gov.uk/+http:/www.hmtreasury.gov.uk/independent reviews/cox review/coxreview index.cfm

Cross N., Christiaans H., Dorst K. 1996. “Analyzing design activity”. Wiley, New York. ISBN: 0471960608 .

Downey K., Parkinson A. and Chase K. 2003. “An introduction to smart assemblies for robust design.” Research in Engineering Design 14 (2013) 236-246.

Durach C. F., Wieland A. and Machuca J. A. D. 2015. “Antecedents and Dimensions of Supply Chain Robustness: A Systematic Literature Review.” International Journal of Physical Distribution \& Logistics Management, Vol. 45 No. 1/2, 2015, pp. 118-137

Dym C. L. 1994. "Engineering design: A synthesis of views". Press Syndicate of the University of Cambridge. ISBN 10: 0521477603

El-Haik B. and Yang K. 1999. "The components of Complexity in Engineering Design.” IIE Transactions. 1999; 31(10):925-934. 
El-Haik B. S. 2005. “Axiomatic Quality: Integrating Axiomatic Design with Six-Sigma, Reliability, and Quality Engineering.” Wiley-Interscience. New Jersey. ISBN: 978-0-471$68273-8$

Eckert C. M., Clarkson P. J. 2010. “Planning development processes for complex products”. Res Eng Design 21:153-171

Engel A., Kenett R., Shachar S. and Reich Y. 2016. “Optimizing system Design Under Degrading Failure Agents". 2016 Second International Symposium on Stochastic Models in Reliability Engineering, Life Science and Operations Management. DOI 10.1109/SMRLO.2016.26

Faneye O. 2005. "Product lifecycle prognosis and modelling in a computer-aided environment." ISBN 10: 3832238611. Shaker, Aachen.

Fowlkes W. Y. and Creveling C. M. 1995. "Engineering Methods for Robust Product Design: Using Taguchi Methods in Technology and Product Development”. Prentice Hall. U.S.A. ISBN-10: 0201633671

Freeman R. E. 2010. “Strategic Management: A Stakeholder Approach.” Cambridge University Press, New York. ISBN: 9780521151740

Goh Y. M., McMahon C. and Booker J. 2007. "Strategies to enhance design analysis reuse: a case study in uncertainty". International Conference On Engineering Design, Iced'07 28 31 August 2007, Cite Des Sciences Et De L'industrie, Paris, France.

Goh Y. M., Newnes L. B., Mileham A. R., McMahon C.A. and Saravi M.E. 2010. 'Uncertainty in Through-Life Costing-Review and Perspectives.” IEEE TRANSACTIONS ON ENGINEERING MANAGEMENT, VOL. 57, NO. 4, NOVEMBER 2010. 
Grayman W. M. 2005. "Incorporating Uncertainty and Variability in Engineering Analysis.” Journal of Water Resources Planning and Management. ASCE / MAY/JUNE 2005. Haik Y., Shahin T. 2011. Engineering design process. 2nd edn. Cengage Learning. ISBN: 0495668141

Hay L., Duffy A. and Whitfield R. I. 2014. "The sustainability cycle and loop: models for a more unified understanding of sustainability". Journal of Environmental Management, 133, 232-257.

Hayter A. 2012. Probability and statistics for engineers and scientists. 4th edn. Brooks/Cole Cengage Learning. ISBN: 1111827044

Hubbard J. H. and Hubbard B. B. 2009. "Vector calculus, linear algebra and differential forms. A unified approach.” Matrix Editions. Fourth edition. ISBN 9780971576650. United States of America

International Accounting Standards Board. 2010. "Presentation of Financial Statements". Accessed 17 July 2010

ISO 9000:2015. 2015. “Quality management systems - Fundamentals and vocabulary”. Praha, Czech Republic: Czech Institute for Standardisation.

Jaynes E. T. 1957. "Information theory and statistical mechanics". Phys Rev 106(4):620-630

Jaynes E. T. 1968. "Prior probabilities”. IEEE Trans Syst Sci Cybern 4(3):227-241

Ji H., Yang M. C., Honda T. 2012. “An approach to the extraction of preference-related information from design team language”. Res Eng Design 23:85-103

Juran J. M. and Gryna F. M. 1988. “Juran's Wuality Control Handbook”. 4th Edition, McGrawHill. U.S.A. ISBN: 0070331766. 
Kalsi M., Hacker K. and Lewis K. 1999. “A Comprehensive Robust Design Approach for Decision Trade-Offs in Complex Systems Design.” Proceedings of DETC'99: 1999 ASME Design Engineering Technical Conferences September 12-15, 1999 - Las Vegas, Nevada.

Kahraman C., Çebı S. 2009. “A new multi-attribute decision making method: Hierarchical fuzzy axiomatic design”. Expert Syst Appl 36 4848-4861

Kanukolanu D., Lewis K. E. and Winer E.H. 2004. “Robust Design of Coupled Sub-Systems using Visualization.” 42nd AIAA Aerospace Sciences Meeting and Exhibit 5-8 January 2004, Reno, Nevada. DOI: 10.2514/6.2004-115.

Kazmer D. and Roser C. 1999. "Evaluation of Product and Process Design Robustness." Research in Engineering Design (1999)1 1:20-30.

Kobayashi H. 2000. "A method of life cycle planning for product eco-improvement." International Journal of Environmentally Conscious Design \& Manufacturing 8(4):27-37

Kobayashi H. 2005. "Strategic evolution of eco-products: a product life cycle planning methodology.” Research in Engineering Design (2005) 16: 1-16.

Kota S. and Chakrabarti A. 2009. "A method for evaluation of product lifecycle alternatives under uncertainty.” International Conference on Engineering Design, ICD’09 24-27 August 2009, Stanford University, Stanford, CA, USA.

Kota S. and Chakrabarti A. 2010. "A method for Estimating the Degree of Uncertainty with respect to Life Cycle Assessment during Design.” Journal of Mechanical Design 132(9), 091007 (Sep 16, 2010). doi:10.1115/1.4002163. 
Kryssanov V. V., Tamaki H., Kitamura S. 2001. “Understanding design fundamentals: How synthesis and analysis drive creativity, resulting emergence". Artif Intell Eng 15(2001):329-342

Kulak O., Cebi S., Kahraman C. 2010. “Applications of axiomatic design principles: a literature review”. Expert Syst Appl 37(2010):6705-6717

Lara-Rapp O. and Benavides E. M. 2015. "Ideal Design Process characterization: the impact of preliminary decision-making tools in the consumption of resources and its uncertainty." Research in Engineering Design. 04/2015; 26(2):97-119

Legardeur J., Boujut J. F., Tiger H. 2010. "Lessons learned from an empirical study of the early design phases of an unfulfilled innovation”. Res Eng Design 21:249-262

López-Mesa B., Bylund N. 2011. "A study of the use of concept selection methods from inside a company”. Res Eng Design 22:7-27

Lu C. Y. S. and Suh N. P. 2009. "Complexity in design of technical systems". CIRP Ann Manuf Technol 58: 157-160

Mader D. P. 2005. “Axiomatic Design and DFSS.” Quality Progress, Aug. 2005, 38,8, ProQuest, p. 77

Matthews P. C. 2011. "Challenges to Bayesian decision support using morphological matrices for design: empirical evidence”. Res Eng Design 22:29-42

Meepetchdee Y. and Shah N. 2007. "Logistical network design with robustness and complexity considerations." International Journal of Physical Distribution \& Logistics Management, Vol. 37 No. 3, pp. 201-222.

Mori T. 2011. "Taguchi Methods: Benefits, Impacts, Mathematics, Statistics, and Applications." ASME Press ISBN 978-0-7918-5969-8 
Morrison S. J. 1957. “The Study of Variability in Engineering Design. Journal of the Royal Statistical Society.” Series C (Applied Statistics), Vol. 6, No. 2(Jun., 1957) pp. 133-138.

Mueller D. and Ganseforth M. M. 2012. "Analysis and modelling of intertemporal relationships in lifecycle design: a case study for investment goods.” Research in Engineering Design (2012) 23: 191-202.

Nedeau J. and Casselman R. M. 2008. "Competitive advantage with new product development: implications for life cycle theory." Journal of Strategic Marketing 16:401-411

Needles B. E., Powers M. 2010. "Financial Accounting (with IFRS)”. $11^{\text {th }}$ edn. ISBN-10: 0-53847601-X

Osborn A. F. 1963. “Applied Imagination. Principles and procedures of creative problemsolving". $3^{\text {rd }}$ edn. Charler's Scribner's Sons, New York.

Osman K., Cebi S. and Kahraman C. 2010. "Applications of axiomatic design principles: A literature review". Expert Systems with Applications. 09/2010; DOI: 10.1016/j.eswa.2010.03.061

Otto K. N., Antonsson E. K. 1993. "The method of imprecision compared to utility theory for design selection problems". ASME 1993 design theory and methodology conference

Padulo M. and Guenov M. D. 2011. "Worst-case robust design optimization under distributional Assumptions.” International Journal For Numerical Methods In Engineering 2011; 88:797-816 DOI: 10.1002/nme.3203

Pahl G., Beitz W., Feldhusen J., Grote K. H. 2007. “Engineering Design. A systematic approach". Springer, $3^{\text {rd }}$ edn. Springer, Berlin. ISBN: 9781846283185.

Peder A. 1997. "On Robust Design in the Conceptual Design Phase: A Qualitative Approach". Journal of Engineering Design. Mar97, Vol. 8 Issue 1, p75. 15p 
Pinson L. 2007. "Keeping the Books. Basic recordkeeping and accounting for the successful small business". 7th edn. ISBN 10: 1419584383

Reich Y. 1995. “A critical review of general design theory”. Res Eng Design 7(1):1-18

Reich Y. and Levy E. 2004. "Managing product design quality under resource constraints". Int J Prod Res 42(13):2555-2572

Shah J. J., Vargas-Hernandez N., Smith S. M. 2003. "Metrics for measuring ideation effectiveness". Des Stud 24(2):111-134

Shaja A. S., Sudhakar K. 2010. "Optimized sequencing of analysis components in multidisciplinary systems". Res Eng Design 21:173-187

Smith K. G., Raines M., Booker J. D. and Batchelor R. 1999. “Allocating Capable Tolerances in Assembly Stack Design.” SAE Technical Paper 1999-01-0053, 1999, doi:10.4271/199901-0053.

Srinivasan R. S., Wood K. L. and McAdams D. A. 1996. "Functional Tolerancing: A Design for Manufacturing Methodology.” Research in Engineering Design (1996) 2:99-115

Stiassnie E. and Shpitalni M. 2007. "Incorporating Lifecycle Considerations in Axiomatic Design.” Annals of the CIRP Vol. 56/1/2007

Suh E. S., de Weck O. L. and Chang D. 2007. "Flexible product platforms: framework and case study.” Research in Engineering Design (2007) 18:67-89

Suh N. P. 1990. "The principles of design". Oxford University Press, Oxford. ISBN 10: 0195043456

Suh N. P. 2001. "Axiomatic design: Advances and applications". Oxford University Press, Oxford. ISBN 10: 0195134664 
Suh N. P. 2005. “Complexity: Theory and applications”. Mit-Pappalardo Ser Mech Eng. ISBN 10: 0195178769

Swift K., Raines M., Booker J. and Batchelor R. 1999. "Allocating Capable Tolerances in Assembly Stack Design," SAE Technical Paper 1999-01-0053, 1999, doi:10.4271/199901-0053.

Taguchi G. 1986. “Introduction to Quality Engineering: Designing Quality into Products and Processes.” Asian Productivity Organization, Tokyo and Unipub. Kraus International, White Plains, NY.

Taguchi G., Elsayed E. A. and Hsiang T. 1989. "Quality Engineering in Production Systems.” New York: McGraw-Hill, ISBN 10: 0070628300.

Taguchi G. 1992. ” Taguchi on Robust Technology Development: Bringing Quality Engineering Upstream.” ASME Press Series on International Advances in Design Productivity. New York.

Umeda Y. and Life Cycle Design Committee of Inverse Manufacturing Forum. 2001. "Toward a life cycle design guideline for inverse manufacturing." Proceedings of the Second International Symposium on Environmentally Conscious Design and Inverse Manufacturing, IEEE, pp 143-148

Vlajic J. V., van der Vorst J. G. A. J. and Haijema R. 2012, “A framework for designing robust food supply chains.” International Journal of Production Economics, Vol. 137 No. 1, pp. $176-189$.

Vose D. 2000. “Risk Analysis - A Quantitative Guide.” Wiley. Chichester, U.K.

Weber R. G. and Sridhar S. C. 1998. “Conceptual design using a synergistically compatible morphological matrix". 1998 FIE Conference. Tempe, AZ, USA 
Westerberg A. W., Subrahmanian E., Reich Y., Konda S., the n-dim group. 1997. "Designing the process design process”. Comput Chem Eng 21(Suppl):S1-S9.

Wieland A. and Wallenburg C. M. 2012. "Dealing with supply chain risks: Linking management practices and strategies to performance.” International Journal of Physical Distribution \& Logistics Management Vol. 42 No. 10, 2012 pp. 887-905

Wood K. L., Antonsson E. K. 1989. “Computations with imprecise parameters in engineering design: Background and theory”. ASME J Mech Transm Autom Des 111:616-625

Wood K. L., Antonsson E. K. and Beck J. L. 1990. "Representing Imprecision in Engineering Design - Comparing Fuzzy and Probability Calculus”. Research in Engineering Design, September 1990, Volume 1, Issue 3, pp 187-203.

Wu Y. and Wu A. 2000. “Taguchi methods for robust design.” ASME ISBN 10: 0791801578

Wynn D. C., Grebici K. and Clarkson P. J. 2011. "Modelling the evolution of uncertainty levels during design”. International Journal on Interactive Design and Manufacturing (2011) 5:187-202. DOI 10.1007/s12008-011-0131-y

Zavbi R., Rihtarsic J. 2009. "Synthesis of elementary product concepts based on knowledge twisting”. Res Eng Design 21:69-85

Ziv-Av A., Reich Y. 2005. "SOS—subjective objective system for generating optimal product concepts”. Des Stud 26(5):509-533

Zolghadri M., Baron C. and Girard P. 2010. "Modelling the mutual dependencies between product architectures and a network of partners." International Journal of Product Development. Vol 10. Nos. 1/2/3. pp.62-86. 
EARLY MANAGEMENT OF UNCERTAINTY AND VARIABILITY DURING THE DESIGN PROCESS 